


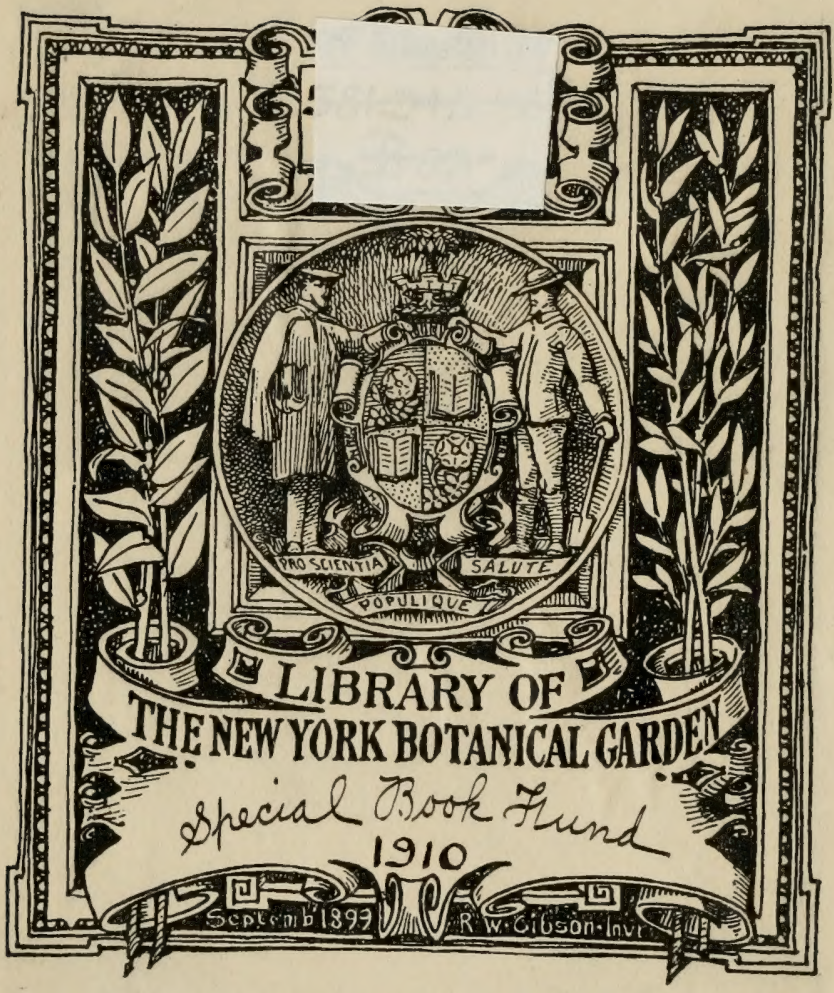




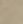

$\therefore$

1

. 




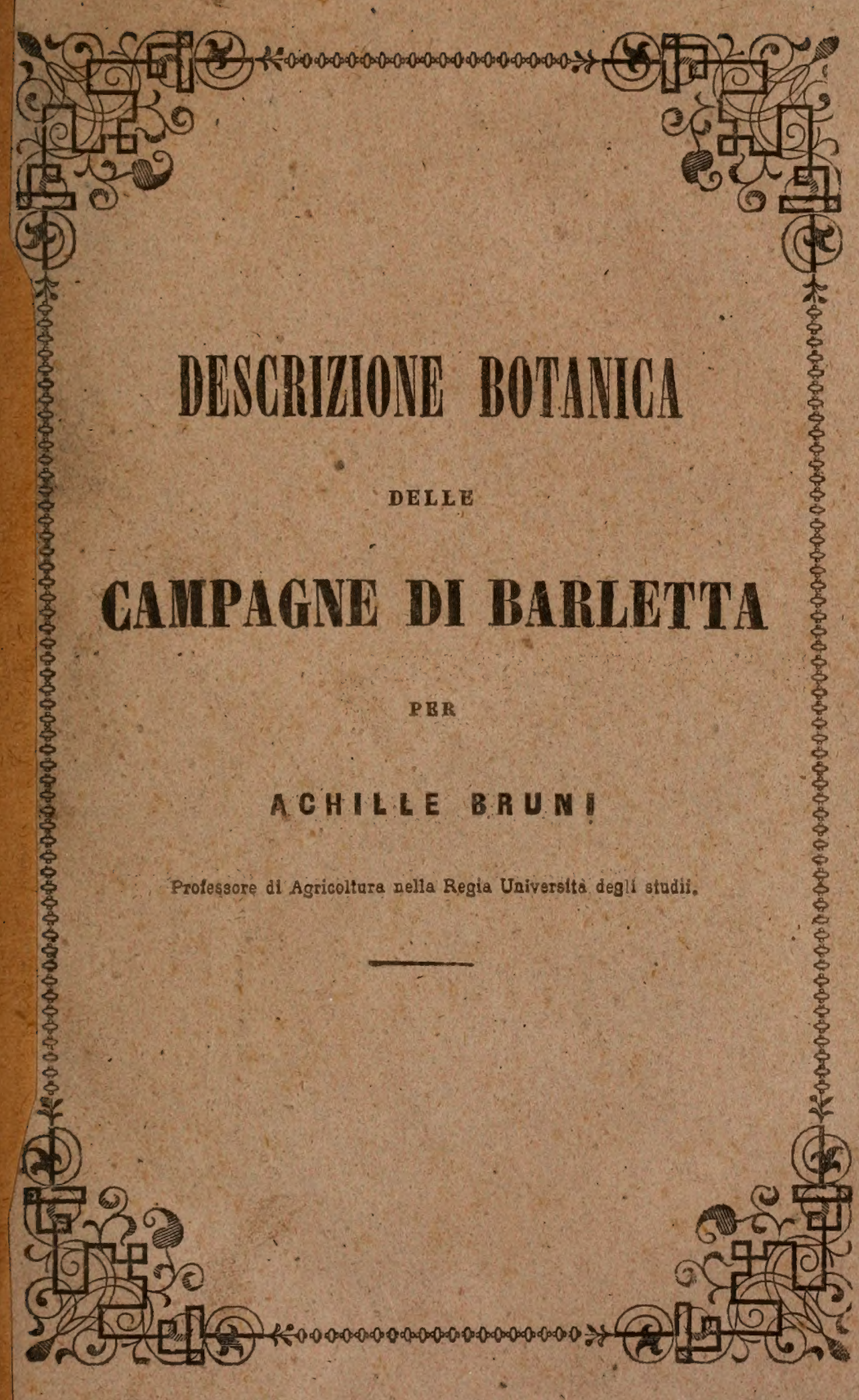




$$
109)^{200}
$$




\section{DESCRIZIONC BOTANICA}

DEL L E

\section{CAIIPAGNE DI BARLETTA}

PER

\section{A CH ILLE BRUN I}

Prolessore di Agricoltura nella Regia Università degli studil.

\section{LIBRARY \\ NEW YORK \\ BOTANICAL, \\ GARDEN}

NAP O L I

STAMPERIA E CARTIERE DEL FIBRENO

Strada Trinità Maggiore $\mathrm{n}^{0} 26$

1857 
.$B 78$ 


\section{DICHIARIZIONE DELL' AUTORE}

\section{LIBR ARY}

NEW YORK

BOTANICAL

OARDEN

Da che nel 1835 mi ebbi l' onore e la fortuna di avere a Maestro di Botanica l' egregio sig. Cavaliere Giovanni Gussone, desio mi punse di tessere un qualsiasi lavoro che avesse in piccolo potuto presentare quel tanto che ne offre la spontanea vegetazione delle campagne di Barletta mia patria. E però nel 1837 l' infausto avvenimento del cholèra avendomi obbligato a ritornare in famiglia per la prima volta, si fu allora che alacremente mossi alla raccolta delle piante in quelle campagne, quantunque l' agitazione d'animo di tutti pel fiero morbo non mi avesse permesso di frequentare le mie piccole peregrinazioni come io il desiderava. Ciò non di meno feci quel tanto che si potè in quelle circostanze, le quali però non furono da tanto da far spegnere in me l' ardente desiderio di visitare il monte Gargàno nella provincia di Foggria; sendochè mi si presentò allora una propizia occasione, e vi andai nel mese di agosto in compagnia di mio Padre che era chiamato in Manfredonia per affari della sua professione chirurgica. Na io in quell' epoca era troppo giovane e appena tirone nella Scienza botanica, perchè avessi potuto far 
tesoro di vegetabili, durante quella gita sul monte. Ad ogni modo raccolsi quel poco che ne permise il tempo e la scarsa o nulla familiarità che in allora colle piante io mi aveva. E mi consolò per altro l' aver saputo rinvenire l'Alyssum deltoideum indicatomi dal mio defunto zio dottor Vincenzo Casale. Egli mi diceva in Barletta queste parole: Fate minute ed esatte ricerche sulle fabbriche vecchie della Chiesa di San Michele, ivi troverete l'Alyssum deltoideum descritto dal sig. Gussone : egli ne desidera $i$ saggi, e voi renderete cosi un servigio al gran Botanico vostro Maestro. Oh! dolci rimembranze de' primi studi!!... Di ritorno in Napoli presentai al sig. Gussone i saggi tutti delle piante da me raccolte nelle campagne di Barletta e altrove, e precisamente sul Gargàno e lungo il lago di Salpi. Egli pieno di piacere per quel presente e con infinita bontà me ne dettava $\mathrm{i}$ corrispondenti nomi, ch' io passava in apposito registro. Fu cosi che si accrebbe in me l' amore per radunare una collezione delle piante barulensi e renderla di pubblica ragione, tanto per un servigio al paese, quanto per offrire un qual siasi lavoretto ai Botanici stranieri che amassero qualche dettaglio di una piccola parte della flora pugliese. Sicchè dal 1837 in poi tutte le volte che mi ebbi il destro di visitare la famiglia, perlustrai sempre l' agro barulense, e ciò mi riusci fare nel 1838-1840 1841-1842-1844-1847. Però, non tutte le contrade furono da me visitate per mancanza di occasioni, e ne restano molte altre le più importanti, quelle cioè al Sud-Est, al Sud, e al Sud-Ovest della Città nei luoghi precisamente che sono destinati alla pastura del bestiame, e che posso- 
no offrire maggior numero di specie positivamente indigene, come più appresso dirò estesamente. Ma se nelle mie istruzioni botaniche mi aveva per guida e lume il sig. Gussone, non minore aiuto mi porgevano le dotte e brillanti lezioni del chiarissimo cavaliere Michele Tenone, dotato da Natura di ampia e nobile facondia, la quale ne invitava con piacere a sentirlo spiegare gli svariati e grandiosi fatti naturali che si osservano nel regno vegetale. Avendo dunque a Maestri due grandi uomini, era per me un ricco patrimonio la somma de'loro ammaestramenti, perchè io mi animassi di più alla carriera (non poche volte interrotta!) dei miei prediletti studi agrari, onde raggiungere qual siasi mèta che la Provvidenza col tempo ne avesse offerta. Sicchè tutte le volte che raccolsi piante nell' agro barulense, fu sempre mia premura di farle prima osservare dal sig. Gussone, sia inviandogli i fascicoli da Barletta a Napoli, sia presentandogliele io stesso, di ritorno nella Capitale. Nel primo caso egli mi onorava di sue lettere accompagnate dalle note dei nomi dei vegetabili con numeri corrispondenti agli altri saggi ch' io serbava presso di me. Nel secondo caso egli mi dettava i no$\mathrm{mi}$, ed io registrava per indi eseguire col tempo il presente lavoro. Nel 1844 lo pubblicai per la prima volta, ma fu ben poca cosa sotto tutti i riguardi. Il prelodato sig. Gussone spesso si riteneva molti saggi di piante, sia per la località, da servire nel suo pregiatissimo e rinomato erbario, sia per inviarne taluni agli altri Botanici stranieri, tra i quali il sig. Heconeicu. Una porzione delle piante spontanee di Barletta fu da me donata all' Imperiale e Real 
Museo di Storia Naturale di Firenze nel 1845, siccome risulta da lettere di ricevuta inviatemi dal sig. cavaliere Axtinoni e dal sig. professor Parlatone. L'autorità classica botanica in Europa del sig. Gussone è conosciuta da lungo tempo, ed io son certo che il nome suo è sufficientissimo per assicurare i miei lettori, e particolarmente i Botanici stranieri (i quali hanno l'amabilità di legger tutto e non disprezzar mai cosa alcuna), che tutte le piante ordinate da me nel presente lavoro, sono precisamente quelle che vegetano nelle campagne di Barletta. Io dunque forte di un' autorità scientifica tanto stimata dall' universale, mi accingo lieto e risoluto ad offrire a'Botanici quel poco che ho potuto raccogliere nel regno di Flora. Laonde, senza più dilungarmi, accenno il piano del presente lavoro, per indi entrare in materia. In primo luogo darò uno sguardo alla posizione topografica delle campagne di Barletta, esponendo in breve quel che ne offre la vegetazione coltivata e quella spontanea. Toccherò leggermente la natura de' terreni superficiali e del sottosuolo. In seguito tratterò di poche cose spettanti al clima del paese. E finalmente esporrò l'elenco delle piante disposte in famiglie naturali per ordine alfabetico: il quale elenco sarà seguito dai nomi volgari del paese che mi riuscì raccogliere per taluni vegetabili, e da varii specchielti contenenti le proporzioni tra le famiglie, i generi, e le specie, in rapporto alla Geografia botanica; oltre le note per un buon numero di piante che mi sono sembrate degne di maggiore osservazione. Un piccolo quadro così tracciato, e che in se racchiude il più interessante di tutto ciò che in quelle campagne influisce 
sulla vegetazione in massa, sembrami che sia indispensabile per questo opuscolo, e che analogo sia un tantino a quanto dice doltamente il celebre De Candolte, figlio, nella sua Geografia botanica pubblicata nel 1855. Nel volume secondo, trattando in generale de' caratleri di vegetazione, e particolarmente del valor relativo de'suddetti caratteri, il uobile Autore cosi si esprime: « Quand il s’agit " de comprendre et de dépeindre l'ensemble de la végéta" tion d'un pays, je m'altacherai d'abord aux caractères " qui frappent tout le monde et qui forment les grands " traits du tableau. Ces caractères peuvent s'exprimer " quelquefois par des chiffres: alors on fait très bien d'en " profiter; mais ce n'est pas toujours le cas. Les formes " ordinaires du langage, si elles expriment des circonstan“ ces très importantes, me paraissent préférables à des ca" ractères numériques de second ou de troisième ordre. "La division générale du sol en marais, prairies, forêts, " terrains salés, terrains cultivés, etc., me semble la cho" se qui, d'entrée, donne l'aperçu le plus juste de la vé"gétation d'un pays. Ce n'est pas seulement un caractère " physique, c'est aussi pour les forèts et les prairies un " caractère botanique, et des plus importants. Si le degré " de civilisation du pays permet de connaître exactement " la proportion de ces grandes stations, on fera très bien " de la donner sous forme numerique. Dans ce cas, les " chiffres expriment d'une manière exacte el abrégée ce “ qu’il est essentiel de savoir. Après cela, je regarderai " comme important de connaître les espèces les plus com" munes, dans les stations qui occupent le plus de place, 
" et en particulier les espèces ligneuses sociales, c'est-à" dire celles qui constituent exclusivement des forèts. " Dans les pays très cultivés, l'indication des principales " espèces agricoles est d'une importance à peu près éga" le ". Se nel citare questo passo del De Candolle mi si volesse obbiettare che quel dotto uomo parla in grande relativamente alla Geografia botanica della superficie terrestre, io farò osservare che il sublime spirito filosofico e scientifico di quel botanico si può applicare tanto al grande quanto al piccolo: ed inoltre aggiungerò che dalla fusione di molti piccoli lavori ne può sortire uno grande più completo e più perfetto sotto la penna di uno o più dotti Botanici; quindi la necessità de' piccoli lavori siccome lo stesso Botanico Ginevrino lo attesta e lo conferma più volte nella sua pregiata opera. Il nostro benemerito cavaliere Michele Tevone fu il primo ed unico, che ci diede una dotta operetta sulla Geografia botanica del nostro Regno, ed il chiarissimo cavaliere Giovanni Gussone nella sua Flora dell' isola di Ischia (della quale pubblicai un articolo bibliografico sul giornale l'Indicatore nel 1855) traccia qualche cenno sulla proporzione delle diverse famiglie, generi, e specie di piante tra il Regno, la Sicilia, la Gran Bretagna, e Ischia. Altri perchè non seguono il nobile esempio di questi due uomini illustri? Non è che io credessi, essere il mio presente lavoro oggetto di Geografia botanica; solo dico che avendo appreso molto nelle opere di Tenone, di Gussone, di De Candolle, e di altri, ho creduto mio dovere adornarlo di quel poco che ho potuto; sì perchè meglio gradito fosse da' Botanici, si perchè 
mi avveggo della necessità degli studì severi sulla Geografia botanica, senza i quali non solo non possiamo comprendere le vere cause per cui certe date coltivazioni non vanno avanti in Agricoltura; ma non potremo neanche trovare la vera via nel positivo progresso della scienza agraria. Forse altri si opporranno a questa mia opinione; ma io pieno del mio convincimento, son sicuro che col tempo verrả il disinganno delle loro obbiezioni.

$$
\text { I. }^{0}
$$

POSIZJONE TOPOGRAFICA DELLE GAMPAGNE DI BARLETTA.

Chiunque muovendo da Napoli volesse visitare le $\mathrm{Pu}-$ glie, dopo percorsa la immensa pianura del Tavoliere, passata la Città di Cirignola, si avvicina al piccolo fume Ofanto in mezzo a fertili campagne di cereali, orzi, biade, e legumi. Quivi basso gli è il terreno dinanzi, ed ur' aria piuttosto grave si avverte nelle vicinanze del ponte che le due provincie di Foggia e Bari congiunge. Passato il ponte, il terreno si eleva dolcemente, e dopo un miglio circa di cammino, si giunge a Canosa, città antichissima situata su di una collina. Si costeggia la Città, e si prende la via di Barletta sulla sinistra del viaggiatore. Per breve tratto la strada serpeggia fra piccole vallate e basse colline tutte coperte di vigneti e massimamente di olivi superbi ed imponenti, fino a che, salendosi una di esse, si arriva sopra vasta pianura piantata a vigne e frutteti, che fanno continuazione delle campagne canosine. Procedendo innanzi, il 
terreno si eleva un poco di più e si mette piede in mezzo ad estesissimi pascoli, de'quali una sezione fa parte del tenimento di Canosa e l'altra di quello di Barletta. Si è in quei pascoli che si trova il punto il più elevato di quelle pianure. Ivi si gode di un ampio orizzonte e si comincia a scorgere di lontano l'Adriatico. L' aria è piuttosto pura e si respira un po' la vita. Da oltobre a tutto maggio vi si gode verdura, e da giugno in poi tutto è bruciato e deserto, e tranne poche specie della famiglia delle Compositae o di altra famiglia in vegetazione rara e dispersa, non vi sono altri vegetabili che risaltano alquanto all' occhio colla loro copia e natural verdura, se non che la Capparis sicula, il Rubus dalmaticus, il Peganum Harmala, il Pyrus cuncifolia, ed il Thymus capitatus, il quale colle sue numerose piante associate, rappresenta tante piccole oùsis di vegetazione attiva negl' immensi pascoli deserti, denominati ul tratturo: così delto, dal perchè serve al transito delle pecore. Continua la via nello stesso aspetto e nel medesimo livello elevato, e dopo qualehe tratto si scorge Barletta posta sull' Adriatico. L' osservatore filosofo e senza prevenzione si fermerà in quel punto, e riconoscerà Barletta situata in un fossato, avuto riguardo a tutta l'estensione del suolo che da quelle amene piagge, gradatamente scendendo, arriva iusino al mare, per un buon tratto non men di otto miglia. Lettore, facciamo pausa un poco, e poi riprenderemo il cammino. Siete voi l'osservatore: $\mathrm{e}$ bene, percorsa tutta quanta l' estesa zona pratense, voi entrerete in un occano di vigneti bassi (supponete il mese di agosto), i di cui grappoli coperti da pampani, 
luttano continuamente contro l'impeto struggitore del favonio (Sud-Orest), allo stesso modo che durante il verno contro il rigor del freddo, l' uomo lutta avvolto in un mantello. Se il favonio imperversa e dura, guai alle vigne: l'uva e i frutti son perduti. Intersecati sono i vigneti da alberi fruttiferi, che giova qui riportare, non per far volume di carta, ma per analogia allo scopo del presente lavoro. Del fico conosco diecisette varietà quasi tutte buonissime. Del granato se ne contano quattro, l'alappio, il dente di cavallo, l'acre-dolce e l'abate verde o senza granelli. Il granato ha questo di singolare, ch'è una pianta rustica, poichè pruova bene nelle pietre secche calcari e nei luoghi palustri: nel primo caso porta frutti più piccoli, ma saporosi: nel seconde più grossi e con granelli molto succolenti. Il cotogno ne offre due, il cosi detto calalogno ed il mollesco o gentile. Il primo è bislungo, assai grosso, lanuginoso, di colore verde-giallo e di un sapore estremamente aspro; l' alberetto che lo produce ha un portamento piultosto altéro con foglie ampie e di un color verde cupo. L'altro poi è tondo, più piccolo, lanuginoso, di colore giallo paglino, e con polpa meno aspra e più gentile: il suo alberetto è più modesto nel portamento, con foglie piccole, e di un verde alquanto chiaro. Viene il sorbo coltivato in pochi luoghi ove porta abbondante frutto. Il ciriegio, l'albicocco, il pesco allignano bene; però il terzo meno de' due primi, tranne nei bassi fondi fertili, ove le sue frutta sono saporosissime : e tra le varietà di albicocche quella detta ananassata è pregiatissima pel sapore e particolare aroma, da superare l'altra deno- 
minata a tabacchiera che vantar si suole per la sua grandezza: ve $n$ ' ha diverse razze, e delle belle. Del melo vi è la sola varietà estiva che produce pomi pessimi da adoperarsi per cibo ai neri. Ma ragion vorrebbe che si bandisse da quei luoghi, poichè le sue frutta nel colmo della state generano malattie perniciose nei visceri de' fanciulli che ne fanno strage. Le mela d'inverno non allignano, perchè il terreno è caldo, secco, e calcare, sopraffatto da un bruciante clima. Il pero rende assai, e conta molte varietà, tra le quali ve n'ha delle ottime, delle buone, e delle cattive. L' avellano raramente si coltiva, ma porta corrispondente frutto. Il gelso moro ed il bianco vi stanno bene; ed il primo a duecento palmi distante dal mare cresce superbo e ben coltivato con sama potagione. Il noce prospera del pari, e meglio cresce nei terreni profondi. Il nespolo comune da pochi si coltiva, e quell'altro detto nespolo del Giappone fruttifica bene nei giardini. Nel 1841 consegnai in Napoli al figlio del giardiniere della Villa Bonelli, Luigi Canfora, i semi di quest'alberetto, che furono subito affidati al terreno nella detta Villa: nel 1847 ne raccolsi il primo frutto colle mie proprie mani. Ivi il pistacchio vero prospera, e porta molti frutti, de' quali pochi sono fecondi mercè l'influenza del polline che ricevono dal maschio della Pistacia lentiscus. Ad averne dunque tutti i frutti fecondati, è necessario introdurre lo scornabeccó maschio, poichè quello che vi si coltiva è parimenti femmina : e due femmine non generarono mai figliuoli al mondo!! Leggete a questo proposito il mio articolo inserito sul giornale il Lucifero. Il giuggiolo ed il lazzeruolo 
del pari sono collivati : il primo è piultosto spontaneo, il secondo s'innesta sul bianco spino e porta saporoso frutto. Il prugno è magnifico, sì per le poche razze scelte, che pel sapore melato e fragrante. V'ha la razza nera e la bianca: lasciate la prima che non serve, e mangiate la seconda; voi v'inebbrierete di squisitezza e di particolare aroma nel sapore. La prugna bianca detta pappacona a Barletta, la prugna agostinella, la prunella d' Andria, quando sono ben mature e scelte, non avrete più che desiderare a tavola. Questi siffatti alberi fruttiferi sogliono intersecare più o meno gl'infiniti vigneti di Barletta. Ma v' ha di più, o Lettore: vedete voi là di lontano quelle grosse colonne di verdura cupa ed imponente, là in fondo a quelle fertili vallate? Non direste somigliar desse ai picchi di montagne in mezzo all' Oceano? Sono i carrubbi, rappresentati sotto forma cilindrica con una maestria di potagione che non vi è chi la pareggi! Lode perciò ai potatori barlettani. Voi potrete sicuramente riposarvi all' ombra della loro periferia, dal mattino al mezzogiorno, respirando l'aura fresca e salutare del Nord-Nord-Ovest. Ma dall' una in poi si avvicina lo scirocco (Sud-Est); fuggitelo, egli vi apporterebbe malanni. Osservate quei boschetti di olivo? Dalla loro piantagione fino a quindici anni vissero in comunità colle viti (dico in comunilà e non associati, perchè in agricoltura sarebbe errore, non essendo prudenza far crescere la vite sull' olivo come si fa coll'olmo e col pioppo ), le quali invecchiate, lasciarono solo gli alberi di Minerva. Sọn diverse le varietà di olivo che vi si coltivano, per olio e per mangiare. Vi è quella delta di Sant' Agostino, e la 
ragusèa, buonissime per tavola. La varietà tulta bianca a cannellino è rara. Dicesi che il suo olio non è buono. Quei filari di alberi e foglic rare e strette costano di mandorli, i di cui frutti mangiati freschi, sono piacevoli, e nei mercati della Cillà se ne fa gran consumo nel mese di Luglio. Se poteste osservarli in Gennajo, Febbraro, e Marzo al tempo della fioritura, ne sareste sorpreso. La infinità de' loro fiori bianchi e bianco-rosei fa un contrasto il più rilevante colla vivace verdura de' sottoposti seminati di cereali e di biade. L'olezzo de' fiori v'inebbria verso le otto del matlino. La varietà dolce è la più comunemente coltivata : assai rara è quella a frutto amaro. Dopo la fecondazione, quando i frutticini sono ancor teneri, basta una gelata, e tulto è distrutto. L'impetuosità de' venti è del pari sommamente nociva, e ne li fa cadere tulti. Quindi la necessità di piantar mandorli a minore distanza; checchè ne dicano gli ostinati critici. Percorriamo più in là le campagne. Vedete quanti nudi terreni in fra le vigne? Sono i piccoli campi addetti alla seminagione de'cereali, biade, e legumi. Coll' andar degli anni si ripiantano a vigneti. E mentre talune generazioni di collivatori si estinguono, le susseguenti ivi trovano vigneti giovani, e viceversa : dov' erano vigneti vecchi, il grano riprende piede. Ed è così che l' economia agricola in siffatta industria porta tacitamente la palma a favor della scienza agraria. Cioè? L' avvicendamento agrario tra le granaglie e la vite ogni quindici o venti anni. Gli è un bel periodo sul quale nessuno pensa ; e intanto l' arte, l' industria, l' uomo, il tempo, l'interesse, l' esperienza, ed il buon senso : tutti quesli elementi 
uniti insieme, e favoriti dalla stessa natura, eseguono tacitamente ciò che la scienza ne detla e insegna. Il credereste? l' uomo esegue tutto a capello, se a gradi a gradi cammiua. Orni slancio troppo elevato rovescia tutto.!. Voi vedrete da qui a poco un esempio chiarissimo a favore di questo importante argomento, (cioè camminare a gradi in Agricoltura) sulla coltivazione de'poni di terra , comunemente patate. Avanziamo intanto il passo, ed eccoci dolcemente scendere in una ridente vallata assai fertile. Gli è quella di casavccchia cosi detta. La vedete quanto ¿̀ larga? Due, tre in quattro mila palmi? La misureremo in seguilo. Per ora facciam senno sulla sua posizione topografica, e passiamo avanti. Dista dalla Ciltà poco men di tre in quattro miglia. Continuano i vigneti cogli stessi alberi fruttiferi. Ma veh! La Villa Bonelli ne mostra a sinistra. La è ridente e graziosa. Quivi, gli agrumi si coltivano con esattezza mercè le cure dell'abile e attivo giardiniere Gennaro Canfora. Essi vi prosperano, e dànno abbondante frutto; ma le gelate li padroneggiano, sendo che nella massima parte trovansi piantati in sito aperto e molto esposto al Nord. Negli altri giardini privati anche si coltivano, ma non riescono cosi perfetli come più volte ho visto negli altri luoghi della Provincia di Bari. Le ragioni stanno nella natura del terreno sassoso-calcare assai; in quella del clima molto secco; nella scarsezza delle acque dolci; ed in un poco di pigrizia e testardaggine per parte di quei pochi proprietari i quali vorrebbero le frutta degli agrumi, e intanto mai vogliono migliorare lo stato del terreno. Presso l'Ofanto, nei giardini delle masserie, 
gli agrumi anche danno un buon prodotto. Passiamo inoltre. Vedete quei mandorli, fichi, e granati in quantità ? Gli è questa la zona degli ortolani, tutta cinta dall' Atriplex Halymus e dal Lycium europæum, che formano impenetrabili siepi. Coltivano bene, anzichè no. Ottimi broccoli neri, o di Malta che dir volete: ma facilmente imbastardiscono; voluminose barbabietole rosse, e qualche altra verdura. Osservate quegli uomini, che vanno di qua e di là con due recipienti di argilla cotta, ornati di manichi, e corrottamente chiamati ugnuturi? Due recipienti io dico, uno alla mano dritta, l' altro alla sinistra, e pieni di acqua. Servono essi per annaffiare gli ortaggi che ordinariamente soglionsi coltivare in aiuole paralellogramme. Gli uomini si stancano andando avanti e dietro, l' acqua si sciupa, il tempo si perde, la spesa e la fatica si dissipano. E perchè ? Non vogliono introdurre il metodo di annaffiamento per infiltrazione come si esegue presso la città di Bari, nei contorni di Napoli e in altri siti del Regno. Gli è vero che il terreno ivi è argilloso; ma un poco di altività e studio sarebbero sufficienti a modificare ciò ch'è barbarismo e vergogna nell' arte agraria. Basta così : entriamo in Città, percorriamo la strada della Sottointendenza e quella di Santo Andrea, ed eccoci alla marina. Guardate a sinistra e a dritta. Campi assiepati dall' Atriplex Halymus che ama ardentemente l' aria marina. Secondo il Sig. E. Mever questo frutice, o alberetto al dir degli altri, trovasi spontaneo al Capo di Buona Speranza, ove cresce altresi la Salsola fruticosa: indigena presso le foci dell'Ofanto. Veh ! com' è superbo e gigantesco senza col- 
tura. Rende grandi servigi a quei terreni arenosi, perchè sotto forma di folta siepe li difende dal vento Nord. Forse le sue ceneri contengono molta soda! Il celebre chimico malageti non ne parla nella sua pregiatissima chimica agraria. Ne attendiamo le analisi da' Chimici, siano stranieri, siano regnicoli. L' essenziale si è lo studio a beneficio nostro e a benelicio di tulti. Mirate quanto cotone. E una pianta bene adattata in questi luoghi. Nutrimento copioso, acqua sottostante, buona coltivazione, e i contadini se ne trovano contenti. In questi fondi arenosi vicino mare si coltivano il Gossypium siamense, il Gossypium herbaceum, il Solanum tuberosum, il Lycopersicum sativum, il Ricinus communis, il Phascolus vulgaris, qualche specie di Dolichos, e molte scelte razze della famiglia delle Cucurbitacex. L'Allium cœpa è oggetto d'industria e di ricchezza pei contadini, che ne coltivano delle varietà belle, grosse, e gentili: e tutte queste piante formano l'agricoltura estiva. Nel verno, orzo, broccoli, legumi, e piante simili. Raccolti questi vegetabili in primavera, si zappa e si vanga a tre palmi il terreno. Il vangatore e lo zappatore si bagnano i loro piedi nell' acqua copiosa ch' ivi sorge nel coltivare a tanta profondità. Rivoltano il terreno: ed in primavera avanzata tutto è affidato al medesimo che si tien sempre mondo dalle erbe. In autunno si eseguono le raccolte; ed i campi offrono un caos di erbe spontanee floride e gigantesche (per esempio la Digitaria sangruinalis ed il Panicum crusgalli coi culmi lunghi circa tre palmi misurati appositamente), le quali poi si soversciano dopo essere state decimate per foraggio agli animali. 
E tutte siffatte coltivazioni le troverete egualmente lunga il mare, cioè all' Est della Ciltà, e all'Ovest, precisamente ove siamo in mezzo a questi ricchi campi arenosi coltivati a patate, o pomi di terra che dir vogliamo. Quivi riescono le patate superbe e assai gentili, ed $\mathbf{i}$ coloni si arricchiscono. E sapete perchè? Per la quantità e qualità. E tutto ciò ottengono mediante una buona coltivazione che appresero e perfezionarono a gradi a gradi, seguendo la regola che poco fa vi accennai. Se non vi è discaro di seguirmi, io da quì a poco vi farò osservare una lunga estensione di fondi arenosi silicei coltivati allo stesso modo colla massima esattezza per le stesse specie di piante che poc' anzi vi ho citate. Vedete bene, siamo giunti in un punto, ove non possiamo passare più avanti, perchè il mare lo impedisce. Quella barchetta ne attende: noi in essa saliremo, e con una breve traversata, costeggiando le fabbriche di questo piccolo fortile (denominato comunemente paraticchio)(1), giungeremo sull' opposto lido. Ed eccoci finalmente giunti. Osservate; da questo punto, ove siamo sbarcati, sino alle foci dell' Ofanto si contano quattro miglia di cammino. Le coltivazioni sono sempre le stesse, come prima vi ho detto, non escluse le patate. Questi terreni sono denominati comunemente arene, e con ragione, poichè di terre arenose silicee sono formate. Quando io mi contava dodici anni di età, cioè verso 1828 in 1829 , mi ricordo che questi fondi

(1) Se mal non mi avviso, pare che questa voce volgare paraticchio significar volesse nel linguaggio popolare piccola difesa : ed in verili a questo scopo furono innalzate quelle fabbriche. 
erano assai estesi, ed il mare stava molto lontano da questi naturali rilievi di terreno argilloso marnoso, che qui vedete a sinistra, alti circa centocinquanta palmi e più, e che poi a gradi a gradi abbassandosi, spariscono del tutto nelle vicinanze della massaria di Santo Lazzaro. All'opposto poi, all' Est della Città il mare era vicino alle terre argillose seminatoriali, ed $\mathbf{i}$ fondi arenosi di quella contrada erano ristrettissimi e coltivati pure come questi che avete sott' occhio. Presentemente la scena è tutta cambiata, da molti anni a questa parte, e a gradi a gradi. Verso l' Est delia Città il mare si è ritirato, ed i terreni silicei coltivabili si sono distesi immensamente. All' Ovest poi, in questo punto, ove siamo, il mare ha invaso tutto; e mentre prima era coltivato e ricco di prodotti campestri e ortalizii, ora non vedete altro che ammassi di alga marina che il mare deposita agli argini di questi ristretti campicelli che osservate, i quali poi più estesi li vedremo di quì a poco, a misura che ci avvicineremo all' Ofanto. A questo proposito vi piacerà, spero, qui riferirvi poche parole dell'illustre Arciprete grovene, da Molfetta (chè, poi studieremo le sue opere), quando scrisse le notizie geologiche delle due Puglie Peucezia e Daunia tanti e più anni or sono. Egli diceva così : Già è notissima cosa allontanarsi sempre pii il mare da quei lidi per le torbe de'fumi. In tempo della mia fanciullezza le acque del mare battevano il castello di Barletta; ora il mare vi è lontano per una cinquantina di passi. Nel 1851 io osservai che il mare n' era allontanato per centinaja e centinaja di passi. Eccoci intanto bene inoltrati nel cammi- 
no, e avvicinarci a quella specie di laguna, che da quì vedete poco distante da noi. E dessa la così detta fiumara, cioè una grande estensione di quei fondi, parte silicei, e parte melmosi e argillosi, sempre allagati per più mesi dell' annata. Durante il verno le correnti dell'Ofanto inondano le campagne adjacenti; e quando le acque gonfie del fiume s' innalzano in qualsiasi tempo ed escono precipitosamente dal Joro letto, allora guai, guai e lamenti a tutti! E fondi, e terre, e carri, e covoni, e biche, e strade, e animali, e pastori, e massari, tutto tutto è nel pericolo della perdizione. Povere piante e future raccolte! tutte sepolte nell' argilla o nella sabbia. Le acque cosi accavallate inondano estesissimi e lunghi tratti di terreno. I soli alberi si trovano bene, perchè inorgogliscono colI' acqua e col terriccio copioso; ma talvolta anche sono sbarbicati, e trascinati o nelle adjacenze, $o$ al mare. Nel Febbraro 1850 , ahi ! quale sciagura.!. crollo il ponte che congiungeva Barletta alle Reali saline. Opera di parecchi secoli, al dire degli storici e degli eruditi. Non si trovò una pietra di tutto il materiale caduto : tutto era inabissato nella melma e nelle così dette lòpe o voragini Ofantine. Si racconta che poche ore prima di crollare, erano passati parecchi carri carichi di grano. Quale morte orrenda avrebbe colpiti quei poveri che li conducevano, se si fossero trovati sul ponte nel momento del suo crollo?.. Ammiriamo, o Lettore, i tratti della Provvidenza, e facciamo senno!!! Vedete là gli avanzi delle sue fabbriche? Secondo gl'ingegneri di ponti e strade, erano alte sessantaquattro palmi a contare dal livello delle acque. Presente- 
mente in questo stato miserando fanno spavento! Nel verno 1851 in compagnia del giovane Artista Sig. Giambattista Calò, di Barletta, l'osservai da vicino ed inorridii, e ne feci eseguire dallo stesso un piccolo disegno. Guardate e tremate !! Ma noi lasceremo a dritta tanta rovina, e muoveremo un poco alla sinistra, costeggiando le acque del fiume. Salendo dolcemente quelle amene piagge, vedremo qualche cosa di buono. Osservate qui attentamente: il suolo si eleva dal livello delle acque del fume per pochi palmi, e continua cosi alla nostra sinistra ingolfandosi per lungo tratto, ove nirate vigneti floridi e robusti, piantati in una dolce vallata. Gli è questa appunto che fa continuazione con quella che traversammo più innanzi, quando da Canosa muovevamo a Barletta, cioè la vallata di casavecchia. Ebbene siffatto largo e fertile incanalamento di terreno si prolunga da questo punto, Ovest-Sud-Ovest della Città, fino all'Est-SudEst della medesima. Serpeggia per varii modi, e verso la metà della sua lunghezza (al Sud) dista dalla Città per circa tre in quattro miglia. Rinomati sono i terreni di questo serpeggiante avvallamento per la loro fertilità; e soprattutto vi si distinguono varii punti interessanti, quali sono quelli denominati comunemente; fondo di Campanile, Sbomma, Casavecchia, Conca d'oro, Belloluogo, Crocefisso, Callano, Santo Luca. Tutti questi bassi fondi sono tante diramazioni di una medesima posizione topografica di terreno piano e fertile. Essi si comunicano in varii punti, serpegggiando di qua e di là, e formano una zona campestre a sè, cioè la migliore di tutte, tenendo l'intermedio tra le campagne più elevate e sassose (che sono le 
più lontane dalla Cittai), e quelle che offrono una specie di uniformità piana e monotona. In somma tutta la zona fertile de' terreni avvallati si può considerare come la parte centrale di tutto il tenimento barulense; e si estende dall'Est all'Ovest. E che più? Fin là, cioc̀ fino alla contrada di Santo Luca ho perlustrato l' avvallamento, il quale da questo punto, ove siamo, vicino l'Ofanto, fino alle collinette di Santo Luca raggiunge la lunghezza di circa otto miglia e forse più, se mal non mi ricordo. Ma su di ciò prenderemo notizie più precise a miglior tempo. Passiamo avanti, perchè dobbiamo camminare altro lungo tratto, e vedere un po di tulto. Però facciamo un' osservazione interessante pria di riprendere il cammino. E sarebbe la seguente. Chi ne assicura che un tempo fu, in cui le acque dell'Ofanto nella stagione degli straripamenti divergendo alla loro diritta, avessero bagnato o allagato questo incanalamento fino alla contrada di Santo Luca, e che di là poi internandosi in altri terreni, avessero messo capo nelle paludi, traversando così le campagne all' Est della Città? Io intendo parlar di secoli remoti. Ha noi ritoccheremo quest'argomento. Eccoci dunque sopra amene piagge che ne offiono un bel orizzonte. A sinistra, vedete che sterminata estensione di terreni seminaturiali delle masserie. Quelli perfettamente nudi di erbe sono maggesi da seminarsi nell'anno che segue. Degli altri chi offre aurate stoppie e chi quantità di cenere e stoppia carbonizzata coll' abbruciamen10. Questi pascoli che calpestiamo sono le cosi dette mezzane delle masserie. Guardate a drilla che immensa pianura alle falde di queste collinette. L un oceano di rigogliosa e sva- 
riata verdura che offie una specie di panorima. Avvertite quel mormorio là basso? Sono le acque dell' Ofanto che infrangendosi in fra i sassi e ciottoli, si precipitano poi in quelle voragini che i mandriani chiamano lüpe, come più avanti dissi. Guardatene da lontano l' argentea fascia; che grandioso scherzo naturale! E quei giganteschi gruppi di verdura cupa ed imponente? Sono gli olmi (Ulmus suberosa) che vivono associati e slidano tulte le bufere. Li sotto vi è un' ombra cosi densa che il sole non vi può. Se vi penetrate, tetro n'è l' aspetto; ed il cupo silenzio (spesso interrotto dal garrir degli uccelli) che vi regna e la solitudine fanno tale e tanta impressione sull' animo, che destano l' idea dell' esilio in luogo recondito ed inaccessibile. $\mathbf{E d}$ in vero i colossali macchieti di rovo, di bianco spino, di prugno selvaggio , paliuro, vi lacerano e abili e carni. Difficilmente potrete camminarvi senza la guida degli uomini pratici di quei luoghi : intendo dire de' mandriani (comunemente gualani) che ivi menano a pascolare il grosso bestiame. Una grossa vacca difficilmente si distingue in quei terribili cespugli e fruticeti, tanta è la foltezza dei loro rami. Osservate quanti pioppi bianchi sparpagliali e con elevatissima chioma. Ve n' ha degli annosi, due de quali nel 1847 avevano sul tronco di ciascun di essi una circonferenza di tredici palmi a poca distanza dal terreno. $V$ ' ha pure il pioppo nero, ma non è indigeno, è figlio di una antica coltivazione. Vedete quanto sono magnifici quei frassini a chioma spaziosa e florida. Potrebbero servire per l'industria della manna, se non ci fusse un certo disprezzo a tanta provvidenza! A fianco loro crescono due specie di 
salici con una vistosa vegetazione. Quanta Phyllirea, Clematis vitalba, Vitex agnus castus, Olea curopæa oleaster, Tamarix gallica et africana, Pistacia lentiscus. Sono tutte piante che riescono rigogliose e belle. I possidenti prendono cura degli olivastri, poichè ne li svelgono, li trapiantano ne' campi, e poi gli annestano. All' ombra di tanta verdura si può reggere sino a mezzodi, ma nelle ore pomeridiane lo scirocco (Sud-Ovest) si rende molesto in ragion composta dell' elevazione di temperatura che regna lit basso, dell' umidità, e delle esalazioni nocive ch' emanano i circostanti terreni, i quali, lasciati in secco dalle acque del fume, facilitano col calorico del sole la putrescenza delle sostanze organiche, non escluse le miriadi d' insetti morti o presso a morire. A fianco di questi silvestri luogyli si coltivano sterminati campi alla seminagione de' cereali, e propriamente de' grani duri che riescono a meraviglia (in quei terreni argillosi denominati terreni d'ischia), e che hanno tanto nome in commercio. Passiamo avanti, costeggriando l'Ofanto, ed eccoci sulle ridenti colline della famosa Canne. Voi vi avete solt' occhio un orizzonte ameno, e lo stesso teatro che arete visto poco prima. Cioè gruppi di sostenuta e gigantesca verdura negli olmi, frassini, pioppi, salici, rovo, bianco spino, casto agnello, tamerigio, lentisco, ed altro. Se ponete mente a tanti culmi di Arundo plragmites e Arundo donax le di cui pannocchie torreggiando su i macchieti, si agitano ad ogni muover di vento, voi direte, là sotto alcerto l' acqua ristagna per buona parte dell' anno. Guardatevi di por piede in quei terreni; vi affonderete sino a due palmi c più, e re- 
sterete inceppato nell' argilla e nella melma come gli uccelli sulla pania del visco e del loranto. Se voleste continuare da qui sino al ponte di Canosa, trovereste le stesse condizioni fisiche e topografiche, e sparire a gradi a gradi gli olmi colossali, ed i pavoneggianti frassini. Vi sono però ricchissimi erbaggi che formano la vera fortuna di quei proprietarii: Na noi qui facciamo riposo; dissetiamoci a questa bella fontana di Canne, modesta e povera di acque limpide, dolci, e salutari. Raccogliamo il Nasturtium officinale; e dopo, con una lunga traversata a passo avanzato, sorpasseremo la strada nuova di Canosa, che percorremmo prima; e passando a traverso di pascoli, seminatorii, vigneti, oliveti e mandorleti, ci troveremo sulla strada nuova che congiunge Andria a Barletta. Oh! che ameni e ridenti colli!... Avvertite un' altra energia nella respirazione e nella vita? Gli è tulto effetto dell'aria pura e salutare, assai migliore di quella che respirammo sulla via di Canosa. Quella via risente un tantino gl' influssi del vicino Ofanto, e questa no, perchè è lontanissima da acque stagnanti ed è tutta mediterranea. Aggiungete la sua piccola elevazione dal livello del mare, e vi avrete maggior purezza e bontà nell' aria. Io vorrei servirvi a farvi conoscere quanta è precisamente l' elevazione dal livello del mare di questa via e di quella di Canosa; ma nol posso per ora: lo spero in appresso. Quivi nella Primavera tutto è ridente, e l'abbondanza sterminata degli asfodeli ramosi in fiore ne accrescono la bellezza. Vedete là basso quegli olivi ? Il loro portamento è tult' altro di quelli che vedemmo in altre campagne. Sono più ricchi di frasche, più rigogliosi 
e di aspetto più gaio. Sapete perchè ? Le loro radici sono distese ed insinuate nei crepacci della pietra calcare ( comunemente pietra viva) ed ivi trovando più copioso e sostanzioso nutrimento resistono vieppiù agli ardori dell' aere infocato dal sole. Battiamo avanti la via, e traversiamo tulti quei pascoli che ci parano dinanzi. Son dessi continuazione del cosi detto tratturo ad uso delle pecore, come in altri siti v'indicai. Eccoci su di un'altura da dove si vede Barletta sempre in un fossato. Guardate, siamo giunti sulla strada nuova che conduce a Trani. Abljiamo alle spalle quella Ciltà; di fronte ci sta Barlella; a sinistra il tratturo; a dritta l'Adriatico. Ma il mare è ancor distante da noi. Voi ne avvertite il mormorio delle onde col favor del vento; ma tra esso e noi havi quella immensa e bassa pianura coperta di alberi fruttiferi. É la contrada delle paludi. Tutti gli alberi fruttiferi vi pruovano bene, meno il carrubbo e l'olivo. Vedete quei carrubbi là basso mezzo bruciati ed ingialliti? L'aria marina loro è contraria, e sviluppano una vegetazione languida e stentata. Scendiamo un poco nella pianura ed avviciniamoci agl'innumerevoli fossati e canali di acqua, che s'intersecano fra loro in mille modi. Vedete quante piante indigene lungo $\mathrm{i}$ loro argini? La loro vegetazione è oltremodo rigogliosa per la copia del terriccio e delle acque. In questi campi, ricchi prodotti si raccolgono delle uve (che si vendono per uso di tavola con grappoli pesanti più rotola) ed in altre frutta, massime le pere. Gli ortaggi estivi, i laggioli, il ricino, ed il cotone sono le principali industrie de' coltivatori. Ma quella de' poponi, congiunta al- 
l'altra che si fa nelle arene vicino mare, costituisce la vera ricchezza de' contadini. In questi luoghi perniciosa è la dimora prolungata e particolarmente il pernottare. Nelle ore matutine si può stare, ma in quelle serotine è massima imprudenza. Poco prima del tramonto del sole lo scirocco (Sud-Est) incalza bene. Un' aria umida e pesante vi gravila sulla persona: un vento fresco vi batte sul viso, e voi avvertite nelle membra un certo ribrezzo ributtante e nojoso. Se siete riscaldato e sudato, camminate sempre, senza fermarvi; in contrario potreste incorrere in una intermittente, o in una perniciosa. I coloni che frequentano questi luoghi, sono i martiri dello scirocco, e spesso le vittime di ostinate febbri. Coloro che a Barletta ne sono attaccati, subito al tempo della convalescenza ricuperano nella citta di Andria, la di cui aria salutare sta a quella pesante di Barletta come sta l' aria del colle di San Martino presso Napoli a quella fetente del quartiere Porto, Rua catalana, e altri antichi siti bassi e luridi della Metropoli. Nel 1838 anch'io sperimentai il cambiamento di aria in Andria dopo una intermittente sofferta in patria. Bastarono pochi giorni di dimora colà, e dalla morte passai alla vita. Vi ricordate quel punto elevato e salutare che abbiamo toccato sulla via che mena in Andria? Ivi è il primo grado d'aria pura, come si fa a Napoli a godere l'aria dell' Infrascata, e quindi a San Martino. E una gradazione continuata da quel sito sino ad Andria, e da questa Città alle basse montagne che diconsi murgie nell' interno della Provincia di Bari. Ora vi basti questo piccolo quadro delle fertili paludi, ma assai perniciose. Procediamo sullo 
stesso livello basso, ed inoltriamoci in quello avvallamen. to ampio e ferace che ci sta di fronte: traversiamo la strada che da Barletta conduce a Trani, ed eccoci a piedi del tratturo in mezzo a vigneti conviventi cogli olivi. Questi alberi li veggo floridi, ma non so se in questo basso fondo siano per dare più frasca che frulto. Il fatto deciderà in seguito: per ora sono ancora giovani. Avanziamo il passo, ed eccoci vicino alla contrada di Santo Luca. Notate ora clue noi siamo circondati a dritta e a sinistra da bassi colli, e siamo tuttavia nella dolce vallata. $\mathbf{E}$ bene, questa è appunto che io ho in sospetto, come facente parte di quell' altro avvallamento assai lungo per circa otto miglia cominciando dal sito dell' Ofanto ch'è vicino al ponte crollato. Vi dissi che avrei ritoccato lo stesso argomento, eccomi alla promessa. I miei sospetti sono che forse in remotissimi tempi le acque dell'Ofanto nei momenti delle loro impetuose e strabocchevoli correnti (comunemente piène) divergendo a dritta dell'atluale suo cammino percorrevano questo Iungo e tortuoso avvallamento, che mette foce, per cosi dire, nella vasta pianura delle paludi, dalla quale ne siamo usciti poco fa. La posizione topografica di tutto questo gran canale, e quella dello sbocco che esso fa nelle paludi, mi dà probabilità positive sull' esistenza forse un tempo di questo fatto, siccome anche presentemente si vede quando nel verno le acque dell' Ofanto uscendo dal loro letto, inondano tutti i bassi fondi vignetati della contrada detta fonnèta di Campanile. A meno che non voglia dirsi che questa vallata di Santo Luca, per ciò che riguarda la sua diramazione in croce dal Nord al 
Sud, animata fosse dalle acque che scendono dai luoghi elevali che guardano verso la città di Andria. Dublioso n’è il sospetto per ora. Ma gli è questo uno studio severo a farsi sopra luogo, sotto molti riguardi, per darne un giusto e meritato giudizio. La premura di aver palesato questo pensiero, non è stata tanto per provare l' antico corso delle acque che straripavano, quanto per notare le diversità dei terreni più o meno ricchi in siffatto canale, e le svariate situazioni e posizioni delle sue estremità laterali. Le quali due cose sono importantissime a studiarsi in siffatti luoghi, onde meglio giudicare della vegetazione spontanea nei speciali siti, e della maggiore o minor floridezza nelle diverse coltivazioni delle piante erbacee ed arborce che vi si eseguono. E comechè il territorio barulense non ha montagne, cosi nelle sue pianure, ove frequentano queste ineguaglianze di suolo, le ricerche botaniche debbono essere più attente e prevegenti in rapporto all' importanza, o di qualche specie rara per se stessa, o di altra specie, che mentre abbonda in altri paesi, ivi cioè in quel canale si trovasse per cause tutte naturali e anteriori allo stato delle attuali campagne. Ed è questa la vera ragione filosofica e scientifica per cui vi ho fatto eseguire tanto lungo cammino, che forse a chi non sa vedere, sembrerà strano. In siffatti studii le vedute troppo generali poco si prestano alla ricerca e alla scoverta dei fatti naturali: quindi la necessità dei dettagli. Da che nel 1847 il benemerito sig. Gussone mi espresse il piacere e la sua soddisfazione che io in quell' anno aveva raccolto il 
Thesium humile nelle campagne di Barletta (1), deliberai in me slesso che ben altro metodo conveniva adottare nella ricerca delle piante in queste contrade; e che lungi di limitarmi alla sola raccolta, avrei dovuto notare le particolarità de'sili in rapporto alla natura delle specie e dei paesi ov'esse più comunemente rinvengonsi nello stato di perfetta spontaneità indigena. Di fatti nello stesso anno 1847 e negli altri antecedenti io aveva osservato alle foci dell'Ofanto, cioè alla così delta fiumara, che certe specie di piante fioriscono ivi in agosto ed in settembre, mentre in altri siti esse montano in fiore nel mese di aprile: lo che importa qualtro mesi circa di differenza. Per esempio

(1) Il Thesium humile è pianta di Sicilia e di altri luoghi simili, ed è rara a un tempo, come rilevasi da due opere botaniche del sigg. Gussone, la Flora Sicula e le Plante rariores. Eccone la descrizione dataci dal prelodato Botanico. T. glabrum, caule ramisque erectis adscendentibusque, foliis anguste linearibus enerviis crassiusculis, calycinis laciniis nuce brevioribus demum involutis, floribus, subspicatis quinquefidis bracteatis, nucibus subsessilibus oblongis angulato reticulatis bractea intermedia 2-3-plo brevioribus. Icones: Gussone, planter rariores, tabula 20. In collibus aridis, vel suburgillosis maritimis (in Sicilia). Aprili, Majo. Annua = Caules 2-6pollicares, superne angulati : folia levia, semiteretia, acutinscula, 1-1 1/2 poll. longa, 1/2-2 lin. lata; inferiora integerrima, superiora minutissime serrulata: flores in apice cau'is et ramorum spicati, virescentes, suffulti bracteis 3 ; duabus lateralibus brevissimis recurvis; intermedia serrulata, foliis conforme, 3-4-plo lateralibus longiore, 3-6-lin. longa: calycis lacinia acuta, sub anthesi patentes: muces majuscula. Gussone: Flora sicula: Volumen primum. 
la Ticia pseuda-cracca nasce spontanea nei terreni silicei e alquanto freschi, e spesso l'ho raccolta fiorita in marzo e in aprile ai Bagnoli, al lago di Agnano, a Posilipo, alla Torre Annunziata, a Portici, a Resina, e nel mese di febbraro anche in fiore sull'eremitaggio del Salvatore a Monte Somma, e a primavera alla valle di S. Rocco, ai ponti rossi, alla cappella di Cangiano $\mathrm{e}$ in altri contorni della Capitale. Essa non è certamente pianta palustre; e intanto l'ho raccolta più volte nei siti pantanosi ed argillosi delI'Olanto a fianco della Typha latifolia, dell' Atriplex portulacoides, de' giunchi e di altre piante simili sul cominciare della sua fioritura nel mese di agosto a seltembre. Essa era dunque nata da semi trasportati dalle acque del fiume dai luoghi superiori e più lontani. $\mathbf{E}$ bene, io diceva, quesla pianta non può essere propria di questi siti palustri : laonde essa quivi è pianta peregrina, e in un anno la si trova, in un altro no, come succede ad altre specie, che or ora dirò, secondo le svariate bizzarrie prodotte dalle correnti del fiume. La sua ritardata fioritura proviene al certo da due cause: dall'essere i semi seppelliti per più tempo sotto copiosa arena, o sotto l'argilla, e dal trovarsi in continuata ed eccessiva umidità che sempre suol ritardare la vagetazione di molte piante. La Valerianella echinata, la Specularia speculum, diverse Vicie, e diversi Trifolium, l'Alopecurus agrestis, il Triticum repens, il $P$ 'apaver Rhoas; tutte queste specie le ho rinvenute insieme coi giunchi, colla Typha, coi Carex, colla Salicornia, coll'Atriplex portulacoides e con altre piante palustri. Chi non vede dunque che la presenza di certe date piante in 
talune località non è sufficiente per se stessa a determinare la flora tutta propria di essi luoghi? Siflatto argomento sublime vien trattato colla massima erudizione e filosofia scientifica dall'egregio sig. De Canbolce nella sua Geografia botanica. Ma bastino fin qui siffatte osservazioni. Torniamo in dietro e raggiungiamo la strada nuova che congiunge Trani a Barletta. Eccoci dunque di ritorno alla Città, avendo a dritta e a sinistra vigneti, e frutteti, nei quali campeggia sempre il mandorlo gentile ed il pacifico olivo. Riprenderemo il nostro cammino, ma sotto altro genere di ricerche, cioè quelle della natura de'terreni superficiali e del sottosuolo, siccome in appresso vedremo.

\section{II. ${ }^{0}$}

RAPIDO SGUARDO SULLA NATURA DE' TERRENI.

Se ben vi ricordate, vi dissi altra volta, che lungo il mare, all'Est e all' Ovest della Città i terreni sono sabbiosi. Le sabbie tulte in quei siti sono a base silicea, ove più ove meno grossolana, e ve n' ha della finissima, talvolta anche nera. Eccone l' esempio; noi ora aflondiamo coi nostri piedi, tanta è la sua scioltezza. Ma osservate il suo color chiaro biancastro ? Ciò è dovuto principalmente all'abbondanza sterminata de' frantumi di conchiglie marine, fluviatili e terrestri. Resi questi dall' attrito a volume esilissimo, facilmente somministrano alle piante l' elemento calcàreo, sotto l'influenza svariata degli agenti fisici e chimici. Aggiungete le alghe marine, c tulli gli avanzi orga- 
nici animali e vegetali scaricati dall' Ofanto nel mare e da questo deposti sul lido, e voi vi renderete ragione della copia di terriccio esistente in questi terreni estremamente silicei. Scavate a poca profondità e vi trovate l'acqua. Considerate pure che negli strati superficiali i terreni sono aridi, ed in quelli sottostanti sono umidi costantemente per la interrotta capillarita secondo gli esperimenti di Scnübler sulle proprietà fisiche de'terreni. Riunite tutte queste cause, cioè silice, calce, terriccio, acqua, temperatura piuttosto elevata, e cosi spiegherete in breve il perchè di questa vegetazione cosi rigogliosa che avete sott'occhio: la quale è una pruova soddisfacente sulla possibilità di coltivar quivi la robbia con sicuro e brillante successo. Ciò non dimeno, vi sono località eccezionali, per le quali mai ho potuto indagare le vere cause. Seguitemi, e or ora vedrete. Ecco come si stenta a muovere il passo in queste deserte arene, la di cui superficie è tutta ondeggiata dal vento. Non vi pare questo un esempio in piccolo in confronto di tutto ciò che il signor BeUdant riferisce dottamente nella sua Geologia allorchè parla de la marche des dunes? Come spiegare la cagione di questi tratli deserti di piante? Sono degli spazii più o meno grandi che in questi fertili fondi silicei si trovano di tanto in tanto. E ciò che è originale, si è che in un anno trovate deserto un sito $e$ in un altro anno lo rinvenite fertile. Sarà forse la copia de' principii salini che il mare nei tempi burrascosi vi depone? Principii salini, i quali attenuali sommamente dalle piogge dell' anno seguente, si rendono poi atti alla vegetazione nel terzo anno? E pure in questi 
tratti di silice deserta osservate quà e là piccoli gruppi di rigogliosa vegetazione come tante oûsis. La qual cosa si spiega facilmente colla quantità delle sostanze organiche deposte dal mare, e quindi ridotte in terriccio. Mirate: havvi in quelle piccole oâsis il Triticum repens associato al Pancratium maritimum, alla Scilla maritima, alla Salsola soda, alla Salsola tragus, alla Salsola Kali, al Convolvulus soldanella, alla Cakile maritima, al Cyperus olivaris, allo Scirpus maritimus, all' Holchus lanatus, all' Hordcum murinum, alla Sclerochloa maritima, alla Rotboella filiformis, alla Rotboella cilindrica, al Juncus maritimus, all' Eryngium maritimum, al Tribulus terrestris, al Lotus corniculatus, alla Glycyrrhiza glabra, alla Medicago marina, al Glaucium luteum, all' Euphorbia paralias, alla Euphorbia terracina, all'Euphorbia peplis, all' Helminthia echioides, allo Xanthium strumarium, all'Anthemis maritima, all'Atriplex portulacoides, al Trifolium maritimum e a diverse altre piante. Ora basta de'terreni silicei vicino mare; ed aggiungiamo solamente che alla fiumara e alle paludi abbonda eccessivamente l'argilla. Ivi, ed in particolarità alla fiumara e nelle adjacenze, quando l'argilla è pregna di acqua nel verno, i carri vi affondano sino a tre palmi circa; e quando si dissecca nella state, forma crepacci assai profondi e di una larghezza di più pollici. Passiamo adesso alla osservazione sulla natura de'terreni degli orti, de'vigneti, de'frulteti, e dei seminatorii. Il terreno superficiale di questi fondi in generale è argilloso-calcare-siliceo; e le piante spontanee che più di ogni altra vi si rinvengono (particolarmente nei seminatorii e nei vigneti), sono la Diplotaxis tenuifolia, lo 
Cnicus arvensis, il Cichorium intybus, la Sinapis hispida, l' Hypericum crispum, il Convolvulus arvensis, la Chondrilla juncea, ed altre. $\mathbf{E}$ procedendo innanzi, arriviamo a quella lunga vallata che dall'Ofanto serpeggia sino alla contrada di Santo Luca per varie diramzazioni, e di là credo sino a quella delle paludi. Quivi, cioè in siffatto avvallamento, il terreno è piuttosto argilloso-siliceo-calcareo, e di tratto in tratto dà qualche leggiero segno di marna, siccomé in rarissimi siti appena dà indizio di essere siliceo-argilloso-calcareo e siliceo-calcare-argilloso. Ora usciamo da questa vallata, e saliamo dolcemente le piagge. Eccoci in un' altra zona, ove l'elemento calcareo predomina, ed in generale il terreno è calcare-argilloso, ed inclina spesso al marnoso. Più vi avanzate, più salite su questi bassi colli, e più il calcare predomina, tranne le località eccezionali, ove la composizione del terreno varia da quella predominante. Qui ci fermeremo un poco per una interessante osservazione. La profondità del terreno superficiale ossia del terreno arabile o coltivabile, che dir vogliamo, non è eguale in tutti i luoghi. Cosi partendo dalla Città sino all'avvallamento voi trovate il terreno profondo per due palmi e più. Nella vallata lo rinvenite sino a parecchi palmi; e questa è una delle principali cause che producono la sua speciale fertilità. Dall' avvallamento salendo fin qui ove siamo, otto miglia distanti da Barletta, la profondità del terreno arabile è ben poca cosa, da uno a mezzo palmo, ed in molti siti del tratturo, come sotto i vostri piedi qui redete, si riduce a pochi pollici, ed anche ad un pollice. Ecco perchè la vegetazione è me- 
schina, e gli erbaggi sono poveri e dànno poca rendita. I massari speculatori che vengono a coltivare in questi luoghi colle stesse regole di una masseria a terreno profondo e ricco, spesso alla fine dell'anno s'imbattono nel deficit delle entrate, e se non battono ritirata a tempo, sono guai! Quindi la necessità di riconosecre la vera buona pratica agraria nelle campagne e non già nei libri ; checchè ne dicano in contrario $\mathrm{i}$ critici, che toledando, spulano sentenze (1). In questi terreni calcari per eccellenza e

(1) "In vero , o Signori, quando si veggono sorgere in agricol* tura tali difficoltà, dice il valentissimo chimico MaLagut, e quando " in Francia la maggior parte delle terre stanno nelle mani di po" veri agricoltori i quali non sanno spesso nè leggere, nè scrivere, " che il lavoro urge, che il bisogno incatena, non è forse evilente, "per dirvi tutto ciỏ che penso, che le cose non possono a questo " modo camminar bene? Si, io credo che l' agricoltura della Fran" cia, é quella sovratutto dell' 0vest, non farà serii progressi, se " non quando i proprietarii, ad imitazione degli Inglesi, recheran" no nei campi la loro intelligenza e l' attiviti. Ma fino a clie fuggi" ranno le loro terre e preferirannio il soggiorno delle città in cui « vivono tranquillamente colle rendite loro, sarà misera l’ agricol" tura » (!!!) Avete capito, o Lettore?!.. Fate l' applicazione di questi principii e verità agli altri paesi del globo, e vedete dove vi trovate !! Che vengano gli altri a sostenerci, trovarsi l' agricoltura pratica nei libri ; noi sosterremo e grideremo sempre : Altro è concepir $l$ 'aratro a tavolino, altro è manegyiarlo nelle diverse qualitè di terreni secondo le regole della meccanica agraria!! Diversa è la coltivazione di una pianta in un giarlino da quella in una masseria ove devesi bilanciar tutto in armonia coll' introito. Altro è descrivere un vegetabile, altro è coltivarlo e convertirlo in monela. I proprietarî e la società intera vogliono il grano nel granaio, ed hanno ragione. Lo vogliamo capire, sì 0 no ? E per giungere a questo 
molto secchi abbondano di più le piante labiate tanto utili alle api, tra le quali si distinguono sommamente il Thymus capilatus, il Thymus Zygis, il Thymus Acynos ed altre piante della stessa famiglia. Noi fin qui abbiamo dato un rapido sguardo alla natura superficiale del terreno. Faremo altreftanto pel soltosuolo: e senza camminare di più per le stesse campagne che percorremmo altra volta, ricorderemo solamente a noi stessi le principali contrade delle medesime, accenneremo le particolarità del sottosuolo, e porremo termine alle nostre osservazioni su i terreni. Dall'avvallamento o canale, che dir volete, fino dove stiamo noi; e da questo punto più in sopra e per tutti i lati, a dritla e a sinistra si rinvengono estesissimi ed assai profondi banchi o depositi di tufo calcareo, di tal che vi si possono cavare cantine e grotte, ed anche le stanze sotterrance per fabbricare e cuocere il pane siccome un anno vidi nel feudo del Sig. D. Ferdinando Cafiero; e presso Canosa nel suddetto tufo si cavano grotte ampie a più gallerie per conservare il viuo, e sopra si coltivano cereali, biade, legumi, fichi, mandorli, ed olivi. E voi sapete come vicino Napoli, a Posilipo, a Capodimonte, a Cardito di Caivano, sotterra sono le grotte, e sopra le coltivazioni erbacee ed arboree. Da Barletta dunque andando

scopo, è mestieri la dimora e lo studio nei campi, onde possiamo impadronirci delle loro qualità e metterle a profitto nostro e degli altri, mercè l'ajuto e i lumi delle Scienze Naturali. Lettore mie carissimo, o vi piace o non vi piace, io vi parlo chiaro. La presun zione nei gabinetti da studio è una brutta cosa: la modestia e la sincerità nell' nomo di scienze è un gran tesoro. 
verso Canosa, spesso trovate gran deposito di tufo, il quale o giunge sino alla superficialità del suolo, o è coperto da uno strato di terreno argilloso calcare, o da uno strato di crosta, della quale parleremo da qui a poco. V'ha il tufo bianco, il giallo, il paglino, ed il rossigno. Vi si trova il tufo puro e a grana legittima, e quello frammisto più o meno a conghiglie fossili, ch'è un poco men pregiato dell'altro. Ed a proposito di fossili, in generale, debbo dirvi che nel tufo di Canosa soventi volte se ne trovano de' grossi e dei belli. Nel 1844 ivi ne comprai uno, che al ritorno in Napoli l'offersi al Signore Professore Scaccin, che acceltò volentieri. Se amate notizie dettagliate su questa branca scientifica, consultate le opere del Signor Abate Giuseppe Maria Grovene, da Molfetta, e di tutti gli altri nostri Professori viventi, i quali dottamente ne trattarono nei loro pregiati lavori di Geologia e Paleontologia; Scacchi, Costa ed altri: e de' trapassati Cagnazzi, Picld, Tondi ec. ec. Se poi, muovendo da Barletta, battete la via di Andria, invece di tufo rinvenite carbonato calcare a grana fina, bella e lucida, ed a superficie piuttosto liscia. Vien desso chiamato pictra viva e serve alla fabbrica delle case e di altri edifizii, allo stesso modo che si adopera il tufo. La calce, che se ne oltiene, ̀̀ ottima. Ora è utile (per la più facile riconoscenza delle varie contrade delle campagne barulensi) il dirvi che il petraro, cosi detto, trovasi lungo la via di Andria per varie diramazioni cominciando dal casino di Binetti e procedendo verso questa Citta; e le cosi dette tufare per diverse linee rinvengonsi sulla via di Canosa, dalle vicinanze di Montalino 
in sopra. Tutte due queste estese contrade sono aride, e l'acqua più o meno salmastra s'incontra a moltissima profondità. Talvolta vi si trova qualche vena di acqua dolce, che forma la fortuna e la ricchezza di quelle masserie che la posseggono. E questo falto mi sembra provenire facilmente dal perchè le acque sotterranee di quei luoghi (che sono i più fortunati) scorrono per strati terrestri meno carichi di sostanze saline. Avendo finora parlato della natura de' terreni arabili e loro diversa profondità, non che del sottosuolo nelle contrade le più elevate; è mestieri tenervi parola di quell'altro sottosuolo che si rinviene in quasi tutti i terreni messi piuttosto in piano. Chiamasi comunemente crosta, come poc'anzi vi dissi, ed abbonda da per ogni dove. Essa in sostanza non è che un carbonato calcare alquanto impuro, perchè dà segni de’ caratteri marnosi, ed il suo colore è biancastro sporco. Ve n'ha degli strati piuttosto friabili, che sono facili a cavarsi anche colla zappa; e ve n' ha di quelli così duri, ch'è necessario adoperare un forte piccone maneggiato da polso fermo. Di tutti gli strati di crosta quelli i più duri si trovano quasi in tutti i luoghi. La crosta rinviensi dopo lo strato di terra arabile alla profondità di due palmi, poco più o meno, secondo la conformazione geologica degli strati calcari, e degli scarsi o copiosi depositi di terreno argillososiliceo che le correnti parziali delle acque nei diversi luoghi col tempo vi deposero. Si è nella crosta che si cavano le formelle o fosse, che dir si vogliano, nelle quali si piantano i novelli vigneti. In essa si abbarbicano potentemente le radici degli alberi fruttiferi e delle viti, le quali 
producono quei vini neri, robusti, e generosi, e che sono conosciuti realmente da tutto il mondo, sono ormai parecchi anni, per cagione della infausta malattia delle uve. Io non ho per ora esperimenti ed analisi chimiche per sapere, se i silicati di potassa (di cui si diletta la vite dalla sua adolescenza in poi, secondo gli studii pratici de' moderni Agronomi) esistano copiosamente in natura nella crosta; o se, dopo cavate le fosse per le vigne, e quindi piantati i tralci, succedendo necessariamente la mescolanza del sottosuolo col terreno coltivabile, i sudetti silicati solto l'azione della calce, ch'è nella crosta, siano messi in libertà col tempo dall' argilla, nella quale erano rinchiusi, e quindi sciolti dalle acque, giungano sino alle radici delle viti, traversando un terreno reso sufficientemente soffice e sciolto, mediante l'antecedente cavamento, la mescolanza delle terre, e le ulteriori coltivazioni? "Suivant Liebig, la " chaux agirait surtout en accélérant la déségrégation des " silicates alumineux et alcalins, et en mettant ainsi à la " portée des racines les principes alcalins, indispensables “ au commencement d'une nouvelle végétation. Il est cer" tain, d'après Fucus, que, lorsque la chaux vive reste, « sous l'nufluence de l'humidité, en contact avec de l'ar" gile, elle s'y combine, et qu'un des effets principaux de " cetle combinaison est la mise en liberté des alcalis que " cette argile renferme à l'état de silicates. » Grandroin ottimo Agronomo e gran Chimico riferisce le anzidette parole. Sicchè la mia proposta, la mia dimanda, il mio dubbio, non sembra senza fondamento. Una pratica piuttosto lodevole è messa in uso nei novelli vigneti di Barletta: ed 
eccola. Nel verno si pongono i tralci: in primavera nel terreno sciolto si affidano poponi e fagioli, senz’altro soccorso che quello della scarsa piogrgia estiva. I corrispondenti ricolti sono copiosi ed eccellenti. D'onde ciò proviene? Mille ragioni fisiche e chimiche militano a favore di questo bel fatto. Non vi parrà intanto discaro, o Lettore, ii farvi notare che nel cavamento della crosta, spesso accadono disgrazie in persona dei contadini che hanno la sventura di buttar sangue dalla bocca. Ed in vero un terreno cosi duro, cavato da quattro a sei palmi da un campagnuolo a gambe aperte sulla fossa, e tutto grondante di sudore nel mese di agosto, dev' essere causa sufficiente da far perdere la vita a chi non abbia petto forte e polso sostenuto solto un' ardente canicola col capo chino per più ore di seguito. Fatevi a contemplare la posizione di quei poveri vignaiuoli, e vedrete quanto lor costi il pane quotidiano per le loro rispettive famiglie!! Passiamo avanti a tanto dolore !. . Pria di por fine alle osservazioni sulle diverse qualità di terreni, mi lusinga il narrarvi, a tal proposito, ciò che mi fu dato vedere, son ricordo bene se nel 1843 o nel 1844. Nella villa Bonelli presso Barletta si cavava un pozzo bastantemente profondo. Ivi mi avvicinai per osservarne il terreno cavato. Il quale ammonticchiato, com'è solito in simili casi, ne offriva qualità diverse: cioè eravi il terreno arabile mescolato alla crosta friabile secca: la crosta dura: una terra calcare umida e ammollita come la pasta: una specie di marna calcare carica di rognoni calcari e di ossido metallico, percio era rossastra e giallognola: una ghiaja grossolana ed 


\section{$-42-$}

un'altra fina, e la prima di queste due era sommamente carica di conchiglie, di elici, e di frantumi di creta: finalmente una marna assai argillosa cosi compatta e a grana tanto fina e gentile, che sembrava una vera argilla pura; essa aveva un colore verdastro chiaro e strisciato di tanto in tanto di vene rosse, che alludevano senza dubbio alla presenza di qualche ossido di ferro. La saggiai cogli acidi che vi produssero una effervescenza alquanto pronunziata. Tulte queste terre erano confusamente ammonticchiate, e ne raccolsi taluni saggi. Avrei voluto scendere abbasso al pozzo per osservare con più esattezza e da vicino i diversi strati; onde notare la loro giacitura, vedere la disposizione graduata de' diversi terreni, cioè chi più sopra e chi più sotto, e finalmente segnare la spessezza di ciascuno strato. La qual cosa avreble formato un complesso di notizie positive sulla natura e situazione di quei materiali nelle parti più ime di quel suolo, in rapporto all'influenza che essi terreni eserciterebbero sulle radici delle varie piante arboree. Ma non potei ciò eseguire per mancanza di mezzi opportuni onde scendere nel pozzo senza pericolo alcuno. Questi cenni sulla natura di quei terreni inferiori io ho voluto esporvi, alla meglio, perchè spero col tempo potessero i medesimi richiamare l'attenzione di coloro che occupandosi di Geologia, siano per dare lumi soddisfacenti non solo dal puro lato scientifico, ma particolarmente agrario per la collivazione in grande delle piante arboree in quelle contrade. Abbiamo dunque esauriti due de' tre punti che da principio promettemmo dover brevemente trattare; cioè uno sguardo sulla posizione topografica delle 
campagne di Barletta, e l'altro sulla natura de' terreni. Voi mi avete onorato della vostra compagnia in due passeggiate; la prima più lunga della seconda; ed io grato alla vostra cortesia, o Lettore, mi feci un pregio di servirvi come meglio mi riusci. Quelle passeggiate erano indispensabili, perchè si trattava di fatti che solamente sopra luogo si possono vedere e studiare. Ora resta il terzo punto, vale a dire le conoscenze del clima, le quali non potendosi fare in un giorno, nè in un solo anno, è giuosc forza che ci ritiriamo in Ciltà, ed ivi, chiusi in un m̧binetlo da studio, mediteremo sulle opere di colui cho per ben venti anni della sua vita si consacrò allo studio del clima pugliese. Laonde onoratemi di vostra compagnia, e domani sarò pago della compiacenza che avrete alla lettura di libri istruttivi e pieni di dottrina.

\section{III. $^{\circ}$}

Notizie sul Clima pugliese, E PRECisamente

SU QUELLO DELLA PEUCEZIA

Eccoci, o Letlore, finalmente a riposare, dopo due lunghissime passeggiate, delle quali la prima costa di circa cinquanta miglia, e la seconda di miglia otto in dieci a un di presso. Non potevamo farne a meno, per avere uno schizzo delle campagne di Barletta. Ora studieremo un tantino il clima, non già con osservazioni dirette (poichè a ciò fare, ci vorrebbero non meno di dieci anni), ma frugando nei lavori di un uomo dotto che fa immenso onore alla Provincia di Bari e alla sua patria Molfetta. Inten- 


\section{$-44-$}

do parlare dell' immortale Arciprete e Cavaliere Signor Giuseppe Mlaria glovene, la di cui perdita fu un danno positivo per la Provincia e particolarmente per la gioventù che faceva tesoro de' suoi dettati e dei suoi lumi. Socio egli si era dell' Accademia delle Scienze della citta di Modena; e se onorava la sua patria, non minor lustro accresceva all'Italia. Fiori nel secolo decimo oltavo e nel cominciamento del corrente, e scrisse varii pregiatissimi lavori agrarii, particolarmente sugli olivi, taluni botanici, ed altri molti in metcorologia, che sono appunto quelli i quali formeranno l'oggetto delle nostre investigazioni e studii in questo pacifico gabinetto. Ma voi direte; che ha che fare Molfetta con Barletta? Adesso vi servo. In Barletta, per quanto è a mia notizia, nessuno si è occupato di meteorologia; studio severo che esige la più grande pazicuza e costanza pel periodo di lunghi anni. L' Arciprete grovene si occupò del clima pugliese ed in particolarità di quello della Peucezia. Barletta dista da Molfetta quindici in sedici miglia, che fanno una breve distanza. Queste due Cilla sono poste presso a poco sullo stesso grado di latitudine settentrionale $410^{\circ}$ colla differenza di varii minuti primi fra loro: e verso il $140^{\circ}$ di longitudine tale a partire dal meridiano di Parigi. Tra l'una e l'altra Città non vi sono nè fiumi, nè laghi, nè boschi, nè montagne; meno che una ineguaglianza di suolo intersecato e variato da fertili vallate e da collinette amene, sulle quali non vedete altro che vigneti, mandorleti, e oliveti in quanlitì e quasi tulti in uno stato vegetativo di una grande floridezza, e alternati di tanto in tanto da campi addetti alla 
seminagione. Queste condizioni locali, prese in generale, da Barletla a Molfetta, sotto il rapporto della vegetazione in massa delle suindicate piante arboree e di altri vegetabili, esse, io dico, sono sufficienti per se slesse ad assicurarvi di un clima quasi del tulto identico, trame qualche frazione eccezionale rappresentata dai diversi angoli ottusi e dalle larghe curve che tutta la costiera marina presenta all' occhio dell' osservatore, il quale in un legno sopra mare dar volesse uno sģuardo rapido sulla posizione topografica del littorale della Peucezia. Noi dunque possiamo essere sicuri nell' applicare al clima di Barletta tutti i calcoli, osservazioni, e studii meteorologici fatli per ben venti anni in Molfetta e suoi contorni dal benemerito Ginseppe Glovene, poichè la vegetazione è quasi tutta dello stesso caratlere nelle campagne delle due Cilli, tranne forse poche specie particolari di piante indigene che rinvenir si potessero nei pascoli sassosi che sono tra Molfetta e Corato; i quali, avuto riguardo alla loro posizione topografica, dàno indizio di qualche specialila, come io medesimo osservai sopra luogo nel Maggio 1851, grazie alle cortesie ed amabilità prodigatemi cordialmente da’ Signori Dc Judicibus di Molfetta, i quali mi fecero visitare quelle amene e ridenti campagne. Che se aggiungiamo a queste considerazioni generali il fatto della natura calcarea predominante, tanto nell' agro barulense che in quello molfettese, noi arremo un altro appoggio di più a riconoscere l'identilà di carattere nella vergetazione in massa de' due paesi. Sicchè senza dilungarci margoiormente, proponiamoci prima in breve quel poco che ne importa di essen- 
ziale sapere, cioè i principali agenti del clima sulla vegetazione tutta delle campagne barulensi. Noi studieremo approssimativamente la quantità di pioggia annuale, i venti dominanti, la gradazione di temperatura, e qualche altra influenza climaterica speciale che le vigilie di quel dotto uomo raccolscro e registrarono nei suoi lavori; non escluse le nostre piccole osservazioni pratiche sopra talune cose meteorologiche. Egli nel suo prospetto comparato della pioggia della Puglia esprime taluni pensieri che bisogna leggere attentamente e col cuore. Leggiamo, mio caro Lettore, leggiamo. "Il quadro, che io presento al pubblico, della pioggia, che dal cielo cade su di una Citù posta nel litorale della grande piamura della Puglia, qual' è Molfetta, risultato della osservazione di venti anni ". Aito qui per poco. Avete letto osservazione di venti anni? $\mathbf{E}$ noi intanto pretendiamo che si scriva e si pubblichi per le stampe, ed in pochissimo tempo, una istituzione agraria applicabile a tutte le campagne del Regno di Napoli, come se fosse un paese tutto monotono e senza varietà! Abbiamo dimenticato quale influenza esercitano sulle nostre campagne gli Appenini, partendo dal fiume Tronto sino a due Capi, di Leuca l'uno e di Spartivento l'altro ? Abbiamo messo in dimenticanza quanto ne disse dottamente il Sig. Cavalier TENoRE nella sua Geografia botanica del nostro Regno? $\mathrm{Ci}$ è uscito di memoria quanto ne scrissero molti valenti conciltadini relativamente alle diverse campagne di ciascuna Provincia ? Leggete un poco le Memorie agronomiche pubblicate nel Giornale enciclopedico del Regno e quelle altresi inserite negli Alti dol Reale Istituto d' In- 
coraggiamento, e vedrete quanto ci è da studiare nelle Provincie, e come disagevole cosa sia il compilare e tessere una buona istituzione agraria pel Regno, poggiata sopra i falli e non castelli in arja! Ignoriamo forse gli svariati prodolti agricoli del nostro ricco paese e le loro corrispondenti industrie ? Non è a nostra conoscenza la diversità specchiata che passa tra l'influenza del clima sulle coste del Tirreno e quella del clima sulle coste dell' Adriatico? Abbiamo dimenticato le gradazioni di questi due climi a misura che ci avviciniamo agli Appennini, e viceversa, da essi insino al mare? Questi sono studii che si debbono fare nelle campagne e nelle loro rispettive località, e non già né caffè, nelle farmacie, e nei magazzini serali ove si fabbricano panni e castori di ogni qualità!! L’ Agricoltura vera sta nei canıpi io sostengo fortemente contro l'opinione dei palloni volanti. Là solamente si studia e si perfeziona; e dopo la esatta conoscenza de' fatli naturali e delle svariate pratiche per ciascuna Provincia, allora si potreble dar cominciamento all'opera. Non dimenticate che la convenienza di ogni coltura, dice il grande e dotto chimico MALAGUTI, è sempre subordinata a circostanze che sfuggono alle previsioni teoriche e che risultano sia dalle località, (!!!) sia dalle esigenze industriali e commerciali. Non è del nostro carattere il copiare e rubare le stesse canzoni teoriche nei libri altrui per scrivere una istituzione agronomica!! Canzoni che sono state dette e ridette le mille e mille volte sino alla noja! Le smodate ed irragionevoli esigenze de'toledatori si abbiano alla pur fine un freno: e se veramente vogliamo il bene 
del nostro paese, cessiamo una volta di sciupar tempo alla lettura di quelle tali operacce romantiche oltramontane che scompigliano la mente e guastano il cuore della mal consigliata gioventù! Diamoci agli studii severi e utili. Agricoltura; Meteorologia; Meccanica; Geografia botanica ed agraria; Commercio; Industria; Economia; Tecnologia; Pastorizia; Veterinaria. Questi sono i veri studii utili che formano gli uomini ed arricchiscono la società! Non sarebbe per noi piultosto utile che un giovine si occupasse o di setificio, o di enologia, o di caseificio, o dell' industria della cera e del mele, o di quella della manna, o della navigazione, o della coltura dello zafferano, o di quella dell' olivo, anzichè consumare gli anni nella pubblicazione de'sonetli e delle ridicolaggini de' paesi stranieri? Per far dunque una istituzione agraria applicabile alle nostre campagne, bisogna prima conoscerle a fondo nei diversi siti; conviene innanzi tutto fare goli studii comparati sopra ciascuna pianta e rispetliva coltivazione tra le diverse Provincie, e quindi riunire tutte le notizie ed i fatti, ed esporli poi in un sol corpo di opera scientifica bene ordisata ed esposta in modo tale, da essere adottata alla intelligenza di tutti i proprietarii non solo, ma che in essa opera ciascun possidente trovi quel complesso di regole agrarie tutte proprie pel suo paese, quantunque le piante siano le stesse. Come vorreste voi, per esempio, applicare le medesime regole di Terra di Lavoro, in Puglia ed in Calabria per la coltivazione delle viti, e viceversa, senza fallire nella vostra impresa? Ad ottenere dunque siffatta opera occorrono due condizioni : $10^{a}$ viaggiare e studiare per le cam- 
pagne: $2 .^{a}$ il tempo materiale necessario a tutte le cose. I possidenti rispeltano ed amano l' indispensabile trascendentalismo nelle opere di Scienze Naturali; ma in fatti di Agricoltura preferiscono un tantino di vedere l'aumento quantitativo del grano nel granajo, dell'olio nella cella olearia, delle balle di lana nei magazzini ecc. alle troppo elevate osservazioni filosofiche e progetti aereostatici di coloro, i quali si lusingano sventuratamente che la dottrina somma di un qualsiasi uomo possa creare ed alimentare in Jui l'universalità di tutte le dottrine!! Ed hanno ragione i possidenti; poichè senza pane non si vive!!! Ma basti fin qui ciò che ho delto: io mi sono dilungato per necessità, e me ne trovo contento e soddisfatto nella mia coscienza. Verrà col tempo, verrà senza meno il disinganno di coloro che pensano in contrario!... Riprendiamo ora il filo delle parole del nostro benemerito Arciprete grove$\mathrm{NE}$, e poi studieremo quel tanto cli' egli ottenne in venti anni di osservazioni sulla quantità delle piogge nella $\mathrm{Pu}$ glia. Egli dunque, l'Autore, continua a dire così:-e della piogyia su di un' altra Città ancor della Puglia, ma messa in collina, qual' è Altamura, risultato di quattro anni d'osservazione; e finalmente di quella che cade su di una terza Citlà, che, se non appartiene propriamente alla $P_{u-}$ glia, essendo per verità negli Irpini, ne è però quasi al confine e per ventura trovasi situata su di un monte nel centro degli Appennini, risultato delle osservazioni di undici anni: un tal quadro, dico, se è di una utilità per la Regione $\mathbf{P} u-$ glicse, può ancora non essere dispiacevole a coloro, $i$ quali amano conoscere una parte del fisico di un paese poco cono- 
sciuto, e che per altro fu celcbre nell Ancichicì, e lo è anche a'di nostri (vedremo se a corto o a ragione, e per qual ragione) per la sua aridità e secchezza. Ed acciocchì a tali osservazioni si possa allaccare quel grado di confidenza che è necessario, senza dir parola della mia esattezza, e molto meno della esattezsa degli egregii miei amici signor Arcidiacono Cagnazzi di Altamura, e doltor Don Giovanni Zerella di Ariano, dirò soltanto, le osservazioni essere state praticate col metodo dell'illustre fu Abate ToALDo, e le mie con vasi e misure gentilmente da lui mandatemi, le quali gelosamente conservo qual yegno dell' amicizia di un cosi celebre e benemerito nostro Socio. Ed a fare cosa grata ai LetIori vi ho unito ancora la pioggia di Teramo Cilli dell' allo Abbruzzo, frutto delle osservazioni esattissime, per due. anni, dell' oltimo mio Amico signor D. Orazio Delfico. Ora che abbiamo visto, assai bene, quanto zelo poneva il Grovene negli utili studii metcorologici, è giusto l'osservare il quadro riassunto delle sue osservazioni di venti anni sulla quantità della pioggia annuale nella $\mathbf{P u -}$ glia Peucezia. E perchè siffatto sunto si renda giovevole a noi due e a tutti coloro che non vorranno guardare con occhio bieco il nostro studio agrario, io stimo indispensabile la lettura di questo passo dell' Autore il quale osservava e studiava i fenomeni naturali colla massima esattezza. Sentiamo che dice: Io ho voluto variamente tormentare, a dir cosi, quella mia tavola della pioggia di Miol fetta, ed ho voluto calcolare gli anni, non incominciando già dal gennajo, ma bensi dal marzo al febbraro, e poi incominciando dal giugno al magyio, e poi finalmente dal settembre all'agosto, 


\section{$-51-$}

unendori le osservasioni del corrente anno 1804 a completare $i$ venti anni. Ed ho trovato, che calcolandosi l'anno della pioggia da settembre ad agosto si hanno le massime differenze tra anno, ed anno, e che all' incontro le minime differenze si trovano allora che l'anno si calcola all'ordinario da gennaro a decembre. In fatti calcolandosi nella prima maniera si trova il massimo della pioggia essere di 30:8:8, qual fu l'anno calcolato da settembre 1791 al 1792, ed il minimo essere di 10:11:5, quale appunto fu l'anno da settembre 1801 ad agosto 1802; e la differenza tra questi termini è di 19:9:3, differenza di presso a due terzi, e pare sicuramente cnorme. All' incontro poi il massimo, nello stile ordinario da gennaro a decembre, si trova essere di 29:7:4, il minimo di 13:9:8, la differenza tra i quali è di 15:9;8.= La conseguenza intanto, che da ciò io voglio inferire è questa. L'anno pluviale agronomico doversi incominciare da settembre ad agosto, almeno per la Regione Pugliese. Disse bene Teofrasto, e dopo lui lo hanno ripetuto tutti gli agronomi, che ANNUS FRUCTIFICAT NON TERRA. Sono le meleore occorrenti in un anno, le quali più o meno favorevoli alla vegetazione producono la fecondità, o la sterilità, e tra le meteore quella che piu influisce alla vegetazione, è la pioggia. Ma calcolandosi a questo modo la pioggia da settembre cioè all' agosto, si conosce in tulla la maggiore estensione, in che un anno differisca dall' alıro, e così è facile render ragione tal volta della sterilità o fecondità di un anno. E pare in fatti, che la terra nel 1802 non avesse voluto dar frutti; che se si vegga nella tavola della pioggia in quell'anno, si trova esservi caduti pollici 18:0: $\frac{1}{12}$ di linea di pioggia, non 
si pensa, che di tanta sterilità fosse stata cagione la scarsezza della pioggia, e si anderanno arzigogolando altre cause, Dio sa se vere, poichè finalmente 18:0: $\div$ poco si discostano dal medio. Ma quando si calcola da settembre ad agosto si trova esservi caduti, cosi come ho detto, pollici 10:10:5 ed allora è ben facile riconoscere la causa del male. E gli antichi nostri Pugliesi dal settembre appunto incominciavano $l$ ' anno, e poichè erano addetti all' agricoltura avevano ragione. Fino all' agosto incominciano gli alberi ad entrare in nuovo succhio, ed a prepararsi, per cosi dire, ai frutti del nuovo anno: verso li principii di settembre incomincia ad osservarsi un certo disturbo, e movimento nel barometro, $e$ di settembre incominciano le semine ed i lavori, e tutt altro che serve per la fruttificazione del nuovo anno; nè mi anderebbe a sangue, che l'anno incominciasse dai 23 settembre, ma se fosse a desiderare, desidererei, che l'anno pluviale agronomico incominciasse piuttosto dai 23 agosto. - E pur vero per altro che nell'anno da gennajo a decembre si ha una certa compensazione di pioggia onde ne risulta la minima differenza, cosa questa, la quale può avere li suoi utilissimi usi, poichè essendo vero che nell' anno da gennaro al decembre si compensano le piogge, se si troverà che nell' anno da settembre ad agosto sia caduta molta o poca pioggia, si potrà pronosticare, che debba il più, o il meno compensarsi nei mesi susseguenti di settembre, ottobre, novembre, e decembre. Cosi ancora il periodo di otto anni si trova essere sensibilissimo calcolandosi la pioggia da gennajo a decembre. In fatti sommando la pioggia caduta dal 1784 al 1791, si trovano pollici $148: 7: 0$, e sommando dal 1792 al 1799 si hanno 
pollici 1 46:1:0, somme presso a poco uguali. $\boldsymbol{E}$ parimenti sommandosi dal 1788 al 1795 si hanno pollici 158:7:4, e dal 1796 al 1803 si trovano pollici 150:1:9, somme neppure gran fatto differenti. Ma in vece del periodo di otto anni calcolandosi da seltembre ad agosto si trova chiaramente, che in ogni quadriennio sogliono in pieno le piogge alzarsi alternativamente, ed abbassarsi a riserva di qualche anomalia. Ecco una picciola tavola delle piogge cadute da settembre ad agosto, divise per cinque quadriennii.

Da Settembre ad Agosto Pollici Linee Dodicesimi

\begin{tabular}{|c|c|c|c|c|c|}
\hline deI & & del & & & \\
\hline 1784 & - & $178 b^{\circ}$ & 14 & 11 & 与้ \\
\hline 1785 & - & 1786 & 19 & 10 & 0 \\
\hline 1786 & - & 1787 & 16 & 2 & 5 \\
\hline 1787 & - & 1788 & 14 & 8 & 9 \\
\hline
\end{tabular}

Da Settembre ad Agrosto Pollici Linee Dodicesimi

\begin{tabular}{rrrrr} 
del & \multicolumn{5}{c}{ del } & & & \\
1788 & $-\quad 1789$ & 24 & 10 & 11 \\
1789 & $-\quad 1790$ & 23 & 4 & 0 \\
1790 & $-\quad 1791$ & 27 & 3 & 2 \\
1791 & $-\quad 1792$ & 30 & 8 & 8
\end{tabular}




$\begin{array}{cccccc}\begin{array}{c}\text { DaSeltembre } \\ \text { del }\end{array} & \text { ad } & \text { Agosto } & \text { Pollici } & \text { Linee } & \text { Dodiesimi } \\ 1792 & & \text { del } & & & \\ 1793 & - & 1793 & 17 & 7 & 6 \\ 1794 & - & 1794 & 18 & 2 & 6 \\ 1795 & - & 1795 & 14 & 14 & 8 \\ & & 1796 & 14 & 4 & 8\end{array}$

\begin{tabular}{|c|c|c|c|c|c|}
\hline Da Settembre & ad & Agosto & Pollici & Linee & Dodicesimi \\
\hline del & & del & & & \\
\hline 1796 & - & 1797 & 20 & 10 & 1 \\
\hline 1797 & - & 1798 & 14 & 8 & 9 \\
\hline 1798 & - & 1799 & 21 & 4 & 11 \\
\hline 1799 & - & 1800 & 24 & 9 & 0 \\
\hline $\begin{array}{c}\text { Da Settembre } \\
\text { del }\end{array}$ & ad & $\begin{array}{c}\text { Agosto } \\
\text { del }\end{array}$ & Pollici & Linee & Dodicesimi \\
\hline 1800 & - & 1801 & 17 & 0 & 6 \\
\hline 1801 & - & 1802 & 10 & 10 & 5 \\
\hline 1802 & - & 1803 & 23 & 7 & 1 \\
\hline 1803 & - & 1804 & 16 & 8 & 1 \\
\hline
\end{tabular}




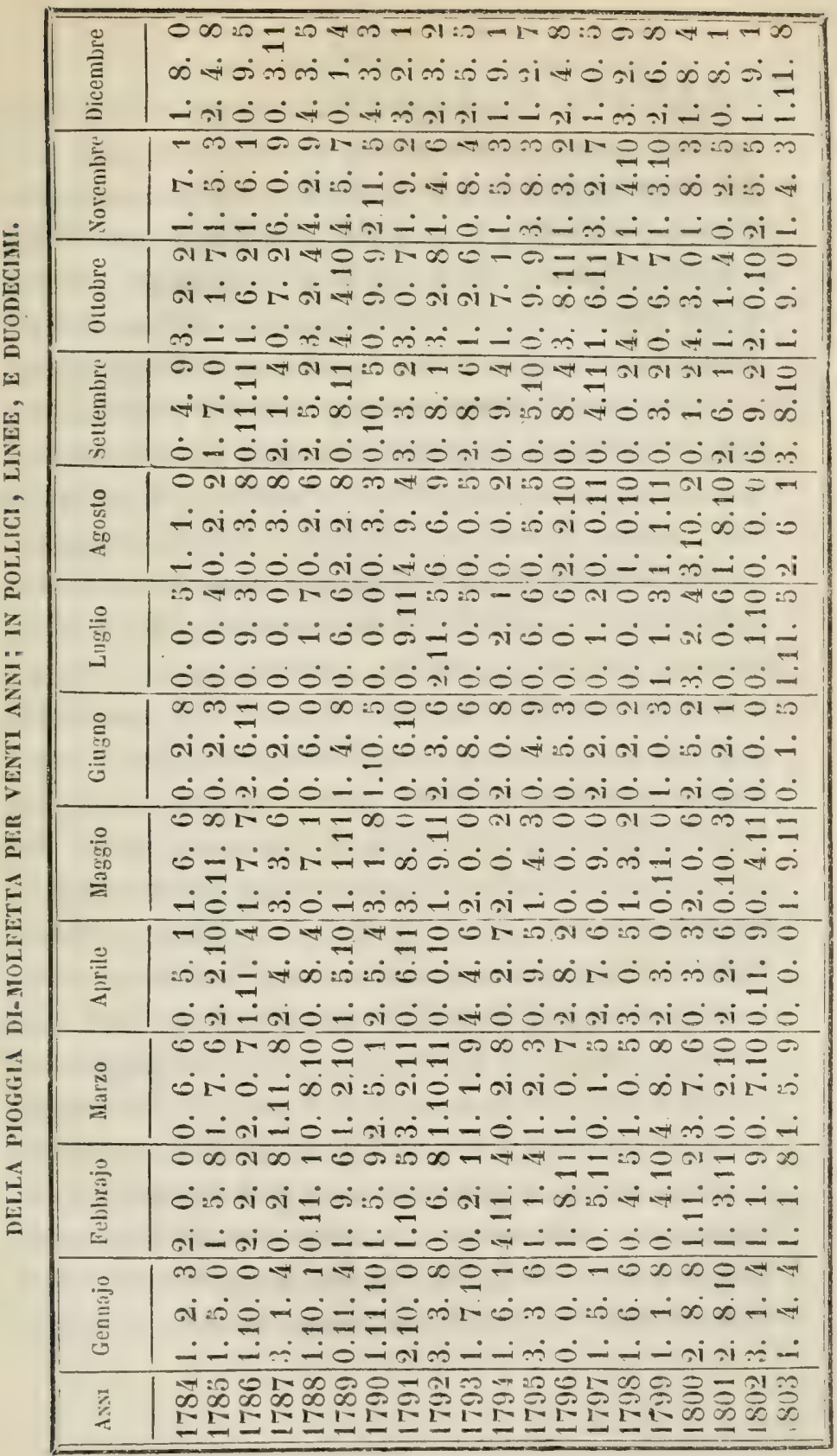


Per altro ì antica osservazione (continua a dire il sig. Aba1e), che in ogni quadriennio si dà un nuovo tuono dirò cosi alle meteore, ossia, che si faccia un rialsamento, o abbassamento; e Plinio avrà voluto ciò indicare, come indicò certamente il periodo di otlo anni, allora che disse. "In di" candum et illud, tempestates ipsas ardores suos habere " quadrinis annis, et easdem non magna differentia reverti " ratione solis, octonis vero augeri easdem centesima re" volvente se luna ". Il quale passaggio per altro non so se si legga oggi cosi come Plinio lo scrisse, ed è certamente molto oscuro. Sarebbe or a dire del ventipiovolo Pugliese, che è l'Est appunto, vento che gli viene dalla Grecia ec. ec. Basta ciò per ora. Noi possiamo ritenere siffatti studii meteorologici del sig. Grovese come un tesoro di cognizioni climateriche per la Puglia Peucezia. Due grandi conseguenze emanano direttamente dalle dotte, esatte, e minute osservazioni del nostro Autore. La prima si è, che la necessità di mettere a calcolo la compensazione del minimum e del maximum di pioggia tra i differenti mesi e tra gli stessi anui, fa pienamente eco a quanto l'illustre Conte De Gasparin dice nella sua vasta e dottissima meteorologia campestre, volume secondo. Egli, il DE GASPAlix espone che la ripartizione de' giorni di pioggia secondo le stagioni è cosa che deve formare il nostro principale studio. C'est principalement la répartition des jours de pluie selon les saisons quiil nous faut considérer. Santa osservazione, nella quale si era bene avanzato l'Arciprete Grovene. Ed in effetti, supposti due paesi, aventi presso a poco la medesima quantita di pioggia annuale; ed in uno 
di essi il maximum di pioggia cadesse in pochissimi mesi ed il minimum in molti mesi dell'anno; ed in un altro di essi paesi la totale quantità di pioggia fosse più egualmente distribuita nel corso dell' annata e particolarmente in quello dei mesi, e delle stagioni in rapporto alle diverse specie di piante coltivate; ne segue, che a parità di circostanze si troverà meglio la vegetazione in massa del secondo paese, anzichè quella del primo. Il sig. DE GasPaun all'articolo pioggia si distende assai in svariate, dotte, e brillanti osservazioni di questo genere, e ripartisce siffalta materia sotlo diversi titoli importanti: cioè $10^{\circ} D i-$ stribution des pluies : 2. ${ }^{\circ}$ Quantité totale de pluie annuelle: 3. ${ }^{\circ}$ Réparlition des pluies selon les saisons: 4. ${ }^{\circ}$ Répartition des pluies par mois; ed è quivi che l'Autore Francese cita gli esperimenti fatti a Molfetla, ad Altamura, e ad Ariano, ma non fa menzione nè del sig. Giovene per la prima Ciltà, nè del sig. Cagrazzi per la seconda, nè del sig. Zerella per la terza. Invece fa menzione delle Memorie della Società Italiana di Scienze, residente in Modena, le quali Memorie furono scritte dal sig. Giovese: e se il De Gaspariy fa cenno degli altri meteorologisti, tra i quali Scrow, Cotte, Poitevin, n' Honbres, Tardy, Kuntz, ed altri valenti uomini che si occuparono della meteorologia nei diversi paesi di Europa, ed in ispecialità di Scrow, che scrisse sul clima d'Italia; era giusto nominare nella sua pregiata opera (ch'è la vera Filosofia della Scienza agraria) anche l'Arciprete Giovene che onorerà eternamente la Puglia, il Regno di Napoli, l'Italia intera : 5. Nombre de jours de pluie: 6. Répartition des jours de pluies selon 
les saisons (Lellore, riflellete bene che al numero $3 .^{\circ}$ l'Autore parla della ripartizione delle piogge secondo le stagioni; e adesso parla della ripartizione dégiomi di pioggia secondo le stagioni: lo che importa due cose diverse): 7. Répartition des pluies selon les mois: 8. ${ }^{\circ}$ Quantité d'eau tombée par jour de pluie : $90^{\circ}$ Groupement des pluies: $100^{\circ}$ Des vents de pluies. Da quanto abbiamo fin' ora rilevato in questi due sommi Uomini, si conchiude chiaramente, che non basta calcolare la quantità di pioggia annuale per un paese; ben vero la distribuzione della medesima in tutto il corso dell' anno, e specialmente di quello agronomico. Ma io vi diceva poc' anzi che due grandi conseguenze emanano dalle osservazioni meteorologiche del Sig. Grovene. Della prima vi ho fatto notare l'eco che ne fa il Sig. De Gasparin, gran Fisico e grande Agronomo, che citò l'opera, ma non l'Autore. Adesso vi fo osservare che il Sig. Grovene era sagacissimo, ed in ciò non voglio essere adulatore (Dio me ne guardi!) benvero voglio, e debbo stimare un tanto Uomo. Il Sig. Arciprete diceva che l' anno pluviale agronomico dovesse, almeno per la Regione Pugliese, cominciare dal settembre, e finire all' agosto dell' anno seguente. Diceva bene: ed io debbo confessare francamente che i suoi lumi, quelli dell' Abate Toaldo, quelli di De Gasparin, di De Candolle, e dell' interminabile e sommo Hunborot mi hanno talmente convinto, che ora sono più partigiano del fatto reale e positivo sotto l'occhio, anzichè del vago ed astratto. Mi spiego meglio. Il Sig. Abate diceva che fino all' agosto cominciano gli alberi ad entrare in nuovo succhio, ed a 
prepararsi, per cosi dire, ai frutti del nuovo anno. Noi dobbiamo afferrare, in queste espressioni, lo spirito scientifico dello Scrittore Molfettese, tutto allusivo alla fisiologia vegetale. Voi sapete bene che le gemme, e fiorifere, e foliifere, e miste, tutte si formano al tempo della discendenza de'succhi, i quali sono in movimento, tra il più ed il meno, nel mese di agosto presso di noi, considerando in massa tutta la vegetazione della parte centrale della zona temperata, e rispettando sempre le particolarità eccezionali delle varie stagioni e località. Ciò premesso, comecchè le gemme rappresentano il cominciamento della novella vita della pianta, la quale vita si farà più attiva nell' anno seguente, è facile il comprendere, che se le piogge non cadono a tempo in agosto o in settembre, le piante arboree nutriscono poco, e per di più soffrono, come il bambino che si abbia scarso e misero latte della sua madre. Conseguentemente ne risentirà la futura raccolta, poichè le piogge di agosto non servono pel solo frutto esistente in questo mese, ma anche per la pianta iutera. Ed un' altra pruova di questo fatto sta in ciò, che ordinariamente, salvi i casi eccezionali, ad una scarsa o nulla raccolta ne segue una copiosa, perchè la pianta sgravata del peso nel primo anno, meglio si nutrisce e fortifica per quello avvenire. Io pubblicai per le stampe un articolo sugli olivi, le di cui tenere frasche, rosicchiate dai vermini in un anno, produssero abbondante frutto in quello appresso, che ricompensò largamente la sofferta perdita. Leggetelo, perchè in esso trovasi a capello quanto su lo stesso fatto ne riferisce il nostro Abate, il quale si aveva 
circa quarant' anni di studio sugli olivi: e quarant' anni di studio pratico sono sufficienti per darne esatto giudizio. Continuando dunque a ragionare sulla formazione delle gemme ed altro, dico come siffatti principii fisiologici, che reggono costantemente in Natura, non così facilmente si gustano da tutti coloro che strisciano sulla superficialità de' giudizii ; quindi si teugono per falsi, sol perchè non si avverano sempre nei proprii campi, d'onde poi ne nasce il disprezzo e lo sbadiglio, ed in fine la diffidenza verso lo studio agrario! Ma passiamo avanti. Queste cose fisiologiche son ben conosciute. La mia veduta è tutt' altro, poichè io miro al positivo delle produzioni campestri ; e dico in poche parole che il Sig. Grovene aveva ragione, e diceva benissimo. $\mathbf{E}$ che a voler restringere in minimi termini la cosa; tutto si riduce, secondo me, alla seguente frase. L' anno pluviale agrovomico deve contarsi da

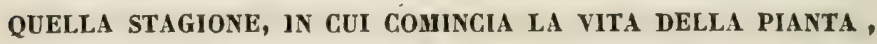
Giò̀ LA FORMaZione DELLE GEMMe PER LE ARBOREe, E LO AFFIDAMENTO AL TERRENO, 0 LO SBUCGIAR- SPONTANEO E NATUrale delle piante annuali. Questa regola, rigorosamente parlando, dovrebbe adottarsi per ogni specie di pianta, ed in questo modo audremmo all'infinito; ma in termini positivi e senza chimere devesi applicare alle diverse Regioni agrarie, perchè è cosa più facile e che meglio si avverte coi fatti patenti e manifesti. Voi, per esempio, sceglierete lo zafferano a coltivare: e bene l' anno pluviale agronomico per questa pianta, secondo me, dovrebbe contarsi da luglio a giugno, o da agosto a Juglio dell' anno appresso; affinchè il terreno si trovasse ricco di principii e 
succhi, già elaborati, e necessarii pel vegetabile nel tempo della piantagione de' suoi bulbi solidi, i quali, al dire del Sig. Cavalier Texone, lengono il dimezzo tra il vero bulbo ed il rizòma : per lo che io ne ho visto i fatti, che sono verissimi. Altri intanto direbbe che per questa pianta l' anno pluviale agronomico dovrebbe contarsi più presto; altri più tardi. M'importa poco. L'essenziale si è che il principio sommamente filosofico e generale a un tempo del Sig. Arciprete vuole che l' anno pluviale agronomico sia adattato e computato il più convenientemente possibile per la Regione agraria di uno o più paesi che fossero situati sotto una medesima zona climaterica e vegetativa. E quello indicato da lui è precisamente per la Puglia : ed io fo riverentemente eco alle sue filosofiche vedute; conciosiachè in Puglia l'acqua copiosa in agosto o in settembre è utilissima, sia che vogliate riguardarla per la vegetazione arborea degli alberi fruttiferi, sia per quella erbacea dei cereali, biade, e legumi, come quelli che ben preparato vogliono il terreno e fisicamente e chimicamente mediante il calorico, l' acqua, ed $\mathrm{i}$ lavori, in virtù de' quali elementi possa la Natura col tempo necessario operare nelle sue viscere e quindi renderlo adatto alla seminagione in ottobre: e ciò si otliene appunto quando piove abbondantemente in agosto o in settembre. Dai specchietti antecedenti, che avete visti distribuiti in cinque quadriennii, risulterebbe per media una quantità di pollici 19. 0.6. di pioggia all' anno, siccome lo stesso Sig. Abate dice, esprimendosi nei seguenti termini : 
Questa pioggia di pollici 19. o. $\frac{6}{2}$ cacle su di Molfetta in $7^{6}$ giorni per risultato medio in ciascun anno, e questi giorni piovosi sono cosi distribuiti:

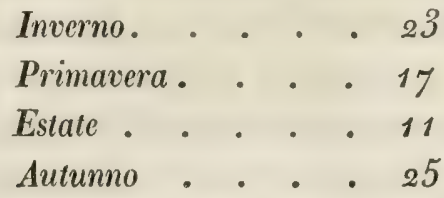

E poichè dividendosi 19. o. 6. per $7^{6}$ si hanno per quoziente tre lince, questa dee dirsi la somma media di un giorno piovoso, o, che è l' istesso, di una pioggia per Molfetta.

In venti anni di esatte osservazioni come si vede nella tavola che vi ho dimostrata, si rileva bene quanto dirò in seguito, sulla pioggia, dalla primavera all'autunno essere scarsa nella Peucezia tra Molfetta e Barletta. Di falti, sopra venti annate (dal 1784 al 1803) si contano sei marzi con poche linee di pioggia, e nel mese di marzo, si sa, che sempre l'acqua non manca; quindi non deve meravigliare se abbiamo sei marzi scarsi sopra venti. Vediamo il mese appresso, e troviamo nove aprili con poche linee di pioggia, de'quali nove, quello del 1803 diede perfetto zero. Seguono sette maggi con poche lince di acqua, dei quali uno presenta zero, e fu il 1796. Dodici giugni con poche linee, sopra venti, e fra essi, quello del 1802 portò zero. Di venti anni, sedici lugli con poche linee di pioggia, e di questi si contano tre collo zero, cioè il 1787, il 1790, ed il 1798. Dieci agosti sullo stesso piede, dei quali uno solo senza pioggia, e fu il 1802. Del settembre se ne contano dodici con poche linee di acqua. 
Ci troviamo ai conti, cioè che la state è quasi sempre sitibonda, cominciando progressivamente dalla decadenza della primavera fino al cominciamento dell' autunoo; tranne le annate eccezionali, in cui cadono parecchi pollici di pioggia, come accadde nel 1792; in cui i tre mesi di giugno, luglio, ed agosto diedero la pioggia cosi :

$$
\begin{array}{lrr}
\text { Giugno } & 2 . & 3.6 \\
\text { Luglio } & 2 . & 11.5 \\
\text { Agosto } & 6 . & 6.9 \\
\cline { 2 - 2 } & 11 . & 9.8
\end{array}
$$

Vale a dire undici pollici, nove linee, e otto dodicesimi. Lo che fu eccezionale per tutta la state. L'altra annata straordinaria in pioggia estiva fu il $\mathbf{1 8 0 0}$ che diede l' acqua, ma meno in quantità di quella del 1792. La pioggia vi cadde cosi distribuita:

$$
\begin{array}{llr}
\text { Giugno } & 2 . & 5.2 \\
\text { Luglio } & 3 . & 2.4 \\
\text { Agosto } & 3 . & 10.2
\end{array}
$$

\section{5. 8}

Cioè nove pollici, cinque linee, e otto dodicesimi. Or vedete, che in venti anni appena due stagioni estive piovose. Se vi fate a calcolare allo stesso modo la quantità della pioggia dall' autunno alla primavera, trovate presso a po- 
co lo stesso fenomeno; ma in senso inverso, cioè l'aumento progressivo e la diminuzione progressiva dell' acqua da una stagione all'altra, con qualche annata eccezionale secca nel verno. Cosi per esempio in venti anni abbiamo queste eccezioni : nel 1789 il mese di gennaio diede undici linee e quattro dodicesimi di acqua; nel 1796 il gennaio diede zero. Due gennai secchi sopra venti anni. Dei febbrari poi se ne contano settequasi asciutti in venti annate. E finalmente quattro dicembri scarsi a pioggia nello stesso periodo di tempo. Giova in ultimo avvertire che bilanciando tutte le venti annate fra loro, tranne qualche eccezione, si trova una tal quale compensazione tra il più ed il meno, che dà la media dei pollici 19 e 6 dodicesimi giusta i calcoli ed osservazioni del solerte nostro Abate. Ora possiamo accennare qualche cosetta sulla natura de'venti i più dominanti e che hanno maggiore influenza sulla vegetazione in massa delle campagne di Barletta. E servendomi della stessa espressione del nostro Scrittore, dirò dapprima che il vento Est è il ventipiovolo della Puglia Peucezia, ed anche apportator di neve. Compagni gli sono nella scarica della pioggia il Nord-Est: il vento Est-Nord-Est: il Sud-Est: ed il vento Est-Sud-Est Cosicchè volendosi calcolare esattamente le diverse gradazioni, nel maximum e nel minimum, dello stato igrometrico dell' atmosfera, converrebbe tener conto della maggiore o minore veemenza dei cinque suindicati venti che soffiassero nel momento delle osservazioni, o che avessero soffiato poco tempo innanzi, più o meno vaporosi o nuvolosi. Cosi, io dico, potrà l'osservatore in un giorno 
qualunque, nelle ore matutine, per esempio, notare il vento Nord-Nord-Ovest, il quale è secco e salutare sull' agro barulense, $\mathrm{e}$ intanto avvertirà qualche gradazione igrometrica nell' atmosfera: gradazione che si accrescerà nelle ore pomeridiane a misura che sarà cessato il Nord-Nord-Ovest, ed avrà preso piede il Sud-Est, siccome ordinariamente accade in quella plaga. Se egli dunque, l'osservatore, conoscerà che il vento del giorno antecedente sarà stato l'Est, o uno degli altri suoi quattro compagni, innanzi detti, renderà facilmente ragione a se stesso della causa produttrice dei vapori nell' atmosfera, i quali, oltre quelli provenienti dalle esalazioni terrestri del luogo, copiosamente vengono dalla regione orientale e marittima. Essi procedendo ai confini della Puglia, urtano contro gli Appennini; ivi si associano ad altri vapori, e ricalcitrando, addiventano più gravidi di prima, e apportano pioggia. I venti del Sud e del Sud-Ovest al contrario imperversando sulla Puglia, trovano una libera uscita sull' Adriatico, ed è facile che siano apportatori di pioggia sulle coste dell' isola di Corfù e su quelle di terraferma che l' è vicina. Il vento Sud-Est da se solo raramente apporta pioggia, ma se s'inclina un poco più all'Est, cangiasi in ventipiovolo. Vaporosi sono altresi il Sud e il Sud-Ovest, ma raramente piovosi. Benvero sono assai caldi, e di essi due a preferenza il Sud-Ovest, il quale quando soffia nella primavera indurisce di molto il terreno argilloso a danno delle piante erbacee ed in ispecie de'seminati di grano: se poi incalza nei mesi estivi, non solo dissecca maggiormente il terreno, ma danneggia e piante erbacee e piante arboree, di tal che 
gli alberi fruttiferi soffrono danni positivi, siccome vi accennai quando osservavamo la posizione topografica delle campagne barulensi, procedendo da Canosa a Barletta, se ben vi ricordate nella prima passeggiata. Ed io posso, e debbo assicurarvi che in un anno (non ricordo quale se nel 1841 o nel 1844) il vento Sud-Ovest fu così potente nel mese di luglio, che osservai, dopo parecchi giorni della sua sterminata influenza, in taluni vigneti non solo disseccati i pampani, i tralci, e i grappoli delle viti, ma anche buona parie del loro tronco, lo che fu doloroso senza dubbio a vedersi. Il vento Nord è potente assai, e la sua veemenza tanto maggiormente si avverte, in quanto che venendo dall'Adriatico, colpisce tutto a un tratto le camparne, non vi trovando ostacolo alcuno di boschi o di montagne, che ne polessero infrangere la forza. Si è al Nord, e precisamente quando il monte Gargàno è carico di neve che devesi il massimo abbassamento di temperatura nell' atmosfera barulense. Il vento Est e quello di Est-Nord-Est anche sono freddi, parlicolarmente nel verno, ma non raggiungono l'intensita prodotta dal Nord. Le nevi sono piuttosto rare, e nelle annate di abbondanza nevosa (che sono rarissime in un dato periodo di molti anni), esse non si elevano al di là di due palmi, e la loro durata è assai breve siccome mi ricordo sin dalla mia fanciullezza. Le gelate sono frequenti, ed accresciute nella loro intempestivita, per cosi dire, dalla vicinanza del monte Gargàno e del monte Vulture (e più dal primo), quando i venti spirano dalla direzione di uno di essi due, che fossero ancora coperti di neve nel mese di marzo in una qualche an- 
nata straordinaria. La grandine talvolta cade nella state, e con veemenza. Cade a zona più o meno larga, frequentando la direzione dall'Ovest all' Est, per quanto mi ricordo fin dalla tenera età. Sulla quale direzione dall'Ovest all' Est più frequente, io richiamo l' altenzione dei Fisici. I danni che la grandine apporta agli oliveti, ai vigneti, e ai mandorleti, sono gravissimi. La brina è frequente nel verno e talvolta in primavera; ed allora le seninagioni de'cereali e dei legumi, specialmente quelle de'secondi, risentono tristi effetti. La rugiada (corrottamente detta dai contadini acquàra) è copiosa e quasi perenne, dalla primavera all' autunno; ed è questa la ragione per cui, ad onta della secchezza del clima in generale, la vegetazione, eccettuata quella de'siti elevati ed estremamente calcari, non è tanto rattristata come se la potrebbe immaginare chi mai visitò siffatti luoghi. Io posso affermare sulla fede mia, che sin dalla età di anni dieci mi ricordo bene come i vigneti in settembre e in ottobre sono carichi di rugiada, le di cai goccioline copiose, sparse su pei grappoli e su i pampani, rappresentano tante piccole perie a traverso i raggi solari. Oltre a ciò, nel mese di maggio, quando ho traversate frequenti volte le praterie dell' Ofanto, ed i seminati di grano e di legumi per la raccolta delle piante spontanee, ne sono uscito sempre bagnato dai piedi sino al petto. Nel mese di giugno, luglio, ed agosto per le ricerche delle piante alla contrada delle paludi e a quella della fiumara, mi si bagnavano talmente le gambe, dalle sei sino alle nove del mattino, che spessu sono stato aggravato da forti rappigli e da altri malanni. E queste co- 
noscenze pratiche, da maggio ad agosto, poggiano sopra dieci anni di ricerche e di studio. Vi è dunque una parziale compensazione tra la copiosa rugiada e la forte evaporazione, di cui vi terrò parola da qui a poco. La nebbia vi è assai rara, ed appena nel periodo di parecchi anni se ne conta qualcuna piuttosto densa (lo che ha luogo nelle annate eccezionali): cosi nel 1840 , al mese di marzo, ne venne una dal mare, e propriamente dalla direzione di Nord-Est al Sud-Ovest, verso le ore 23 italiane; ed era cosi densa, che alla distanza di duecento palmi circa non vi si distinguevano più $i$ molti bastimenti mercantili che erano nella rada pel carico de' cereali. Invase le campagne, e verso le ore 24 italiane cominciò a dileguarsi. Nel giorno appresso vi fu cangiamento atmosferico, e dopo tre o quattro giorni cadde la pioggia. Ma, lo ripeto, questa meteora è rarissima. Vi ho parlato intanto più aranti del massino freddo: ora vi dirò del massimo caldo. La temperalura la più elevata proviene dal Sud e dal Sud-Ovest. E se a questo caldo si aggiunge la riverberazione bruciante dei terreni calcari, si vedrà quanto è forte l'influenza della temperatura elevata nei mesi estivi sulla regetazione delle piante coltivate e di quelle indigene.

Incipit ex illo montes Apulia notos

Ostentare mihi quos torret Atabulus.

Che se alla temperatura assai calda si associa la solita rarità delle piogge nei mesi estivi /Lingua siziente d'appu- 
la Cagna), si riconoscerà di leggieri a che soflerenza sono sottoposti i vegetabili. E tutle queste notizie sul clima barulense, ed inclusivamente della Peucezia, le trovate quasi del tutto identiche nella pregiatissima Memoria scrilta dal dottor D. Galiléo Palcotra sulla Puglia Dauaia; tranne qualche eccezionalità de' speciali siti. Se, per esempio, state precisamente sotto il Gargàno, o sopra monte $C a l$. vello, notate senza dubbio qualche particolarità d'influenza clintaterica alquanto diversa da quella che si avverte sull' Adriatico lungo il littorale tra Barletta e Molfetla. Intanto restringiamo quel poco che abbiamo fin' ora studiato sul clima che soprastà alle campagne di Barletta; e permettetemi che nel miglior modo approssimativo io ve lo formolassi nei seguenti termini:

Il clima delle campagne barulensi è assai ventilato, come quello degli altri paesi di Puglia, perchè non vi sono nè boschi, nè montagne. Dalla primavera all' autunno è piuttosto estremamente secco a tal segno, che nelle annate straordinarie (contatene sei sopra dieci) ben va detto per la state quanto segue:

Les cieux par Dieu fermés et devenus diairain, Et la terre trois mois sans pluie et sans rosée.

E se cadono piogge nei mesi caldi, la evaporazione è maś sima, si per l'influenza del vento, si per quella del sole, che per la nuncanza di boscaglie positive ed estese. L'arciprete Giovese ben dice quando si esprime con queste 
parole sulla distribuzione della pioggia in Puglia, durante il corso dell'anno. Quello, così egli, che vi è di male nella pioggia della Puglia si è, che sia mal distribuita, edirò bene se dirò che è distribuita all' inverso di quello che lo sia in Francia, e nel Nord dell' Europa. Il sig. DE GASPARiN quando tratta della evaporazione, dice delle cose brillanti; e noi meditiamo sopra diversi passi, uno de'quali è cosi concepito: Plus tard, quand nous aurons de bonnes séries d'observations de ce genre, nous ne doutons pas que l'on n'y trouve la solution d'une grande partie des problèmes que présentent les climats, car l'évaporation est aussi une synthèse qui combine les effets de la chaleur, de l'humidité de l'air, et de son agitation. E altrove dice. L'évaporation est un phénomène trop essentiel en agriculture pour être laissé dans l'oubli auquel le condamnent la plus part des traités de météorologie. Parlando poi della evaporazione della terra, dopo tanti belli calcoli ed osservazioni, soggiunge con questi detti. On voit avec quelle rapidité marche l'évaporation au début: le premier jour. quand la ter$r e$ est complétement imbibée, elle est de plus du quart de l'évaporation d'une surface aqueuse; elle diminue constamment; aprè̀s sept jours, elle n'est plus que le septième. Bientốt la terre se desséchant, elle devient presque inappréciab?e. Dès le deuxième jour, avec cette température $\left(230^{\circ}\right.$ à $24^{\circ}$ dans le mois d'Aoûl) la couche supéricure est sèche à la profondeur de 2 à 3 millimètres; au bout de huit jours, les plantes qui ne s'enfoncent pas à plus d'un décimètre commencent à souffirir, et réclument des arrosements. Questa è la vera condizione della Puglia. E quan- 
tunque vi è una cerla compensazione tra la evaporazione terrestre delle sue campagne e la quantità di rugiada che cade nella notte avanzata, da primavera ad autunno, ciò nondimeno conviene ricordarci quel tanto che emerge dalla meteorologia del sig. DE Gaspanin: cioè che non basta la quantità della pioggia in un paese, è necessaria del pari una tenue evaporazione. Mla in Puglia vi è assai evaporazione e poca pioggia, o per meglio dire col sig. GroVENE, vi è mal distribuita, ecco perchè si avvertono assai gli effelli del caldo e della siccita. Ed ancorchè la pioggia vi fosse ben distribuita, ad onta di ciò sempre la evaporazione ne porterebbe il primato. (L'évaporation l'emporterait sur la répartition de la pluie, direbbe un francese). Riprendendo adesso il filo della esposizione dei fatti sul riepilogo del quadro climaterico delle campagne barulensi, imprendo a dire che dall' autunno alla primavera il clima è mediocremente piovoso (quantunque spesso accada nel corso di lunghi anni, che in parecchie annate, strabocchevole sia la quantità dell' acqua per queste tre stagioni ), e la serenità talvolta vi è alquanto dominante; in maniera che, o la siccita è prolungata in autunno, ed allora s' incontrano difficollà per eseguire le seminagioni, o se sono già eseguite, non tutti i semi metton fuori loro germe egualmente, e nascono quindi piante stentate e grame: o la mancanza di pioggia si prolunga nel verno, ed in tal caso in taluni terreni sostanziosi i seminati cestiscono sotterra senza soffrire, ed in altri sterili cominciano a intisichire con foglie giallognole alle loro estremità. Nel verno il freddo è piuttosto assai sensibile, tanto per l'a- 
zione dei venti dominanti dell'Est e del Nord, quanto per la irradiazione notturna del calorico terrestre, la quale $\dot{\mathbf{e}}$ tanto più influente a danno dell' economia vegetale, in quanto che il cielo è soventi volte, in quella stagione, sereno, e poco o niente vaporoso. Benchè però è da ritenersi che le gelate, di breve durata, non sono sempre dannose da una parte, e dall'altra servono di lavoro nei terreni argillosi: ed è risaputo sin dagli antichi tempi che i loro effetti sono più o meno tristi secondo le località, le influenze de' venti, e l' azione del sole debole, o intensa. Il fenomeno in Puglia sulla irradiazione terrestre vorrebb'essere più direttamente studiato dai Fisici solto il rapporto della località in una Regione sufficientemente piana. Secondo le osservazioni scrupolose del nostro Autore sig. Giovene, il massinı abbassamento di temperatura giunge da $10^{\circ}$ sino a $7 .^{\circ}$ solto zero di Rénumur. Però il $7 .^{\circ}$ è rarissimo nelle annate di eccezione, come accadde tra il 1787 e 1788 , in cui le campagne, ed in particolarita gli olivi, furono sommamente danneggiate dal gelo; ed il lido di mare fu gremito di moltissimi e svariati pesci semivivi (cernie, denti$c i$, sarghi, seppie ec.), che galleggiando in ogni punto, furono tutti presi a sollievo de' poveri pescatori. Il sig. dottor Palcotra nella sua utile Memoria sulla Daunia riporta quanto segue: La massima fredda temperatura dell' Inverno è di $2 .^{\circ}$ e $30^{\circ}$ sotto zero del termometro di REAUMUR, ma ciò ha brevissima durata, nè poi si avvera in tutli gli ammi, anzi nel generale poche volle arriva a sostenersi a zero. Nella slate poi il caldu è eccessivo, e più volte soffocante, allorchè spirino i reuti del Sud e del 
Sud-Ovest. In venti anni di osservazioni termometriche il sig. Giovene ha trovato che il massimo caldo della state nella Peucezia, e specialmente a Molfetta, arriva dal 25. ${ }^{\circ}$ al 33. ${ }^{\circ}$ del Réaunur. (Egli in un anno nel mese di febbraro vide segnare $11 .^{\circ}$ all'ombra, mentre il sole batteva fuori l'ordinario: pose allora il termometro al sole, ed in un quarto d'ora segnò $34 .^{\circ} 1 / 2$ ). Il dottor D. Galileo Pallotra nella sua stessa suindicata Memoria sulla Puglia Daunia, parlando del gran caldo di quella Provincia, dice cosi: Il massimo calore della stagione estiva giunge ai gradi ventollo, al piu trenta, réaumuriani. Prima di por fine allo studio di queste utili conoscenze, permettetemi una osservazione. Il nostro Arciprete GroveNE facendo il paragone tra la quantità di pioggia annuale sopra Molfetta e quella di taluni altri paesi di Europa, e trovando che su di questi ne cade meno di quella che cade a Molfetta, imprende a difendere in qualche modo la Puglia, col dire che la quantita di pioggia in essa non è tanto scarsa, ma che solamente vi è mal distribuita, siccome abbiamo per lo innanzi visto. Però bisogna notare ed osservare che la evaporazione della Puglia è assai maggiore che non è quella de’ paesi di Europa, ove la quantità di pioggia annuale è minore di quella di Molfetta. Cosi per esempio il nostro Arciprete dice che a Parigi cadono anuualmente pollici 16: 1: 6 (badate bene che ora qui io mi servo della stessa misura adottata dall' esallo osservatore sig. Grovexe, in pollici, linee, e dodicesimi di lince, per non spezzare il filo delle idee secondo il suo linguagrio; ben inteso peró che la sudetta 
misura potremo ben ridurla a millimetri secondo il saggio e dotto sistema dei moderni Fisici e Meteorologisti) di pioggia, la quale è inferiore a quella di Molfetta rappresentata da pollici 19.0.6 : e da questo fatto vorrebbe dedurre non doversi considerare la Puglia tanto povera di pioggia quanto altri se la immaginasse. Noi facciamo notare che non basta mettere a calcolo la quantita di pioggia per un paese, ma conviene altresi studiare la sua distribuzione (come dottamente l'aveva studiata il nostro Arciprete e come lo ha detlato lo stesso DE GASPARIN) nel corso dell'anno e delle stagioni; più la evaporazione massima e minima; la vicinanza de' grandi bacini di acqua, cioè laghi, golfi, larghi fiumi ec.; le montagne, i boschi, i gradi di latitudine, quelli di elevazione dal livello del mare, ed altre svariate circostanze locali, che bisogna tutte calcolare. Cosi il sig. DE Gasparin su questo proposito riferisce quanto segue sulla distribuzione geografica delle piogge in Europa, e trova le medie seguenti :

millimetri

Italia al nord degli Appennini. 1136

Italia al sud degli Appennini, e nella Francia meridionale . . . . . . . .

Francia settentrionale e Alemagna. . . . 650

Questo fatto coincide bene con quanto diceva il sig. Grovene tra la quantità di pioggia che cade a Parigi e quella che cade a Molfetta, poichè Parigi si avvicina molto al Nord della Francia e Molfelta non dista gran trat- 
to dal Sud degli Appennini. Ma il nostro valente Arciprete dimenticava forse la grande evaporazione che ha luogo in Puglia sotto un Cielo puro, e la soverchia umidità del Nord della Francia, ove il Cielo è quasi sempre vaporoso e brumeux? E se aggiungiamo che Molfetta sta sul grado $41 .^{\circ}$ di latitudine e Parigi quasi all' estremità settentrionale del grado $48 .^{\circ}$, vedremo chiaramente che l'eccedente della pioggia di Molfetta, su quella di Parigi, non è gran fatto in favore della vegetazione riguardata dalla parte erbacea, ben vero dalla parte legnosa e conseguentemente arbustiva. Pare che basti fin quì, o Lettore, quel poco che abbiamo potuto studiare sulle campagne di Barletta e sul clima che lor vi domina. L'ora è avanzata, sono le due dopo mezza notte, e penso che anche voi al par di me sentiate il bisogno di riposare, dopo lo studio nei libri de'Crassicr. Domani muoveremo pel ritorno a Napoli; e perchè il cammino di quattro giorni (strada di ferro dove sei?!) non ci annojasse, noi percorreremo questo catalogo, che avete sott'occhio, di tutte le piante che io ho raccolte nell' agro barulense, un po la volta, nel periodo di anni dieci, contati e numerati, come per lo innanzi vi accennai, dal 1837 al 1847. Faremo le nostre osservazioni sopra quelle specie che crederemo le più rimarchevoli; ed ancorchè saremo per cadere in errore, noi prometteremo a noi stessi di rettificare il tutto con ulteriori studii che speriamo eseguire col tempo sopra luogo. Però, parlando delle campagne in generale, tanto sollo il rapporto botanico quanto solto quello agrario, per qual siasi paese del globo, cercheremo di scolpire nella 
nostra memoria le memorande parole di de Candolle sulle relazioni tra $\mathrm{i}$ limiti delle piante coltivate. Il arrive ici, egli dice, comme pour les plantes spontanées: les rélations entre les limites changent, suivant quion examine une certaine direction dans les plaines, ou certaines montagnes, plutôt que d'autres elc. ecc. Il serait aisé de multiplier ces exemples. La cause en est toujours dıns la variété des circostances de chaleur, froid, humidité, sécheresse, convenances locales agricoles, etc. qui influent sur les limites. C'est un avertissement pour ne pas juger facilement des climats par les cultures, ni du succès probable des cultures d'après certaines données sur les climats. (!!!) Cosi, or leggendo, or discutendo, ed ora osservando i fatti naturali che incontreremo lungo il cammino, noi giungeremo nella Capitale, ove, dopo avere ringraziata la grande amabilitả e compiacenza del celebre Botanico sig. cavaliere Grovanni Gus. SONE, per essersi degnato a indicarmi i nomi generici e specifici delle piante da me raccolte nelle campagne di mia patria Barletta, io potrò lietamente dire: essere stato sincero il mio impegno nell' avervi servitu per le due già eseguite perlustrazioni campestri, e assai gradita essernai stata la vostra amabile compagnia di che mi avete sommamente onorato. 


\section{ENUHERATIO PLANTARUII,}

QUAE IN AGRO BARULENSE SPONTE PROVENIUNT.

\section{Sectio I.}

PLANTAE DICOTYLEDONEAE

\section{ACA NTHACEAE}

Acanthus

spinosus

julio, augusto.

\section{AM ARANTHACEAE}

$\begin{array}{lll}\text { Amaranthus } & \text { prostratus } & \text { julio, septembri. } \\ & \text { hibridus } & \text { julio, septembri. } \\ & \text { sylvestris } & \text { julio, septembri. }\end{array}$

\section{A HENTACEAE}

Populus

alba martio, aprili. nigra martio, aprili. 
$-78-$

Salix

ULMUS

Villarsiana

martio, aprili.

purpurea

martio, aprili.

suberosa

martio, aprili.

campestris

martio, aprili.

AM Y GDALEAE

Pronus

spinosa

martio, aprili.

\section{APOCINEAE}

ViNGa

major

martio, majo.

\section{ASCLEPIADEAE}

Cynancou

aculum

julio, augusto.

\section{ASPERIFOLIAE}

Cerintae

aspera

marlio, aprili.

Cynoglossum

pictum

martio, majo.

cheirifolium

martio, aprili.

Echiux

plantagineum

aprili, majo.

calycinum

aprili, majo.

pyrenaicum

aprili, majo.

pustulatum

Heliotropiug europaeum

europaeum varietas majo, junio.

junio, octobri.

parvifolium junio, augusto. 


$\begin{array}{llc}\text { HeLrotropius } & \text { supinum } & \text { junio, augusto. } \\ \text { Lithospersum } & \text { tinctorium } & \text { aprili, majo. } \\ \text { Mrosotis } & \text { collina } & \text { aprili, majo. }\end{array}$

\section{BORAGINEAE}

Aschesa

hybrida

aprili, majo.

italica

aprili, majo.

Asperugo

procumbens

aprili, majo.

\section{CAMPANULACEAE}

Campanola erinus

Speculamia speculum

hybrida. aprili, majo. aprili, majo. aprili, majo.

\section{CAPPARIDEAE}

Capparis

sicula

junio, septembri.

\section{CAPRIFOLIACEAE}

LoNicera implexa

Sambucos

ebulus

majo, junio.

junio, julio.

\section{CARYOPHYLLEAE}

Alsine

rubra

media

junio, augusto.

junio, augusto. 
Alsine

Arenaria

Cerastium

Dianthus

Frankenia

Gypsophyea

LyCHNIS

Polycarpon

Sagina

Saponaria

Silene

\begin{tabular}{ll} 
& gallica \\
& bipartita \\
& rubella \\
& rubella varietas bra- \\
chyopetala & \multicolumn{1}{c}{ vespertina } \\
& vespertina varietas \\
& $\begin{array}{l}\text { conica canescens } \\
\text { muscipula } \\
\text { media }\end{array}$
\end{tabular}

majo, junio. majo, junio. martio, aprili. martio, aprili. aprili, majo. aprili, majo. junio, julio. julio, augusto. junio, septembri. majo, octobri. aprili, majo. majo, julio. martio, aprili. majo, augusto. majo, augusto. martio, aprili. aprili, majo. martio, majo. martio, aprili.

martio, aprili. aprili, majo. aprili, majo. aprili, majo. majo, julio. februario, majo. 


\section{CHENOPODIEAE}

\begin{tabular}{|c|c|c|}
\hline Atriplex & portulacoides & augusto, septembri. \\
\hline & diffusa & augusto, octobri. \\
\hline & polysperma & augusto, octobri. \\
\hline & prostrata & augusto, octobri. \\
\hline & macrodira & septembri, octobri. \\
\hline & triangularis & augusto, octobri. \\
\hline & Tornabeni & augusto, septembri. \\
\hline & angustifolia & septembri, octobri. \\
\hline & Halimus & augusto, octobri. \\
\hline ВЕTA & cicla & majo, septembri. \\
\hline & maritima & junio, septembri. \\
\hline Chenoponium & viride & julio, octobri. \\
\hline & murale & julio, octobri. \\
\hline & album & julio, oclobri. \\
\hline & vulvaria & julio, octobri. \\
\hline & opulifolium & julio, octobri. \\
\hline & fruticosum & junio, augusto. \\
\hline & ambrosioides & junio, octobri. \\
\hline & botrys & auguslo, octobri. \\
\hline & Jacquini & augusto, octobri. \\
\hline & urbicum & augusto, octobri. \\
\hline Saticonnia & herbacea & augusto, septembri. \\
\hline & fruticosa & augusto, septembri. \\
\hline & macrostachya & augusto, septembri. \\
\hline SALSOIA & Kali & julio, octobri. \\
\hline
\end{tabular}




$\begin{array}{ll}\text { Salsola soda } & \text { julio, octobri. } \\ & \text { tragus }\end{array}$

\section{CISTINEAE}

Heliantuenuea salicifolium

Barrelieri

asperum

\section{COMPOSIT AE}

\begin{tabular}{|c|c|c|}
\hline ANacyclus & tomentosus & majo, junio. \\
\hline \multirow[t]{5}{*}{ ANTIIEMIS } & tinctoria & julio, augusto. \\
\hline & maritima & junio, julio. \\
\hline & incrassata & marlio, majo. \\
\hline & cotula & majo, augusto. \\
\hline & mixta & aprili, majo. \\
\hline \multirow[t]{3}{*}{ Artemisia } & variabilis & septembri, octobri. \\
\hline & vulgaris & septembri, octobri. \\
\hline & caerulescens & augusto, septembri. \\
\hline Asterk & $\begin{array}{l}\text { Tripolium } \\
\text { acris }\end{array}$ & $\begin{array}{l}\text { augusto, septembri. } \\
\text { augusto, septembri. }\end{array}$ \\
\hline Atractylis & cancellata & majo, junio. \\
\hline Bellis & sylvestris & novembri, aprili. \\
\hline & perennis & novembri, aprili. \\
\hline & annua & novembri, majo. \\
\hline Bidens & tripartila & julio, octobri. \\
\hline Bupititalmui & spinosum & junio, augusto. \\
\hline Calendula & arvensis & deccmbri, marlio. \\
\hline
\end{tabular}

apriti, majo.

aprili, majo.

apriti, majo. 


\begin{tabular}{|c|c|c|}
\hline Canduncellus & $\begin{array}{l}\text { corymbosus } \\
\text { caeruleus }\end{array}$ & junio, julio.' \\
\hline \multirow[t]{5}{*}{ Cardudes } & nutans & junio, julio. \\
\hline & corymbosus & majo, julio. \\
\hline & pycnocephalus & majo, julio. \\
\hline & leucographus & majo, julio. \\
\hline & argyroa & majo, julıo. \\
\hline \multirow[t]{2}{*}{ Cardina } & lanata & julio, augusto. \\
\hline & gummifera & augusto, septembri. \\
\hline \multirow[t]{7}{*}{ Centaurea } & salmantica & majo, julio. \\
\hline & calcitrapa & majo, julio. \\
\hline & nicaensis & majo, julio. \\
\hline & solstizialis & majo, julio. \\
\hline & Crupina & majo, julio. \\
\hline & lappacea & majo, julio. \\
\hline & melitensis & majo, julio. \\
\hline \multirow[t]{3}{*}{ Chiondrilla } & juncea & augusto, septembri. \\
\hline & juncea varietas b. & augusto, septembri. \\
\hline & juncea varietas c. & augusto, septembri. \\
\hline \multirow[t]{3}{*}{ Cimpsanthenuy } & Myconis & marlio, majo. \\
\hline & segetum & martio, majo. \\
\hline & coronarium & aprili, majo. \\
\hline \multirow[t]{4}{*}{ Cichoriom } & intybus & junio, augusto. \\
\hline & intybus varietas b. & junio, augusto. \\
\hline & glabratum & junio, augusto. \\
\hline & divaricatum & junio, augusto. \\
\hline \multirow[t]{3}{*}{ Cricus } & pungens & julio, septembri. \\
\hline & lanceolatus & julio, septembri. \\
\hline & italicus & julio, septembri. \\
\hline
\end{tabular}


Cnicus

Conyza

Conepis

Cynara

Erigeron

Eupatoridu

Filago

GALACTITES

Hedxpyols

Helmintiia

Hieracium

Hyoseris

Hypochaeris

INULA

KentrophyLlư lanatum julio, septembri.

julio, augusto: julio, septembri. julio, augusto. majo, junio. martio, majo. junio, augusto. junio, septembri. julio, augusto. julio, augusto. julio, augusio. pyramidata pyramidata varietas spathulata tenuifolia julio, augusto. julio, augusto. junio, julio. majo, junio. junio, augusto. martio, majo. martio, majo. junio, septembri. junio, septembri. aprili, majo. martio, aprili. aprili, junio. aprili, junio. majo, junio. julio, octobri. julio, octobri. julio, septembri. 
Kentrophyleum turbinalum

julio, augusto.

Lactuca

LEONTODON

Matricaria

Onopordon

Picris

Pulicania

Rrigadiolus stellatus

stellatus varietas hebelaenus

edulis

Scolymus

Scorzonera

Senecio

Seriola

Silybun

Sonchus

saligna

saligna varietas $\mathbf{b}$.

Taraxacum

minimum

chamomilla

illyricum

spinulosa

dysenterica

hispanicus

maculatus

hirsuta

laciniata

decumbens

Columnae

vulgaris

erraticus

vernus

aetnensis

marianum

maritimus oleraceus

tenerrimus

tenerrimus varie-

tas b. aprili, majo.

julio, septembri.

julio, septembri.

februario, martio.

septembri, octobri.

martio, majo.

julio, augusto.

julio, septembri.

jutio, septembri.

aprili, majo.

aprili, majo.

aprili, majo.

junio, augusto.

junio, augusto.

aprili, majo.

aprili, majo.

aprili, majo.

aprili, majo.

decembri, aprili.

julio, septembri.

decembri, julio.

martio, junio.

junio, augusto.

julio, octobri.

martio, majo.

januario, majo. 


\section{$-86-$}

Sonchus

tenerrimus varie-

tas c. aprili, majo.

picrioides

Thinivcia

Tolpis

tuberosa

barbata

Tiragopogon undulatum

Tussilago

Urospermum

Xistmon

Farfara

picrioides

Dalechampii

strumarium

spinosum januario, majo. julio, octobri. majo, junio. aprili, majo. martio, aprili. majo, junio. majo, junio. julio, octobri. julio, octobri.

\section{CONYOLYULACEAE}

$\begin{array}{clc}\text { Convolvulus } & \text { sylvestris } & \text { junio, julio. } \\ & \text { arvensis } & \text { julio, octobri. } \\ & \text { tenuissimus } & \text { majo, julio. } \\ & \text { soldanella } & \text { julio, augusto. } \\ & \text { lucanus } & \text { aprili, junio. }\end{array}$

\section{CRASSULACEAE}

$\begin{array}{lll}\text { Cotveredon } & \text { umbilicus } & \text { majo, junio. } \\ \text { Crassula } & \text { Magnolii } & \text { aprili, majo. } \\ \text { Senum } & \text { stellatum } & \text { aprili, majo. } \\ & \text { glanduliferum } & \text { aprili, majo. } \\ & \text { galioides } & \text { junio, julio. } \\ \text { Slimpervivum } & \text { tectorum } & \text { junio, julio. }\end{array}$




\section{CRUCIFERAE}

\begin{tabular}{|c|c|c|}
\hline Alsssem & campestre & martio, aprili. \\
\hline Arabis & hirsula & marlio, aprili. \\
\hline Biscutelia & apula & aprili, majo. \\
\hline \multirow[t]{3}{*}{ Brissica } & campestris & februario, aprili. \\
\hline & fruticolosa & februario, junio. \\
\hline & eruca & aprili, majo. \\
\hline \multirow[t]{2}{*}{ Bunias } & erucago & martio, majo. \\
\hline & aspera & martio, majo. \\
\hline Cakile & maritima & junio, julio. \\
\hline Capselta & bursapastoris & januario, marlio. \\
\hline Cirdinine & hirsuta & febriario, martio. \\
\hline Cramibe & maritima & majo, junio. \\
\hline \multirow[t]{4}{*}{ Diplotaxis } & muralis & martio, aprili. \\
\hline & erucoides & martio, junio. \\
\hline & viminea & martio, junio. \\
\hline & tenuifolia & marlio, septembri. \\
\hline Draba & verna & januario, martio. \\
\hline Eruca & sativa & martio, majo. \\
\hline ISATIS & tinctoria & junio, julio. \\
\hline \multirow[t]{2}{*}{ LEPIDIUII } & draba & aprili, majo. \\
\hline & graminifolium & aprili, septembri. \\
\hline Moricandu & arvensis & martio, majo. \\
\hline Nastertidi & officinale & aprili, majo. \\
\hline NEsLia & paniculata & martio, majo. \\
\hline \multirow[t]{2}{*}{ Raphanus } & Raphanistrum & martio, aprili. \\
\hline & Landra & majo, julio. \\
\hline
\end{tabular}


$-88-$

Rapistaun

SENEBIERA

Sinapis

TillasPI

Sisvinarium

Bryonia

Ecroliuy

Cuscuta rugosum

paniculatum

coronopus

hispida

incana

dissecta

nigra

arvensis

alba

pubescens

irio

polyceration

vimineum

perfoliatum majo, julio.

majo, junio, majo, septembri.

martio, septembri. martio, majo. martio, majo. martio, majo. martio, majo. martio, majo. martio, majo. martio, majo. martio, majo. marlio, majo. aprili, junio.

\section{CUCURBITACEAE}

dioica

elaterium majo, junio.

junio, augusto.

\section{CUSCUTEAE}

epithymum

minor majo, julio.

majo, julio. 


\section{DAPHNOIDEAE}

$\begin{array}{lll}\text { Dapine } & \text { gnidium } & \text { majo, julio. } \\ \text { Passerina } & \text { pubescens } & \text { augusto, octobri. } \\ & \text { annua } & \text { julio, augusto. }\end{array}$

\section{DIPSACEAE}

Dipsacus

sylvestris

integrifolia

KnaUtia

Scabiosi

Cupani

grandiflora

grandiflora varictas

prolifera julio, augusto. julio, augusto.

aprili, majo.

julio, augusto.

junio, augusto.

\section{EUPHORBIACEAE}

Andracine

Croton

Euphoria telephioides

tinctorium

chamaesyce

peplis

exigua

peplus

terracina

paralias

falcata

pinea 


$\begin{array}{lll}\text { Euphonbia } & \begin{array}{l}\text { helioscopia } \\ \text { pubescens } \\ \text { ceratocarpa }\end{array} & \begin{array}{l}\text { februario, martio. } \\ \text { martio, novembri. } \\ \text { majo, augusto. }\end{array} \\ \text { Mercurialis } & \text { annua } & \text { decembri, majo. } \\ \text { Theligonum } & \text { Cynocrambe } & \text { majo, junio. }\end{array}$

\section{FUMARIACEAE}

Fumaria

$\begin{array}{ll}\begin{array}{l}\text { officinalis } \\ \text { officinalis varietas } \\ \text { micrantha }\end{array} & \text { martio, majo. majo. } \\ \text { Petteri } & \text { martio, majo. } \\ \text { leucantha } & \text { martio, majo. } \\ \text { agraria } & \text { martio, majo. } \\ \text { parviflora } & \text { martio, majo. }\end{array}$

\section{GENTIANEAE}

$\begin{array}{lll}\text { Crilora } & \text { intermedia } & \text { junio, julio. } \\ \text { Eryturaea } & \text { centaurium } & \text { majo, junio. } \\ & \text { tenuiflora } & \text { majo, augusto. } \\ & \text { spicata } & \text { majo, julio. }\end{array}$

\section{GERANIEAE}

Erodium

gruinum
chium
moschatum
cyconium

aprili, majo. februario, majo. februario, majo. februario, majo. 
$-91-$

$\begin{array}{lll}\text { ERodium } & \text { malachoides } & \text { februario, majo. } \\ & \text { Botrys } & \text { februario, majo. } \\ & \text { cicutarium } & \text { martio, majo. } \\ & \text { cicutarium varietas } & \text { martio, majo. } \\ \text { Geranion } & \text { rotundifolium } & \text { martio, aprili. } \\ & \text { molle } & \text { martio, aprili. }\end{array}$

\section{HEDERACEAE}

ViTis

vinifera sylvestris majo,junio.

\section{HYPERICINEAE}

HYPERICUM

crispum

julio, septembri.

perforatum

majo, julio.

\section{JASPIPEAE}

Ligustruar

vulgare

aprili, majo.

\section{LABIATAE}

$\begin{array}{lll}\text { Ajuga } & \text { chia } & \text { aprili, majo. } \\ \text { Ballota } & \text { faetida } & \text { majo, septembri. } \\ \text { Lamion } & \text { amplexicaule } & \text { marlio, aprili. } \\ & \text { purpureum } & \text { martio, aprili. } \\ \text { Lycopos } & \text { europaeus } & \text { augusto, octobri. } \\ \text { Marnobium } & \text { apulum } & \text { junio, julio. } \\ & \text { Alysson } & \text { majo, junio. }\end{array}$


Mentila

Pinlonis

SALVIA

Satureja

Sideritis

StaciIys

Tedgrium

Trnvuus pulegium

sylvestris

hirsuta

aquatica

polystachya

macrostachya

macrostachya va-

rielas julio, septembri.

herbaventi

Verbenaca

viridis

clandestina

argentea

graeca

romana

salviaefolia

chamaedris

scordioides

pseudo-hyssopus

Zygis

Acynos

nepeta

nepeta varielas

capitatus julio, septembri.

julio, septembri.

julio, septembri.

julio, septembri.

julio, septembri.

julio, septembri.

majo, junio.

martio, junio.

martio, majo.

martio, majo.

junio, julio.

majo, augusto.

majo, junio.

junio, julio.

junio, julio.

majo, junio.

julio, septembri.

majo, junio.

majo, junio.

majo, octobri.

martio, majo.

junio, augusto. 


\section{LINEAE}

LinuM

$\begin{array}{lc}\text { gallicum } & \text { aprili, majo: } \\ \text { strictum } & \text { aprili, majo. } \\ \text { austriacum } & \text { aprili, majo. } \\ \text { corymbulosum } & \text { aprili, majo. } \\ \text { angustifolium } & \text { aprili, majo. }\end{array}$

\section{LYTHRARIEAE}

Lytimuir

salicaria

junio, septembri.

Graefferi

junio, septembri.

hyssopifolium

junio, septembri.

dibracteatum

junio, septembri.

\section{LORA PU⿴囗十⺝A CEAE}

Viscun

album

januario, februario.

MALVACEAE

Altinaea

officinalis

julio, augusto.

cannabina

junio, julio.

Hrisiscus

Trionum

augusto, septembri.

Lavatera

neapolitana

punctata

martio, majo.

junio, julio.

MaLope

Malachoides

Malva

parviflora

majo, junio.

martio, majo. 
$-94-$

Malva

nicaensis

sylvestris

vulgaris

martio, majo.

martio, majo.

martio, majo.

\section{MOREAE}

$\begin{array}{lll}\text { Caprificus } & \text { rugosa? } & \text { junio, julio. } \\ & \text { oblongata? } & \text { junio, julio. } \\ & \text { pedunculata? } & \text { junio, julio. } \\ & \text { sphaerocarpa? } & \text { junio, julio. } \\ \text { Ficus } & \text { carica sylvatica } & \text { junio, septembri. }\end{array}$

\section{OLEACEAE}

$\begin{array}{lll}\text { Fraxinus } & \text { excelsior } & \text { martio, aprili. } \\ & \text { rostrata } & \text { martio, aprili. } \\ \text { Orea } & \text { parvillora } & \text { martio, aprili. } \\ \text { Pmillyraea } & \text { europaea oleaster } & \text { majo, jumio. } \\ & \text { angustifolia } & \text { aprili, majo. } \\ & \text { media } & \text { aprili, majo. } \\ & \text { media varietas } & \text { aprili, majo. }\end{array}$

\section{O NAGRACEAE}

$\begin{array}{lll}\text { Callitriche } & \text { verna } & \text { majo, julio. } \\ \text { Epilobium } & \text { hirsutum } & \text { junio, augusto. }\end{array}$




\section{OROBA N CHEAE}

Orobanche

$$
\begin{aligned}
& \text { pruinosa aprili, majo. } \\
& \text { - . . ? aprili, majo. } \\
& \text {. . . ? aprili, majo. }
\end{aligned}
$$

\section{OXALIDEAE}

Oxalis corniculata martio, septembri.

\begin{tabular}{|c|c|c|}
\hline Anagrnis & faetida & januario, martio. \\
\hline \multirow[t]{2}{*}{ Astragalos } & sesameus & aprili, majo. \\
\hline & hamosus & aprili, majo. \\
\hline GLYCYRRHIZA & glabra & junio, augusto. \\
\hline Hedysarum & echinatum & aprili, majo. \\
\hline
\end{tabular}

$\begin{array}{lll}\text { Gladcion } & \text { luteum } & \text { majo, augusto. } \\ \text { PaPaver } & \text { siculum } & \text { aprili, majo. } \\ & \text { bybridum } & \text { aprili, majo. } \\ & \text { dubium } & \text { aprili, majo. } \\ & \text { Rhoeas } & \text { aprili, majo. }\end{array}$

\section{PAPILIONACEAE}


Hippociepis

LATHYRUS

Lotus

Medicago unisiliquosa

Aphaca

annuus

Ochrus

edulis

cylisoides

decumbens

decumbens varietas

corniculatus

corniculatus varie-

tas nanus

augustissimus

ornithopodioides

rectus

hirsulus

siliquosus

lupulina

circinata

scutellata

lappacea

lappacea varictas

paucigyrosa

Histrix

terebellum

minima

recta

maculata

sphoerocarpa

tuberculata aprili, majo.

aprili, majo.

aprili, majo.

aprili, majo.

martio, aprili.

junio, augusto.

junio, augusto.

junio, augusto.

junio, augusto.

junio, julio.

junio, augusto.

majo, junio.

majo, junio.

majo, junio.

majo, junio,

aprili, majo.

aprili, majo.

aprili, majo.

aprili, majo.

aprili, majo.

aprili, majo.

martio, majo.

aprili, majo.

aprili, majo.

aprili, majo.

aprili, majo.

aprili, majo. 
Mrdicago

Melilotus

ONONIS

Onomnychis

Ornituropus

Psonalea litoralis

litoralisvarietaslon-

orbicularis

orbicularis varietas

aprili, majo.

giseta aprili, majo.

applanata aprili, majo.

mollissima

aprili, majo.

agrestis

murex

denticulata

marina

coronata

falcata

falcata varietas ver-

sicolor majo, augusto.

falcata varietas ma-

jor majo, augusto.

leucantha

longifolia

parvillora

sulcata

variegata

brevillora

diffusa

spinosa

caput-galli

scorpioides

compressus

biluminosa aprili, majo.

aprili, majo.

aprili, majo.

aprili, majo.

majo, julio.

aprili, majo.

majo, augusto.

junio, julio.

aprili, majo.

aprili, majo.

aprili, majo.

aprili, majo.

aprili, majo.

aprili, majo.

julio, septembri.

aprili, majo.

aprili, majo.

aprili, majo.

aprili, julio. 
$-98-$

Sconpiunus

Thifolium

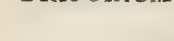

Thigonela

Vicia aprili, majo.

aprili, majo.

aprili, majo.

aprili, majo.

aprili, majo.

aprili, majo.

aprili, majo.

aprili, majo.

aprili, majo.

aprili, majo.

aprili, majo.

aprili, majo.

aprili, majo.

aprili, majo.

aprili, majo.

aprili, majo.

aprili, majo.

aprili, majo.

martio, aprili.

martio, aprili.

martio, aprili.

aprili, majo.

aprili, majo.

aprili, majo.

aprili, majo.

aprili, majo.

aprili, majo.

aprili, majo.

aprili, majo. 


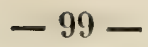

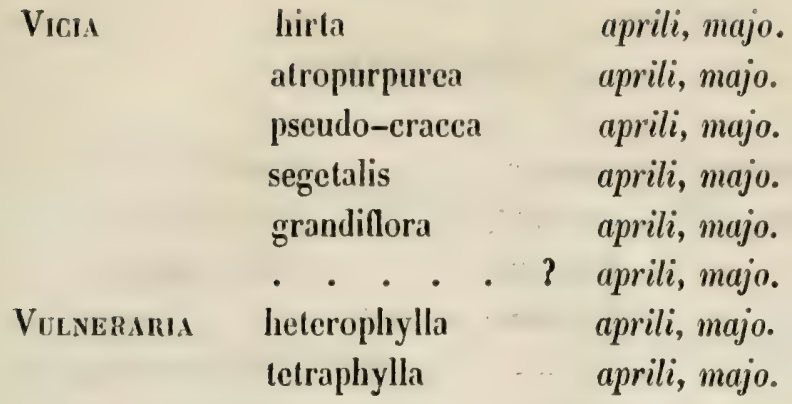

\section{PARONYCHIEAE}

Hernaria cinerea majo, julio.

\section{PLANTAGINEAE}

$\begin{array}{lll}\text { Plastago } & \begin{array}{l}\text { major } \\ \text { lanceolata }\end{array} & \begin{array}{l}\text { majo, septembri. } \\ \text { aprili, majo. }\end{array} \\ & \begin{array}{r}\text { lanceolata varietas } \\ \text { spicis capitatis }\end{array} & \text { majo, augusto. } \\ \text { lanceolata varietas } & \\ & \text { altissima } & \text { majo, julio. } \\ \text { Imaritima } & \text { julio, septembri. } \\ \text { serraria } & \text { majo, junio. } \\ \text { coronopus } & \text { majo, junio. } \\ \text { eriostachya } & \text { majo, junio. } \\ \text { commutata } & \text { majo, junio. } \\ \text { lagopus } & \text { majo, junio. } \\ \text { lagopus varietas } & \text { majo, junio. } \\ \text { albicans } & \text { aprili, majo. }\end{array}$




\section{PLUMBAGINEAE}

$\begin{array}{llll}\text { Plumbago } & \begin{array}{l}\text { europaea } \\ \text { Statice }\end{array} & \begin{array}{l}\text { julio, octobri. } \\ \text { julio, octobri. } \\ \text { limonium } \\ \text { limonium }\end{array} & \begin{array}{l}\text { julio, octobri. } \\ \text { varietas } \\ \text { minor }\end{array} \\ & \text { julio, octobri. } \\ \text { caspia } & \text { junio, julio. } \\ \text { dubia } & \text { julio, octobri. } \\ & . & . & \text { majo, junio. }\end{array}$

\section{POLYGONEAE}

$\begin{array}{lll}\text { Enex } & \text { spinosa } & \text { aprili, junio. } \\ \text { Polygonus } & \text { maritimum } & \text { junio, augusto. } \\ & \text { lepathifolium } & \text { junio, augusto. } \\ \text { incanum } & \text { junio, augusto. } \\ & \text { serotinum } & \text { junio, augusto. } \\ \text { tenuifolium } & \text { junio, augusto. } \\ \text { aviculare } & \text { junio, augusto. } \\ & \text { Bellardi } & \text { junio, augusto. } \\ & \text { convolvulus } & \text { julio, augusto. } \\ & \text { bucephalophorus } & \text { martio, aprili. } \\ \text { Rumex } & \text { pulcher } & \text { majo, junio. } \\ & \text { pseudo-acetosa } & \text { majo, junio. } \\ & \text { hydrolapathum } & \text { junio, julio. } \\ & \text { elongatus? } & \text { junio, julio. } \\ & \text { conglomeratus } & \text { majo, junio. }\end{array}$


Rumex

patientia

majo, junio.

thyrsoides

aprili, majo.

\section{POMACEAE}

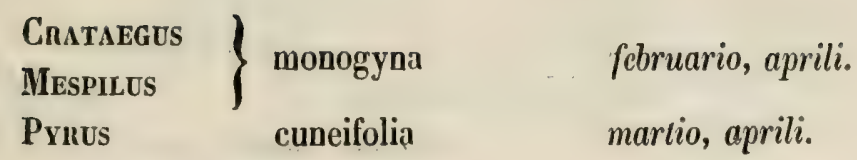

PORTULACEAE

Pontulaca oleracea majo, septembri.

\section{PRIMULACEAE}

$\begin{array}{lll}\text { Anagallis } & \text { arvensis } & \text { martio, majo. } \\ \text { Sanolus } & \text { valerandi } & \text { majo, julio. }\end{array}$

\section{RHAMPEAE}

$\begin{array}{lll}\text { Paliunus } & \text { vulgaris } & \text { martio, majo. } \\ \text { Riiamnos } & \text { alaternus } & \text { februario, martio. } \\ \text { Zyzypios } & \text { vulgaris } & \text { majo, junio. }\end{array}$

\section{RANUNCULACEAE}

Adonis

cupaniana

martio, aprili.

ANEMONE

hortensis

februario, aprili. 


\section{$-102-$}

A NEMONE

Clemitis

Delpinnium

Ficaria

Nigella

Rinunculus bullatus

sceleralus

'́luslictrum nigricans

\section{RESEDAGEAE}

Reseda philonotis

muricatus

flore albo februario, aprili. februario, aprili. coronaria varietas

flore albo februario, aprili. majo, julio. majo, julio. varietas fragrans majo, julio. junio, julio. januario, martio. martio, aprili. junio, augusto. octobri, novembri. martio, julio. martio, julio. martio, julio. aprili, majo. luteola

alba

fruticulosa

virescens majo, julio. majo, julio. februario, novembri. majo, julio. 
ROSACEAE

$\begin{array}{lll}\text { Potentilda } & \text { replans } & \text { majo, junio. } \\ \text { Poteriun } & \text { garganicum } & \text { aprili, junio. } \\ \text { Rosi } & \text { sempervirens } & \text { aprili, junio. } \\ & \text { dumetorum } & \text { aprili, junio. } \\ & \text { canina } & \text { aprili, junio. } \\ \text { Robus } & \text { dalmalicus } & \text { majo, septembri. }\end{array}$

\section{RUBIACEAE}

\begin{tabular}{|c|c|c|}
\hline Aspertula & arvensis & aprili, majo. \\
\hline & laevigata & aprili, majo. \\
\hline & cynanchica & julio, septembri. \\
\hline Galium & verum & junio, julio. \\
\hline & aparine & martio, majo. \\
\hline & saccharatum & martio, majo. \\
\hline & mollugo & majo, julio. \\
\hline & murale & junio, julio. \\
\hline Rubra & Bocconi & aprili, junio. \\
\hline & peregrina & aprili, junio. \\
\hline Sherardia & arvensis & aprili, majo. \\
\hline VALANTIA & muralis & aprili, majo. \\
\hline
\end{tabular}




\section{RUTACEAE}

$\begin{array}{lll}\text { Hypecodar } & \text { procumbens } & \text { februario, aprili. } \\ \text { Peganum } & \text { harmala } & \text { majo, junio. } \\ \text { Ruta } & \text { bracteosa? } & \text { majo, julio. } \\ \text { Trmudus } & \text { terrestris } & \text { majo, octobri. }\end{array}$

\section{SANTALACEAE}

$\begin{array}{lll}\text { Osyris } & \text { alba } & \text { majo, junio. } \\ \text { Tiesıum } & \text { humile } & \text { aprili, majo. }\end{array}$

\section{SAXIFRAGEAE}

Saxifraga

tridactylites

aprili, majo.

\section{SCROPHULARINEAE}

\begin{tabular}{|c|c|c|}
\hline \multirow{4}{*}{$\begin{array}{l}\text { AntirRmindi } \\
\text { Buntsia }\end{array}$} & Oronlium & augusto, septembri \\
\hline & trixago & aprili, majo, \\
\hline & $\begin{array}{r}\text { trixago varietas ver- } \\
\text { sicolor }\end{array}$ & aprili, majo. \\
\hline & viscosa & martio, majo. \\
\hline \multirow[t]{5}{*}{ Linaria } & cymbalaria & aprili, augusto. \\
\hline & graeca & julio, augusto. \\
\hline & triphylla & martio, majo. \\
\hline & spuria & julio, septembri. \\
\hline & simplex & aprili, majo. \\
\hline
\end{tabular}




\begin{tabular}{|c|c|c|}
\hline IINARIa & vulgaris & augusto, octobri. \\
\hline & vulgaris varietas & \\
\hline & speciosa & augusto, octobri. \\
\hline & minor & junio, julio. \\
\hline VERBASCUM & thapsus & junio, julio. \\
\hline & sinuatum & junio, septembri. \\
\hline & repandum & majo, julio. \\
\hline Veronica & cymbalaria & februario, martio. \\
\hline & Buxbaumii & februario, martio. \\
\hline & didyma & februario, martio. \\
\hline & arvensis & februario, martio. \\
\hline & beccabunga & junio, septembri. \\
\hline & anagallis & junio, septembri. \\
\hline & anagalloides & junio, septembri. \\
\hline
\end{tabular}

\section{SMILACEAE}

Suilax

aspera

septembri, octobri.

\section{SOLANACEAE}

$\begin{array}{lll}\text { Datura } & \text { stramonium } & \text { julio, septembri. } \\ \text { Hyosciamus } & \text { albus } & \text { februario, decembri. } \\ & \text { niger? } & \text { majo, junio. } \\ \text { Lycium } & \text { europaeum } & \text { julio, septembri. } \\ \text { Soldand } & \text { nigrum } & \text { majo, augusto. } \\ & \text { miniatum } & \text { majo, augusto. }\end{array}$




\section{TAMARISCINEAE}

Tamarix

gallica

junio, septembri.

africana

junio, septembri.

\section{TEREBINTHACEAE}

$\begin{array}{lll}\text { Prstacia } & \text { Lentiscus } & \text { martio, majo. } \\ \text { Ruos } & \text { coriaria } & \text { junio, angusto. }\end{array}$

\section{UMBELLIFERAE}

Anvi

majus

junio, julio.

glaucifolium

julio, septembri.

ApIUn

graveolens

julio, augusto.

Bifora

flosculosa

aprili, majo.

Bupleurum

protractum

aprili, junio.

Columnae

septembri, octobri.

Cacinrys

pungens

Caugalis

maritima

Conium

maculatum

majo, junio.

junio, julio.

junio, julio.

Coniandrum

sativum

testiculatum

Cruthumain

naritimum

aprili, majo.

aprili, majo.

julio, septembri.

Daucús

carota

aprili, majo.

polygamus

januario, martio.

polygamus varietas

floribus centrali-

bus rubris aprili, julio. 


\begin{tabular}{|c|c|c|}
\hline Dadcus & polygamus varietas & \\
\hline & proliferus & junio, augusio. \\
\hline EGHiNophora & spinosa & julio, octobri. \\
\hline & tenuifolia & augusto, septembri. \\
\hline EnYNGIUy & campestre & julio, septembri. \\
\hline & maritimum & julio, septembri. \\
\hline Ferula & communis & aprili, majo. \\
\hline Foeniculem & yulgare & junio, augusto. \\
\hline & piperatum & junio, augusto. \\
\hline Oenantue & pimpinelloides & aprili, junio. \\
\hline Opopanax & chironium & majo, junio. \\
\hline Pтусотніs & verticillata & majo, junio. \\
\hline SGandix & pecten & martio, aprili. \\
\hline Sidm & stoloniferum & junio, augusto. \\
\hline & intermedium & junio, augusto. \\
\hline & incisum & junio, augusto. \\
\hline Tordyliun & apulum & aprili, majo. \\
\hline Toritis & helvetica & aprili, junio. \\
\hline & nodosa & aprili, junio. \\
\hline & purpurea & aprili, junio. \\
\hline
\end{tabular}

\section{URTICACEAE}

$\begin{array}{lll}\text { Parietaria } & \text { erecta } & \text { majo, julio. } \\ & \text { diffusa } & \text { decembri, aprili. } \\ & \text { lusitanica } & \text { februario, martio. } \\ \text { Urtica } & \text { urens } & \text { novembri, majo. } \\ & \text { membranacea } & \text { novembri, majo. } \\ & \text { balearica } & \text { novembri, majo. }\end{array}$




\section{VALERIANEAE}

VALERIANELra echinata

eriocarpa

eriocarpa

bracteis uncinatis

auricula

dentata

pumila

\section{VERBENACEAE}

Verbena

ViteX

Viola officinalis

supina

agnuscastus

agnuscastus varietas

flore roseo

\section{VIOLARIEAE}

arvensis martio, aprili.

martio, apriti.

martio, aprili. majo, junio. majo, junio. majo, junio.

majo, septembri. julio, septembri: julio, septembri.

julio, septembri.

aprili, majo. 


\section{Sectio II.}

PLANTAE MONOCOTYLEDONEAE

\section{ALISMACEAE}

Alisma

plantago

junio, augusto.

\section{AMARYLLIDEAE}

Pancration maritimum

julio, augusto.

Sternbergia

Amaryllis $\}$ lutea

septembri, octobri.

\section{AROIDEAE}

$\begin{array}{lll}\text { Arisarur } & \text { vulgare } & \text { februario, martio. } \\ \text { Ardm } & \text { italicum } & \text { aprili, majo. } \\ & \text { tenuifolium } & \text { junio, septembri. }\end{array}$

\section{CYPEROIDEAE}

Carex

muricata

majo, junio.

divisa

majo, junio.

maxima

majo, junio. 


$\begin{array}{lll}\text { Cladium } & \text { germanicum } & \text { majo, septembri. } \\ \text { Cyperus } & \text { olivaris } & \text { julio, octobri. } \\ & \text { fuscus } & \text { julio, octobri. } \\ & \text { mucronatus } & \text { julio, octobri. } \\ & \text { tenuiflorus } & \text { junio, septembri. } \\ & \text { badius } & \text { junio, septembri. } \\ & \text { myriostachys } & \text { julio, septembri. } \\ & \text { junciformis } & \text { julio, septembri. } \\ & \text { palustris } & \text { junio, octobri. } \\ \text { Scinpus } & \text { maritimus } & \text { julio, septembri. } \\ & \text { holoschoenus } & \text { julio, septembri. }\end{array}$

\section{COECHICACEAE}

Colchucum Cupani

\section{GRAMINEAE}

\begin{tabular}{|c|c|c|}
\hline AEgYlops & ovata & aprili, majo. \\
\hline \multirow[t]{3}{*}{ Agrostis } & alba & aprili, majo. \\
\hline & pungens & aprili, majo. \\
\hline & verticillata & aprili, majo. \\
\hline AIra & capillaris & aprili, majo. \\
\hline Alopecuros & agrestis & aprili, majo. \\
\hline ANDROPOGON & pubescens & aprili, majo. \\
\hline ANTHOXANTHUM & odoratum & majo, junio. \\
\hline Arundo & dopax & augusto, octobri. \\
\hline Avena & stterilis & aprili, majo. \\
\hline & strigosa & aprili, majo. \\
\hline
\end{tabular}




\begin{tabular}{|c|c|c|}
\hline Avena & fragilis & aprili, majo. \\
\hline \multirow[t]{2}{*}{ Briza } & maxima & aprili, majo. \\
\hline & minor & aprili, majo. \\
\hline \multirow[t]{4}{*}{ Bromus } & pinnatus & majo, junio. \\
\hline & madritensis & aprili, majo. \\
\hline & mollis & aprili, majo. \\
\hline & mollis varietas & aprili, majo: \\
\hline Catapodiun & loliaceum & aprili, majo. \\
\hline Chrysurtis & cynosuroides & aprili, majo. \\
\hline Crupsis & alopecuroides & julio, augusio. \\
\hline Crnodon & dactylon & majo, augusto. \\
\hline \multirow[t]{2}{*}{ Cynosurus } & echinatus & aprili, majo. \\
\hline & giganteus & aprili, majo. \\
\hline Dactylis & glaucescens & aprili, majo. \\
\hline Eragrostis & megastachya & julio, augusto. \\
\hline \multirow[t]{3}{*}{ Festuca } & elatior & aprili, junio. \\
\hline & ligustica & aprili, majo. \\
\hline & ciliata & aprili, majo. \\
\hline GLyceria & fluitans & junio, augusto. \\
\hline HoLchus & lanatus & aprili, majo. \\
\hline HoRdeum & murinum & aprili, majo. \\
\hline \multirow[t]{4}{*}{ KoELERIA } & phleoides & aprili, majo. \\
\hline & cristata & aprili, majo. \\
\hline & villosa & aprili, majo. \\
\hline & $\begin{array}{l}\text { villosa varietas cul- } \\
\text { mis longe exertis }\end{array}$ & aprili, majo. \\
\hline Lagurus & ovatus & aprili, majo. \\
\hline LAPPAGO & racemosa & julio, auguslo. \\
\hline LOLIUM & perenne & majo, augusto. \\
\hline
\end{tabular}


LOLIUM

Milium

Panicum

Phalaris

Phleum

Phraguites

PoA

Polypogon

Rotboelea

SCLEROCHLOA

Secale

Setaria

Sphenopus

STIPA perenue varietas

maximum

multiflorum

arvense

tenue

temulentum

multiflorum

sanguinale

crusgalli

minor

paradoxa

tenue

communis

rigida

annua

bulbosa

trivialis

monspeliense

cylindrica

filiformis

maritima

maritima varietas

cereale

verticillata

viridis

glauca

divaricatus

pennata

tortilis majo, augusto.

aprili, majo.

aprili, majo.

aprili, majo:

aprili, majo.

aprili, majo.

junio, julio.

julio, augusto.

junio, augusto.

aprili, majo.

majo, julio.

majo, julio.

augusto, octobri.

aprili, majo.

aprili, majo.

aprili, majo.

aprili, majo.

majo, junio.

junio, julio.

junio, julio.

aprili, majo.

aprili, majo.

aprili, majo.

junio, augusto.

junio, augusto.

julio, augusto.

aprili, majo:

aprili, junio.

aprili, majo. 


\section{Triticom}

Gladiolus

IRIS

Romulea

$\begin{aligned} & \text { scirpeum } \\ & \text { repens } \\ & \text { repens varietas Le- } \\ & \text { ersianum }\end{aligned}$
villosum
lolioides
junceum
acutum

junio, julio.

aprili, majo.

junio, julio. aprili, majo. aprili, majo. junio, julio. junio, julio.

\section{IRIDEAE}

$\begin{array}{ll} & \text { sisyrinchium } \\ & \text { pseudoacorus } \\ \text { RoMULEA } & \text { bulbocodium } \\ & \text { ramiflora }\end{array}$

\section{JUNCACEAE}

Juncus

acutus
maritimus
rigidus
multiflorus
lampocarpus
articulatus
acutiflorus
Gerardi
insularis

junio, septembri. junio, septembri. junio, septembri. junio, septembri. junio, septembri. junio, septembri. junio, septemơri. junio, septembri. junio, septembri. 
Juncus

Alluum

Asparagus

Asphodelus

Hyacinthus

Muscari

Ornithogaldu
Gagea

commutatum

neglectum

botryoides

umbellatum

umbellata

villosum

villosum

varietas

floribus odoris

tenuifolium

Scilla

maritima junio, septembri. junio, septembri. junio, septembri.

majo, junio.

majo, junio.

majo, junio.

iunio, julio.

junio, julio.

augusto, septembri.

augusto, septembri.

majo, junio.

martio, aprili.

martio, aprili.

aprili, majo.

martio, aprili.

martio, aprili.

martio, aprili.

februario, martio.

februario, martio.

martio, aprili.

martio, aprili.

martio, aprili.

martio, aprili.

aprili, majo.

julio, septembri. 
$-115-$

$\begin{array}{lll}\text { ScILLA } & \begin{array}{l}\text { autumnalis } \\ \text { undulata? }\end{array} & \text { septembri, octobri. } \\ \text { Tulipa } & \begin{array}{l}\text { sylvestris } \\ \text { apula }\end{array} & \text { martio, aprili. } \\ & \text { martio, aprili. }\end{array}$

NAJADEAE

Lenna

gibba

trisulca

minor

Potamogeton

ZanNichellia pectinatum

palustris junio, augusto.

junio, augusto.

junio, augusto.

junio, augusto.

majo, junio.

\section{ORCHIDEAE}

Hrianthoglossem hircinum

Ophrys

Orchus lutea

arachnites

moravica

militaris aprili, majo.

apriti, majo.

aprili, majo.

aprili, majo.

aprili, majo.

\section{TYPHEAE}

Sparganium

Typha ramosuma

latifolia

minima junio, augusto.

majo, julio.

aprili, junio. 


\section{Sectio III.}

PLANTAE ACOTYLEDONEAE (1)

\section{EQUISETACEAE}

EQUISET⿱N⿴囗十

ramosissimum

fluviatile

\section{FILICES}

$\begin{array}{lll}\text { Adianthom } & \text { Capillus Veneris majo, junio. } \\ \text { Asplenom } & \text { ceterach } & \text { majo, junio. }\end{array}$

(1) Io mi sono astenuto di un rigore botanico nella indicazione delle Classi, Famiglie, Tribù ed altre suddivisioni nelle piante Crittogame, come pure nelle Fanerogame per tutte le Famiglie e Tribu rispettive; per la ragione che siffatto rigore scientifico mi avrebbe consumato molto tempo a frugare nelle opere botaniche. Essendo questo semplice cenno redatto a solo oggetto d'indicare le piante spontanee che vegetano nell' agro barulense, e non giả di presentare una flora, propriamente detta, mi sembra sufficiente la classificazione adottata, la quale per altro non si allontana dai cardini 


\section{FUNGI}

$\begin{array}{lll}\text { Polyporus } & \text { ovatus } & \text { septembri, octobri. } \\ \text { variegatus } & \text { septembri, octobri. }\end{array}$

\section{LICHENES}

LIGaEN

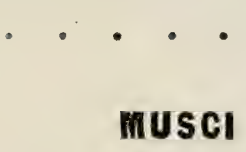

HYPNUM?

\section{TUBERACEAE}

LrCOPERdon pedunculatum septembri, octobri.

fondamentali della scienza Botanica. D'altronde, sendo io occupatissimo ai miei prediletti studii agrarii, ho ferma speranza che i Botanici vorranno onorarmi della loro indulgente equità, che pare, ne sia degno questo tenue lavoro. Inoltre è da avvertire, che dal 1847 fin oggi è facile come taluni nomi generici e specifici delle piante avranno avuto cambiamento secondo la nuova nomenclatura botanica ; quindi a rettificarli rigorosamente, mi conveniva riportarmi a consultare opere botaniche, la qual cosa non poteva fare perchè sono dedito a scrivere le LEZIONI DI AGRICOLTURA AD USO DE' PROPRIFTARî ISTITUITI. 


\section{ELEN C O}

DEI NOMI Volgari di TAlune PIANTE, I QUALI SI SONo POTUTI RICEVERE NERCÈ LE VARIE E REPLICATE RICERCHE FATTE PRESSO GLI ORTOLANI, I MIETITORI, I VIGNAJUOLI, I PASTORI, I MASSARI, E I CONTADINI D' OGNI GENERE.

NOMI VERNACOLI

Chiuppo

Atrigna

Crisciola lattarola

Occhio di pupa

Beccagallina

Erva di sepe

Jèta

Scinisco

Sailsaricddi, salsodde

Salsarieddi, salsodde

Salsarieddi, salsodde

Crispi

Fiore di ciuccio

Lattuchiello
NOMI BOTANICI

Populus alba

Prunus spinosa

Cynanchum acutum

Lychnis Githago

Stellaria media

Atriplex Halimus

Beta cicla

Chenopodium (tutte le specie)

Salicornia herbacea

Salicornia fruticosa

Salicornia macrostachya

Salsola soda

Calendula arvensis

Chondrilla juncea 
Scuppone o schiuppone

Spinedde

Sferruscina

Carduncieddi

Sevone

Crisciola

Rrecchie di preti

Erva di tetto

\section{Ruca}

Cavolo fore

Senapieddo

Cimamaredda

Rucascina

Mucchitiello

Cicorione

Scannacavallo

Rogna canina

Rocela viento

Tumo

Màmma dell' albero

Prufico

Juorno

Triemeto

Latierno

Accitieddo

Finucchieddo pe l'alive

Fergolazza

Sporchia

Squàcquera
Cnicus arvensis

Kentrophyllum lanatum

Picris spinulosa

Scolymus maculatus

Sonchus tenerrimus

Convolvulus arvensis

Cotyledon umbilicus

Sedum stellatum

Diplotaxis tenuifolia

Isatis tinctoria

Sinapis hispida

Sinapis incana

Sinapis alba

Daphne gnidium

Dipsacus sylvestris

Euphorbia falcata

Euphorbia ceratocarpa

Hypericum crispum

Thymus capitatus

Viscum album

Caprificus

Fraxinus excelsior

Olea europaea oleaster

Phillyraea media

Ammi majus

Foeniculum vulgare

Opopanax chironium

Orobanche pruinosa

Papaver Rhoeas 
Piseddo di scorzone

Lupino salvaggio

Lugurizia

Ciurufuogghio femminino

Ciurufuogghio masculino

Ciurufuogghio masculino

Ruveddascina

Schiatta pignatte

Scarapurcio

Perazzo, prazzo

Pricchiazzi

Sciosciole

Occhio di pupa

Ruca asinina

Cucumelle, cucumedde

Amaràscia

Vasa piedi

Sucamèle

Spine di sepe

Spine di Cristo

Pomidoro salvaggio

Stingio

Pistazzieddo

Canapazzo

Giglio di San Giacomo

Erva rossa

Erva a quadriello

Erva a quadriello

Scannacavallo
Anagyris foetida

Anagyris foetida

Glycyrrhiza glabra

Medicago scutellata

Medicago lappacea

Medicago histrix

Medicago falcata

Rumex conglomeratus

Mespilus monogyna

Pyrus cuneifolia

Portulaca oleracea

Zyzyphus vulgaris

Delphinium longipes

Reseda virescens

Rosa canina

Rubus dalmaticus

Tribulus terrestris

Hyosciamus albus

Lycium europaeum

Lycium europaeum

Solanum miniatum

Pistacia Lentiscus

Rhus coriaria

Vitex agnuscastus

Pancratium maritimum

Cyperus olivaris

Cyperus badius

Scirpus maritimus

Aegylops ovata 
Cannedda

Avenacchia

Grano di formica

Mustacci di militare

Coda di volpe

Coda di sorcio

Sciuglio

Cigliata

Cannazza

Coda di volpe

Avrusceno

Avuzzo

Lambascione

Cipolla canina

Fiore di lambascione

Fiore di monumento

Màmma dell’ acqua

Màmma dell' acqua

Màmma dell' acqua

Paglia gòglia

Paglia gòglia

Fungi di perazzo

Fungi di perazzo
Arundo donax

Avena sterilis

Hordeum murinum

Lagurus ovatus

Lagurus ovatus

Lagurus ovatus

Lolium maximum

Lolium multiflorum

Phragmites communis

Polypogon monspeliense

Asphodelus luteus

Asphodelus ramosus

Muscari comosum

Scilla maritima

Tulipa sylvestris

Tulipa apula

Lemna gibba

Lemna minor

Lemna trisulca

Typha latifolia

Typha minima

Polyporus ovatus

Polyporus variegatus

Questi sono tutti i nomi volgari che mi è riuscito di raccogliere in dieci anni di tempo, interrottamente però. Gli è certo intanto che molte altre piante hanno i loro nomi vernacoli nel linguaggio de'campagnuoli. Ove col \&empo mi si porgerà l'occasione di riunirne altri, lo 
farò con piacere; essendo mio pensiero che tutti $\mathrm{i}$ dettagli comuni pei nomi delle piante e per le località delle loro rispettive abitazioni, sono notizie preziose per ciascun paese; onde volendosi dai dotti Botanici distendere una flura perfettamente completa di qualche vasta Regione, non altrimenti si potrà raggiungere lo scopo se non che riunendo una quantità di lavori parziali e locali, che da per se facilitano la grandiosa opera nel loro gabinetto. Io ho fatto quel tanto che ho potuto, e spero tuttavia di continuare questo lavoretto in più ampie notizie, purchè avrò l'agevolazione di perlustrare di bel nuovo le patrie canpagne. Mi auguro d'altra parte che altri seguano il nio esempio: non sarà alcerto opera perduta, poichè si renderà servigio al paese e alla scienza. Che se la gioventù si persuaderà essere assai più utile lo studio delle scienze naturali anzichè quello de' perniciosi ed orrendi romanzi oltramontani, io sentirò in me la dolce gioja di aver data una piccola spinta ad una grande verila di economia sociale. L' individuo che diffondendosi nelle astratte speculazioni, non afferra mai un punto di utilità positiva per se e per gli altri; questo individuo, io dico, altro non è che una lenta cancrena la quale corrode l' animo ed il cuore a danno effellivo della sua morale e della sua ragione. L'immortale filosofo Ginmbatrista Vico in una sua lellera autografa, (fattami legrere dalla gentilezza dell'ottimo amico Artista sig. D. Filippo Trorsi) ed ancora inedita, scritta per apologia della sua opera, si doleva a ragione, quando diceva: Il GaSSENoI ritruovò il Mondo tutto marcio in amori di romanzi, e illanguidito in braccio di una troppo 
compiacente morale; e vivo udi da per tutto celebrarsi il suo nome di Ristoratore della buona Filosofia; perchè di un sistema, che fa criterio del vero il senso, di cui a ciascun piace il suo; e pone nel piacere del corpo, perchè non vi ’̇ altro per Epicuro, che vano, e corpo, l'umana felicità.

\section{SPECCHIETTI}

DEL NOMERo TOTALE DELI.E FAMIGLie, DE' GENER, DELLE SPECIE, E DELLE VARIETÀ.

\section{Famiglie}

DICOTILEDONI. MONOCOTILEDONI ACOTILEDONI .
66

5

\section{Generi}

DICOTILEDONI. MONOCOTILEDONI ACOTILEDONI .
268

69

6

\section{Specie}

DICOTILEDONI . 


\section{Varietà}

\begin{tabular}{|c|c|c|c|c|c|}
\hline DICOTILEDONI . & • & - & • & • & • \\
\hline MONOCOTILEDONI & & - & $e^{\circ}$ & $\bullet$ & \\
\hline ACOTILEDONI : & • & . & • & . & • \\
\hline
\end{tabular}

SOMME GENERALI

Somma delle famiglie . $\quad$. $\quad$. 83

Somma de'generi . . . . . 343

Somma delle specie . . . . . 758

Somma delle varietà . . . . . 43

\section{SPECCHIETTO}

DELLA PROPORZIONE MAGGIORE DELLE DICOTILEDONI

SULLE MONOCOTILEDONI.

Famiglie Generi Specie Varietà

$\begin{array}{lllll}\text { Dicotiledoni } & 66 & 268 & 605 & 37\end{array}$

$\begin{array}{lllll}\text { Monocotiledoni } & 12 & 69 & 145 & 6\end{array}$

Questo specchietto serve a dimostrare come si trova esatta la legge di Geografia bolanica esposta dal sig. DB Candolle nell' opera sua, volume secondo. Pour les régions temperées des deux hémispères, la proportion des Di- 
cotylédones argmente et celle des Monocotylédones diminue à mesure qu'on se rapproche des tropiques. Cette loi générale ressort de toutes les subdivisions du tableau, les pays étant disposés dans chacune selon leur latitude, en marchant $d u$ póle arctique au pôle antarctique. Toutefois, il y a de nombreuses modifications qui révèlent des influences secondaires. Ainsi, avec une température analogue, les pays humides offrent une proportion de Monocotylédones plus forte, et de Dicotylédones plus faibles; les pays secs, au contraire, présentent une proportion de Dicolylédones plus forte et de Monocotylédones plus faible. Questo è precisamente il caso della Puglia, ed il fatto lo dimostra. E se riscontriamo la Flora del Regno di Napoli del sig. cavalier Tenore, e la Flora Sicula del sig. cavalier Gussone, troviamo le stesse proporzioni maggiori nelle Dicotiledoni e minori nelle Monocọtiledoni. Di fatti nella Flora Napolitana sono registrate 2543 specie delle prime, e 589 delle seconde: e nella Flora Siciliana stanno rassegnate 2043 specie Dicotiledoni, e 507 Monocotiledoni. 


\section{NUNERO DE'GENERI PER CIASCUNA FAMIGLIA}

\section{Dicotiledoni}

\section{Famiglie}

Generi

Acanthaceae .

Amaranthaceae

Amygdaleae .

Apocineae

Asclepiadeae .

Capparideae .

Cistineae

Convolvulaceae

Cuscuteae

Hederaceae .

Hypericineae

Fumariaceae .

Jasmineae

Lineae

Lythrarieae .

Loranthaceae

Orobancheae. Oxalideae

Paronichieae. 
Plantagineae . . . . . . 1

Portulaceae . . . . . . 1

Resedaceae . . . . . . 1

Saxifrageae • . . . . 1

Smilaceae - . . . . . 1

Tamariscineae . $\quad$. $\quad$. $\quad$. 1

Valerianeae . . . . . . 1

Violarieae . . . . . . 1

Boragineae . . . . . . 2

Campanulaceae $\quad$ • $\quad$. $\quad$. 2

Caprifoliaceae . $\quad$. $\quad$. $2 \quad$. 2

Cucurbitaceae . . . . . . 2

Daphnoideae . • • • . $\quad$. 2

Gentianeae . : . . . . 2

Geraniaceae . . . . . 2

Moreae . . . . . . . 2

Onagraceae . . . . . . 2

Papaveraceae . . . . . . 2

Plumbagineae . • . $\quad$. $\quad$. 2

Pomaceae . . . . . . 2

Primulaceae - . $\quad$ • $\quad$ • 2

Santalaceae . . . . . . 2

Terebinthaceae $\quad . \quad$. $\quad$. $\quad$. 2

Urticaceae . . . . . . 2

Verbenaceae • • . • . . 2

Amentaceae . . . . . . 3

Dipsaceae . . . . . . 3

Oleaceae . . . . . . 3

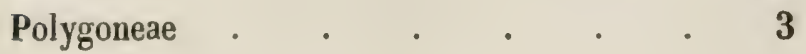


Rhamneae . . . . : . 3

Crassulaceae . . . . . . 4

Rosaceae . . . . . . 4

Rutaceae . . . . . . 4

Solanaceae . . . . . . 4

Chenopodieae . $\quad$. 5 . 5

Euphorbiaceae • • . . . 5

Malvaceae . . . . . . 5

Rubiaceae . . • . . . 5

Scrophularineae . . . . . 5

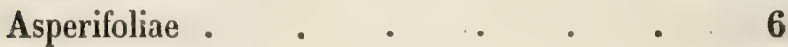

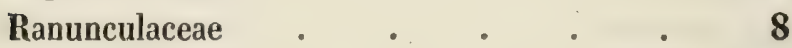

Caryophylleae • • • . . 12

Labiatae . . . . . . 13

Papilionaceae . . . . . 18

Umbelliferae . . . . . . 21

Cruciferae . . . . . . 23

Compositae . . . . . 50

Totale delle famiglie 66 Totale de'generi $\overline{268}$

Di tutte le famiglie Dicotiledoni abbiamo che il loro numero relativamente a quello dei generi decresce a misura che si aumenta la quantità di questi per una famiglia. Il seguente quadro chiarirà maggiormente l'idea mediante il numero delle famiglie di confronto al numero de'generi; messi tutti due i numeri in due colonne, quella a sinistra indicante le famiglie e quella a dritta mostrante i generi. 
QUADRO

Indicante la proporzione del numero delle famiglie di rincontro a quello de' generi.

Famiglic - delle quali ciascuna contiene - Generi

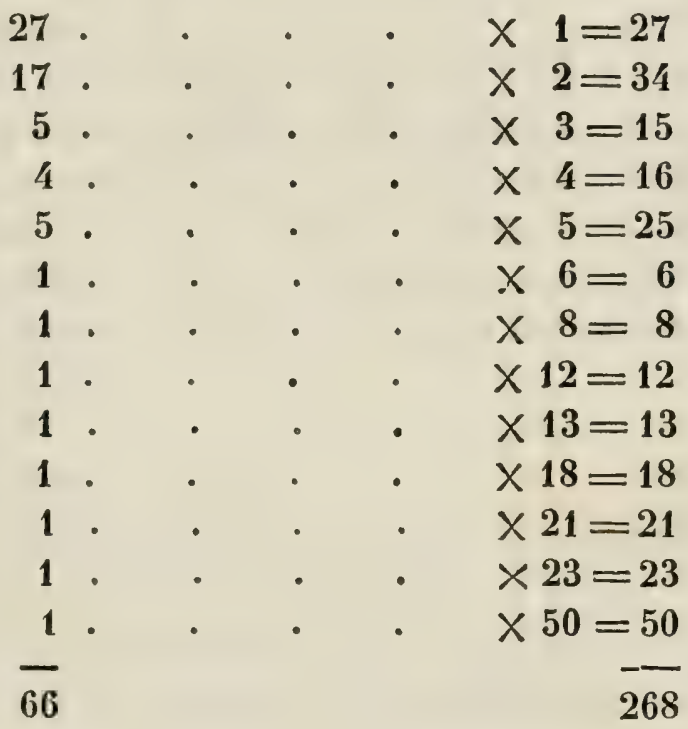

Ecco come chiaro emerge dall'antecedente quadro, che il numero delle famiglie, a un solo genere, è il più esteso: minore è quello delle famiglie a due generi, e cosi di se- 
guito, fino alla famiglia che contiene cinquanta generi. Di sessantasei famiglie Dicotiledoni, sei sono le più ricche in generi, cioè le Caryophylleae, le Labiatae, le Papilionaceae, le Umbelliferae, le Cruciferae, e le Compositae; delle quali quella delle Compositae è la più ricea, poichè ue contiene 50. E questo fatto naturale coincide esaltamente con quanto asserisce De Cindolle nel secondo volume della sua Geografia botanica, relativamente alla famiglia delle Compositae che è una delle più ricche in generi nella Flora generale della nostra Europa. Il Botanico Ginevrino riporta numerosi e belli esempii di questo genere; e dopo uno studio rigorosamente comparato, stabilisce le diverse leggi di Geografia botanica che sono un tesoro di vero sapere. $\mathrm{La}$ svariata e vasta erudizione della sua opera fa vedere quanto studio e quanta pazienza la sua nobile mente ha dovuto sostenere nel lungo giro di penose e brillanti vigilie, 
NUMERo DE' genERI PER CIASCUNA FAMIGLiA

\section{Monocoliledoni}

Famiglie

Generi

\begin{tabular}{lllllll} 
Alismaceae & $\cdot$ & $\cdot$ & $\cdot$ & $\cdot$ & $\cdot$ & 1 \\
Colchicaceae & $\cdot$ & $\cdot$ & $\cdot$ & $\cdot$ & $\cdot$ & 1 \\
Juncaceae & $\cdot$ & $\cdot$ & $\cdot$ & $\cdot$ & $\cdot$ & 1 \\
Amaryllideae & $\cdot$ & $\cdot$ & $\cdot$ & $\cdot$ & $\cdot$ & 2 \\
Aroideae & $\cdot$ & $\cdot$ & $\cdot$ & $\cdot$ & $\cdot$ & 2 \\
Typhaceae & $\cdot$ & $\cdot$ & $\cdot$ & $\cdot$ & $\cdot$ & 2 \\
Irideae & $\cdot$ & $\cdot$ & $\cdot$ & $\cdot$ & $\cdot$ & 3 \\
Najadeae & $\cdot$ & $\cdot$ & $\cdot$ & $\cdot$ & $\cdot$ & 3 \\
Orchideae & $\cdot$ & $\cdot$ & $\cdot$ & $\cdot$ & $\cdot$ & 3 \\
Cyperaceae & $\cdot$ & $\cdot$ & $\cdot$ & $\cdot$ & $\cdot$ & 4 \\
Liliaceae & $\cdot$ & $\cdot$ & $\cdot$ & $\cdot$ & $\cdot$ & 8 \\
Gramineae & $\cdot$ & $\cdot$ & $\cdot$ & $\cdot$ & $\cdot$ \\
\hline Tale delle famiglie 12 & \multicolumn{3}{c}{ Totale de' generi 69}
\end{tabular}

Nelle Monocotiledoni trovasi presso a poco la stessa decrescenza e proporzione, tranne qualche piccola differenza. Il seguente quadro esprimerà meglio il tutto. 
QUADRO

Indicante la proporsione del numero delle famiglie di rincontro a quello dégeneri.

Famiglie - delle quali ciascuna contiene - Generi

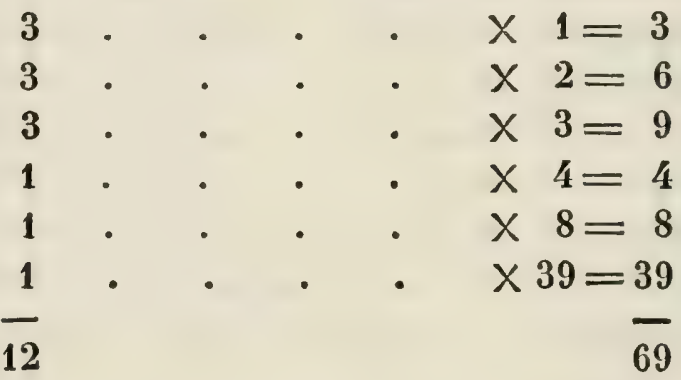

Di tutte le famiglie Monocotiledoni, tre sono le più ricche in generi; cioè le Cyperaceae, le Liliaceae, e le Gramineae, le quali ultime ne contengono 39 . Se mettesi a confronto la famiglia delle Dicotiledoni con quella delle Monocotiledoni, tutte due le più ricche in generi, si trova così :

Dicotiledoni : Compositae . . . 50 Monocotiledoni : Gramineae $\quad$ • $\quad 39$ 
Le prime dunque sono più ricche delle seconde nella proporzione di un quinto di più. E se facciasi il paragone tra le due famiglie importanti pel foraggio degli animali, si osserverà che le Graminaceae sono più ricche in generi che non lo sono le Papilionaceae; poichè le prime ne hanno 39, e le seconde solamente 18: cioè vi è una proporzione di più della metà. In quanto alle specie poi le $P a-$ pilionaceae superano le Graminaceae; giacchè nelle prime si numerano 86 specie e otto varietà, e nelle seconde si trovano $\mathbf{7 0}$ specie e cinque varietà.

NUMERo de' GeNeri PER CLASCUNA FAMigLia (1)

\section{Acotiledoni}

Famiglie

\begin{tabular}{lcccccc} 
Equisetaceae & $\cdot$ & $\cdot$ & $\cdot$ & $\cdot$ & $\cdot$ & 1 \\
Filices & $\cdot$ & $\cdot$ & $\cdot$ & $\cdot$ & $\cdot$ & 2 \\
Fungi & $\cdot$ & $\cdot$ & $\cdot$ & $\cdot$ & $\cdot$ & 1 \\
Musci & $\cdot$ & $\cdot$ & $\cdot$ & $\cdot$ & $\cdot$ & 1 \\
Tuberaceae & $\cdot$ & $\cdot$ & $\cdot$ & $\cdot$ & $\cdot$ & 1 \\
\hline
\end{tabular}

(1) Qui le famiglie sarebbero sei, giusta l' elenco della terza sezione a pagina 116 e 117; ma siccome mancano i generi de'LrcheNI, cosi ho calcolato per cinque. 


\section{QUADRO}

Indicante la proporzione del numero delle famiglie di rincontro a quello de' generi.

Famiglie - ciascuna contenente - Generi

\begin{tabular}{ccccccc}
1 & $\cdot$ & $\cdot$ & $\cdot$ & $\cdot$ & $\cdot$ & 1 \\
1 & $\cdot$ & $\cdot$ & $\cdot$ & $\cdot$ & $\cdot$ & 2 \\
1 & $\cdot$ & $\cdot$ & $\cdot$ & $\cdot$ & $\cdot$ & 1 \\
1 & $\cdot$ & $\cdot$ & $\cdot$ & $\cdot$ & $\cdot$ & 1 \\
5 & & & & & & $\frac{1}{6}$ \\
\hline
\end{tabular}

L'antecedente quadro delle famiglie e generi delle AcoTILEDONI, quantunque povero assai, e non da tanto da offrire materia da confronto, pure è stato da me disteso a solo oggetto di maggiore esattezza nella esposizione dei dettagli del presente lavoro.

In quanto ai quadri delle Dicotiledoni e MonocotileDONI cjascun comprende che la colonna numerica a sinistra presenta colla sola somma di tutte le cifre la quantità delle famiglie, come assai più avanti sta esposto nei diversi specchietti delle famiglie, generi, specie, e varietà: la colonna numerica poi a destra rappresenta la quantilà 
de'generi eguale a quella de' specchieuti, ma non colla somma delle cifre, ben vero mediante ciascuna cifra de' generi moltiplicata col numero delle famiglie corrispondenti. Così per esempio; se al primo quadro delle Dicotiledoni si moltiplica $2 \times 17=34$, e poi si faccia cosi per le altre cifre, si avrà la quantità de' generi. Mi si perdoni questo dettaglio, il quale ho esteso appositamente per dispensarvi o Lettore da siffatta fatica.

Avrei voluto, per massima esattezza, esporre in miglia quadrate la estensione del territorio barlettano, e riterire ad ogni miglio la proporzione delle specie, seguendo cosi l'esempio di De Candolle, di Gussone, e di altri Botanici, ma sventuratamente siffalte notizie mi mancano. Ad ogni modo riporto per ora quel poco che ho potuto racgliere dalla gentilezza del sig. D. Giuseppe Italia, che le ha rilevate dal catasto fondiario. Tutto il tenimento barulense sarebbe di 11735 versure dell' antica misura pugliese, le quali, ridotte a moggia legali, darebbero una cifra elevatissima. Sono ripartiti siffatti terreni in prima, seconda, e terza classe, conforme ai varii gradi di loro fertilità e valore. Circa una tanta estensione fo osservare, che avuto riguardo alla spontanea vegetazione, io intendo solamente includere i raggi che dal lido del mare, sotto la Città, si distendono così, cioè :

$1^{0}$ Raggio verso l'Ovest e il Sud-Ovest, passala la fiumara ed il fiume, sino al di quà della strada nuova che conduce al novello villaggio $S$. Ferdinando (una volta $S$. Cassano), e precisamente fino al punto ove le acque dell'Ofanto traversando la via, vanno a scaricarsi in buona parte nel lago di Salpi. 
$2{ }^{\circ}$ Raggio al Sud-Sud-Ovest e al Sud sino alla fontana di Canne, e sulla stessa linea fino alla mezza via che conduce a Canosa.

$3^{\circ}$ Raggio al Sud-Sud-Est e al Sud-Est verso la metà della via di Andria e un miglio al di là della contrada di Santo Luca.

$4^{0}$ Raggio ed ultimo all' Est fino alla metà, della strada di Trani, e nella contrada delle paludi fino al punto ov' è la famosa vasca di acqua piuttosto dolce, la quale vasca vien denominata bocca d'oro. Non bisogna confondere i raggi della circonferenza che naturalmente offrono le campagne del paese colla possessione propriamente detta. Cosi v'ha de' proprietarii barulensi che hanno fondi presso altri Comuni, come del pari vi sono possidenti di Andria che hanno beni presso l'Ofanto ec. Ho voluto ciò avvertire, per la ragione che essendo tutto botanico l'oggetto del presente lavoro, non si abbia la debolezza di affacciare quistioni ed osservazioni ingiuste da quei pochi individui che non sono avvezzi a gustare gli studii scientifici.

Mi si permetta intanto far notare che alla pagina 125, quando espongo la cifra delle Dicotiledoni maggiore a quella delle Monocotiledoni per le due Flore di Tenore e GusSONE, non ho voluto rigorosamente riguardare la esatta quantità delle cifre, benvero sostenere con una pruova di più la legge della distribuzione geografica delle piante sulla superficie terrestre. 


\section{NOTE ED OSSERVAZIONI}

ACantnus spinosus. Vegeta ai margini della strada consolare che conduce a Trani, tre in quattro miglia distante da Barletta; e precisamente in quel sito elevato dopo l' avvallamento, di cui parlai nell' esposizione della posizione topografica delle campagne barulensi, poco lungi dal tratturo.

Populus nigra. Il pioppo nero, per quanto è a mia conoscenza, non è spontaneo. I suoi rami, che dalle campagne della Provincia di Avellino sono trasportati dalle acque del fiume, facilmente mettono spontaneamente radici se per l'impeto delle correnti restano sotterrati in parte, e le nuove messe si sviluppano ed allungano da maggio in poi. Io ne osservai e raccolsi diversi nella state del 1847 presso la fiumara, e son sicuro che diventerebbero alberi belli, se i possidenti ne prendessero cura. Presso l' Ofanto vi sono ricchissime masserie, tra le quali una ve n'ha denominata il chiuppo, giacchè gli antipassati padroni vi fecero una piantagione di pioppo nero che riusci a meraviglia. In quel medesimo anuo ce n' erano pochi avanzi, e tutto il resto era distrutto. I signori De Martino ne avevano fatta una novella piantagione lungo il canale che dal- 
l'Ofanto conduce le acque nel lago di Salpi per tutto l'anno. Le piante prosperarono assai, ma non tutte furono rispettate dalla violenza delle correnti. Quei novelli piantoni furono fatti venire da Terra di Lavoro. Speriamo che gli altri proprietarii ne seguano l'esempio, ed impieghino tutte le cure onde quei luoghi silvestri siano coperti da alberi utili e non dalla così detta rogna canina (Euphorbia ceratocarpa) che è la vera vergogna e barbarie di quei luoghi cotanto fertili.

Salix Villarsiana. Questa bella specie di Salice presenta un certo che di ornamento e di romantico vicino le acque del fiume, ove la sua dritta e pomposa chioma fronzuta si eleva assai infra $\mathbf{i}$ cespugli ed $\mathbf{i}$ macchieti. In agosto, allorchè i suoi frutticini si schiudono e metton fuori loro semi forniti di morbidissimo e serico tomento bianco, l'albero acquista un colore argentino che in mezzo a denso fogliame, percosso dai raggi solari, risalta agli occhi, più o meno brillante, ad ogni agitar di vento.

Salix purpurea. Le punture degl'insetti producono escrescenze carnose sulle foglie di questo Salice, le quali escrescenze sono sferiche e di un rosso vivo lucido con macchia nera al di sotto della puntura, che a prima vista e senza prevenzione somigliano ai semi della Glycine precatoria.

Prunus spinosa. Si deve questa specie considerare come veramente indigena, o come avanzo di antica coltivazione?

Cynancum acutum. Il volgo esprime bene la proprietà assai lattiginosa di questa pianta colle parole crisciola lat- 
tarola. Il suo fogliame particolare accresce bellezza a' suoi fusti lunghi e volubili nei terreni feraci.

Heliotropium stpinum. Questa specie nasce nei terreni pantanosi vicino le foci dell' Ofanto.

Capparis sicula. Le tenere cime di questo suffrutice si raccolgono in aprile e in maggio, e si fanno raddolcire nell' acqua ogni $\mathbf{2 4}$ ore per sei o dieci giorni: indi si conservano nell'aceto, e se ne fa uso come insalata. In quanto all' uso de'suoi fiori non aperti ( comunemente chiapperini) è cosa conosciuta da tutto il mondo. Nel 1837 ne vidi una estesa industria a Manfredonia nella Provincia di Foggia. La città di Trieste ne faceva grande acquisto: nıa una veduta di economia pubblica per parte del Governo Austriaco ne diminuì lo smercio. Questa specie di Capparis è spinosa, e reca grave molestia a'contadini che ne raccolgono i fiori non per anco schiusi. Sarebbe a desiderare la introduzione della Capparis rupestris la quale non porta spine ed è spontanea a Posilipo presso Napoli e sulle rupi che sono alle spalıe del Convento di Piedigrotta. La moltiplicazione sarebbe facile, poichè, raccolti i semi ben maturi nell'Autunno, si conservano in sito asciutto durante il verno, in aprile si mescolano col cemento da fabbricare, a guisa di una pasta, e cosi disposti si affidano nei crepacci delle parieti ove abbondi l' elemento calcareo. A questo modo pruovano assai bene. Io ne ho vista sopra un muro una bella piantagione cosi procurata, ed assai florida, a segno da fare invidia alla stessa sua spontaneità. La osservai con somma soddisfazione a Posilipo in ottobre $\mathbf{1 8 5 6}$, ove ne raccolsi parec- 
chi saggi, parte fioriti, e parte fruttificati, in compagnia del sig. D. Vitantonio Cappelli, giovine di grandi speranze nello studio dell' Agricoltura.

Gypsopityla Arrostir. Questa pianta fu da me raccolta per la prima volta in agosto 1844 nelle campagne di Canosa, e nel 1847 poi la rinvenni in quelle di Barletta e precisamente nella masseria del sig. D. Ferdinando Cafiero. La sua grossa e lunga radice è molto sproporzionata alla ramificazione piuttosto esile anzichè no. I contadini mi dicevano che essi adoperano la radice di questa pianta per lavare i loro panni di lana, poichè fa le veci del sapone quando viene strofinata nell'acqua. Saranno forse i suoi principii chimici identici in natura ed in proporzione a quelli della Saponaria? Senza dubbio sono due piante della stessa famiglia naturale delle Caryophylleae. Sarebbe a desiderarsi che i Professori di Chimica organica se ne occupassero. Non me ne sovviene il nome volgare.

Lycnis GithaGo. I fanciulli nel fare fantocci di carta o di pannilini, sogliono servirsi de' semi neri di questa specie di pianta nel situarne a posticcio gli occhi: e siccome il fantoccio volgarmente lo chiamano pupa, così riconoscono i semi e la pianta col nome di occhio di pupa o di pupillo.

Saponaria vaccaria. Abita dirimpetto al mare vicino al castello, e propriamente di vis-à-vis alla così detta mùmma d'Aré (Madre di Eraclio), lungo il margine de'cam$\mathrm{pi}$, in terreno esposto a bacio, marnoso ed erboso. La $S a$ ponaria officinalis poi cresce presso l'Ofanto, e sembra che le piante provengano da semi trasportati dalla Provincia di 
Avellino mediante le correnti del fiume. La Saponaria officinalis l'ho raccolta a Monteforte, a Grottaminarda, ad Ariano, al valle di Bovino, e a Cardito di Caivano. Piacerà, credo, quì riportare poche parole storiche sulle voci popolari màmma d'Aré. Gli è un pezzo di rozza pietra calcare impiantato nei terreni silicei arenosi. Nel 1829 io me la ricordo bagnata dalle acque del mare per due palmi di profondità: e nel 1847 la osservai a secco, essendosi le acque marine ritirate per buon tratto, come più avanti dissi. Si vuole per tradizione storica che in quei contorni naufragò, un tempo, la nave entro la quale era il busto in bronzo fuso dell' attuale statua di Eraclio che regge nella piazza di Barletta. Il volgo pronunzia barbaramente Aré invece di Eraclio; e dalle sue parole sembra che quella spiaggia sia da esso considerata qual genitrice di Eraclio, che prima annegò nell' arena, e poi rinacque a novella vita mediante l'arte dello statuario greco Fabio Albano che gli formò le gambe e le mani. Ma su di questo argomento, o Lettore, consultate la storia, la quale, se mal non mi ricordo, riferisce che si suscitò quistione tra i Barlettani e i Tranesi di allora, se dovevano i primi o i secondi appropriarsi di quel busto di bronzo fuso ch'era diretto per Venezia, e che sventuratamente naufragò in quei paraggi.

Silene bipartita. Il sig. Gussone nella sua Flora dell'isola d'Ischia, dopo di avere passate a rassegna tutte le varietà di questa specie, si esprime cosi: Omnes enumeratae varietates, quae in Sicilia proveniunt, excepta varietate A, quae in Apulia prope Barletta invenit Bruni, diffe- 
runt a Silene vespertina RETz. pubescentia adpressa ac tenui etc. etc. Gussone : Enumeratio plantarum vascularium in insula Inarime. Neapoli, 1854.

Silene rubelda. Nella stessa Flora d'Ischia trovasi cosi detto: Innumera specimina $S$. rubellae, quae in Apulia in vineis prope Barletta collegit Bruni, cum illis aegyptiacis a Cl. Kralichio missis, et cum icone DeliL. descr. de l'Egypt.t. 29. f. 3. ex asse quadrant, nec unquam in S. turbinatam transeunt; atque haec ab illa prima facie dignoscitur etc. etc. Queste notizie sulla Silene bipartita e sulla Silene rubella dimostrano come il sig. Gussone conserva nel suo famoso erbario i saggi delle varie piante in diverse epoche da me raccolte nell'agro barulense. $\mathrm{E}$ si rileva inoltre un leggierissimo sentore a favore della mia opinione relativamente al raggio esteso, fin sulle coste dell'Adriatico, della naturale e geografica abitazione della Scilla undulata, siccome in appresso dirò. La Silene rubella nasce spontanea nei vigneti della contrada detta del crocefisso. Ivi fa pompa de' suoi gentili fiori color di rosa dopochè le viti sono state sarchiate e rincalzate in aprile ed in maggio. Plante cultivée malgrè la volonté de l'honme.

Silene conica. Vegeta nei terreni silicei presso l' Ofanto.

Silene muscipula. Coabita colla Saponaria vaccaria.

Atriplex Halinus. Questo frutice si coltiva per siepi, o isolato o in compagnia del Lycium europaeum. Il suo portamento acquista colossali dimensioni tanto pel terreno che pel clima. Forma bellissime difese agli orti, particolarmente contro i venti impetuosi, mercè le sue nume- 
rose e intrecciate ramificazioni. Il cavallo, l'asino, la pecora, e la capra lo mangiano avidamente. Ignoro però se sia del pari appetito dagli animali vaccini. L'Atriplex $\mathbf{H a -}$ limus, come altra volta dissi, si diletta dell' aria marina. Del pari il suo compagno, il Lycium europaeum, che non solo ho raccolto sulle coste dell'Adriatico, ma ben anche su quelle del Tirreno lungo il golfo di Napoli, e precisamente presso le due Torri, a Resina, a Portici, ed a Pozzuoli. Il basso popolo a Barletta raccoglie le tenere cime del Licio in Primavera, le allessa, e poi le condisce e mangia a mo d'insalata.

BETA CiCLA. I campagnuoli e la gente povera raccolgone quest'erba, la Sinapis hispida, la Sinapis pubescens, la Brassica fruticulosa, la Diplotaxis tenuifolia, il Sonchus tenerrimus, il Sonchus oleraceus, il Cichorium intybus, qualche altra pianta, e ne fanno il loro prediletto camangiare denominato volgarmente fogghie mischiate.

Chenoponium virine. Tutti sanno che le specie di Chenopodium, tranne poche eccezioni, tramandano, chi più chi meno, un fetore insopportabile. Vuolsi da taluni, che queste piante fetenti, introdotte nel vino, lo alterano e quindi lo corrompono del tutto. Io non ne ho fatte mai esperienze. Prosperano in vegetazione in tutli gli orti e negli altri luoghi ove sono macerie grasse e ove si depongono letami ed altre sostanze fertilizzanti.

Salicornia nerbacea. Tutte le specie di questo genere addimandansi colle voci comuni salsodde e salsarieddi; e pare che queste parole de' campagnuoli e pescatori cor- 
rispondano bene al vero significato di piante che crescono in siti pantanosi e salini.

Salsola soda. Vogliono taluni che il basso popolo ne mangi le tenere cime, sia in insalata, sia cotte e condite con olio. Io ricordo nella mia prima gioventù contadini che spesso trasportavano in Città panieri pieni di questa pianta, ma ne ignoro positivamente. a quale uso la destinavano.

Artemisia coerdlescens. Abita alle paludi, e precisamente nelle vicinanze del lago così detto di aresciàmn. (Pronunziate l' $e$ muta). Poche volte ne raccolsi i saggi tra la state e l'autunno: ma molti altri in più fiate io mi riceveva in Napoli mediante la bontà de' fratelli Russo che a mie istanze mi favorivano premurosamente inviare.

Chondrilla Junces. Ce ne sono tre varietà, delle quali una porta le foglie runcinate alla base e lineari lungo il fusto: la seconda è fornita di foglie lineari e glabre dal nodo vitale sino alla cima: la terza in fine le ha quasi lanceolate ed ispide o spinulose. Abbondano nei vigneti, come altra volta feci avvertire, parlando de'terreni; ed i vignajuoli ne mangiano le tenere cime prima di fiorire. Questa specie è una di quelle, che in termini generali geografico-botanici il signor De Cannolle chiama plantes cultivées malgrè la volonté de l'homme.

Cimpsantuemum coronariom. Vi è la varietà a fiori di color giallo carico, e l'altra a color giallo biancastro. Ai margini de' campi e nei fondi arenosi silicei presso mare abbondano a preferenza. 
Cichoriom intybus. Ve ne sono due varietà: quella ciod a fior celeste e l'altra a fiore color di rosa. Il pregio della cicoria selvatica è conosciuto da tutto il mondo: ma chi non mangia le cosi dette cimccicorie in aprile e in maggio, non può saper apprezzare la vera amenità campestre delle praterie pugliesi, nè saper riconoscere il beneficio che la propria salute ne risente.

Cricus arvensis. Gli animali cavallini e pecorini, particolarmente i primi, ne mangiano avidamente le foglie allo stato di tenerezza, momento in cui le piccolissime spine, di che vanno fornite, sono innocue al loro palato. Gli è questa una pianta che infesta orribilmente i campi ed i vigneti. Nel tenimento di Caivano e di Cardito anche l'ho raccolta, ma è rara, perchè ivi scarseggia l'elemento calcare-argilloso di cui si diletta.

Contza ambigda. Questa specie fu da me rinvenuta solamente nel giardino del palazzo di Fraggiani e nel chiostro del Convento di Santo Andrea. Tanto il palazzo quanto il Convento sono vicini al mare; il primo alla parte settentrionale della Città, ed il secondo verso il Nord-Est della medesima. La specie in parola non fu trovata da me in nessun altro sito, e neppure nel chiostro del Convento di San Giovanni di Dio, e nell'atrio del quartiere del Carmine, e nel chiostro del Castello; i quali siti trovandosi sulla stessa linea settentrionale della Città, mi destarono sospetto che ivi avessi potuto trovar la pianta, ma inutili furono le mie ricerche più volte ripetute. In luoghi adjacenti non mai l'ho vista, e nelle campagne molto meno. Il dottor CASALE mi assicurava la stessa cosa. Ora, mercè 
questi fatti, chi potrebbe rintracciare l'origine della esistenza della specie nei due luoghi sopra citati prima della fondazione delle loro fabbriche? Sarebbe stata forse la pianta introdotta negli scorsi anni mercè il traffico della gente commerciante?

Hypochoeris Tenonn. Vegeta nei terreni argillosi e marnosi a bacio dirimpelto il mare, ed ivi acquista portamento colossale di cinque a sei palmi. I suoi fiori gialli, esposti all'ombra, risaltano assai sul verde cupo del lungo ed ispido fogliame.

Kentrophylum lanatum. La voce volgare spinedde de' vignajuoli (di cui si servono per indicare la pianta) concorda molto al fatto, poichè nel tempo della piena fioritura le spine della pianta, e particolarmente quelle che stanno intorno al capolino, sono assai rigide. E se si aggiunge la natura calcarea del terreno, il clima secco, e la stagione calda di luglio ed agosto, si riconoscerà di leggieri come queste circostanze fisiche favoriscono a consolidare la rigidezza delle spine acute e pungenti.

Picris spinulosa. Abita nei terreni sottomarnosi, e nei vigneti. I vignajuoli l'addimandano sferruscena, che nel linguaggio popolare vuol dire piccola lima che stropicciando sul ferro, arreca alle orecchie quella sensazione di suono aspro e dispiacevole. In verità la pianta è quasi tutta coperta di pelame piuttosto rigido; cosicchè al tatto se ne avverte la ruvidezza. Se leggiamo la descrizione fitognostica de'Botanici, troviamo molto del vero, ed uniforme all' idea de' contadini. Ecco quanto ne dice il sig. GussoNE nel secondo volume della sua Flora Sicula. - Her- 


\section{$-147-$}

bae biennes, aut perennes, plus minus pilis furcatis hirsutae: folia indivisa, vel sinuato-dentata: flores lutei, subcorymbosi. - Pilis furcatis patentibus setisque hirta, caule erecto angulato-sulcato ramoso, foliis oblongo-lanceolatis dentatosinuatis, superioribus amplexicaulibus integris, floribus subcorymbosis, pedunculis non incrassatis, anthodii villosi foliolis ad carinam incrassato-spinulosis, exterioribus semipatentibus apice subsquarrosis, pappo sessili. - Caules solitarii, in umbrosis fere orgyales, superne alterne ramosi; ramis erectis; folia tuberculato-hispida: etc. etc. E più appresso lo stesso Botanico dice: Differt a $\boldsymbol{P}$. hieracioide hirsutie majore, anthodii foliolis charactere: etc. etc. In altra parte riportando i sinonimi della specie, secondo il PANphiton di Cupani, riferisce cosi: Hieracium asperum Hieracium hirsutum: etc. Tutti questi caralteri si accordano col significato della voce volgare sferruscena. (Pronunziate l'e muta).

Scolvuus maculatus. Porta le foglie radicali con macchie bianche, e nei giorni festivi di Pasqua si mangia sotto il nome di carduncieddi salvaggi.

Scorzonera Colunnae. La raccolsi in maggio del 1847 nelle praterie naturali sopra gli ameni colli della famosa Canne, e propriamente nelle vicinanze dell' Ofanto, ov'è la cosi detta masseria del medico.

Convolvulus arvensis. I campagnuoli la distinguono colla voce crisciola. Per quanto mi ricordo, questa parola presso i contadini significa una specie di nastro o di laccio da servire per ligare o affasciare una cosa qualunque: ed in vero spesso i medesimi si servono de'fusti lunghi e ser- 
peggianti del Convolvulus arvensis per ligare piccoli fasci di erbe, pampani, stoppia, o altro verdume.

Cotyledon umbilicus. Non vi è dubbio che non tulti i nomi vernacoli delle piante hanno rigorosamente una stretta coincidenza di significato colle specie alle quali si riferiscono: ma è certo che generalmente parlando serbano sempre un' origine allusiva ai fatti naturali delle piante stesse. Quest' erba grassa viene addimandata orecchie di preti: ora in questo caso che relazione d'idee vi è tra le foglie del Cotyledon umbilicus e le orecchie de'Preti che sono orecchie come quelle di tutti gli altri? Secondo me si è nella tradizione delle antiche usanze che bisognerebbe rinvenire l' origine di queste voci volgari. Sarebbe uno studio di curiosità e di pura e semplice crudizione storica del paese. Nella Geografia botanica del sig. De Candolle spesso si parla di siffatte ricerche per facilitare lo studio sull'antichità delle varie piante e specialmente sulla patria vera di quelle coltivate nelle diverse parti del globo.

Crassela Magnolit. Cresce nei siti petrosi e sassosi delle praterie naturali che sono tra Barletta ed Andria. Mio Fratello me ne mandò i saggi a Napoli.

Sedum stellatum. Abita su pei tetti, ove fa pompa de'suoi numerosi e vaghi fioretti color di rosa.

Biscutella apula. Vegeta nei pascoli aprici sul cosi detto tratturo.

Brassica erdGa. Nelle praterie dell'Ofanto se ne trova a dovizie con fusti alti tre palmi circa. E una buona pianta per soverscio, come si pratica in Terra di Lavoro.

Diplotaxis tenuifolia. I villani delle masserie allessa- 
no quest' erba allo stato di tenerume insieme col pane di campagna, e poi la condiscono con olio e sale, mangiandola avidamente. Siffatto cibo rustico vien riconosciuto coi nomi di pan colto e ruca, e forma l'ordinario camangiare di quella povera gente. Gli è certo però che a molti piace; ed io credo che non influisce in ciò il solo forte appetito prodotto dall'aria campestre, bensì l'abbondanza del glutine in quella qualità di pane bruno che dicesi perrozzo o perruozzo.

Isatis tinctoria. Questa pianta tintoria prospera nei vigneti a terreno calcare soltomarnoso, si eleva sino a quattro palmi di altezza, e porta copioso e largo fogliame glabro. I vignajuoli l' addimandano cavolofiore; ed in vero la sua infiorescenza somiglia un tantino a quella del cavol fiore comune che si coltiva negli orti, comecchè tutte due queste piante appartengano alla stessa famiglia naturale delle Cruciferae.

Nasturtium officinale. Abita alle paludi; e nel maggio 1847 ne raccolsi un saggio presso le acque della fontana di Canne poco lungi dall' Ofanto.

Sinapis incana. Nella primavera i fusti ancor teneri di questa pianta vengono raccolti e venduti pel paese sotto il nome vernacolo cimamaredde. Questa parola esprime chiaramente l' idea di un sapore amaretto: ed è così. Gli abitanti ne mangiano con piacere, che più si accresce con quell' amaretto, che lusinga anzi che no. Durante la quaresima siffatto verdume forma il prediletto cibo delle famiglie.

Cuscuta minor. Le Cuscute apportano qualche danno 
positivo ai pascoli delle pecore là ove spesso le ho raccolte. Attaccano indistintamente tutte le piante leguminose e graminacee di quei prati. Soventi volte le ho raccolte sul Thymus capitatus. I possidenti con venti o trenta carlini l'anno di spesato potrebbero fare aperta guerra alle $\mathrm{Cu}$ scute che danneggiano i loro pascoli; c dopo tre o quattro anni di pazienza ne sarebbero pagati ad usura con vantaggio effettivo delle loro pecore. Un colpo di zappa, un poco di calce caustica, un poco di fuoco, ed ecco tutta la cura e lo spesato.

Daphne GMidiem. Questo frutice infesta orribilmente gli erbaggi dell'Ofanto : se i proprietarii lo estirpassero un poco la volta, essi guadagnerebbero nei loro pascoli il venti per cento. Basterebbero quaranta in cinquanta ducati di spese in due anni per una così detta mezzana di terreno, ed il vantaggio sarebbe assicurato. Cosa sono $\mathbf{5 0}$ ducati di spesa in due anni per un possidente che sta alla testa di 60000 ducati? \& questa una pianta medicinale.

Scabiosa grandiflora varietas prolifera. Cresce lungo la strada, alle spalle del giardino del Convento de' cappuccini.

Scabiosa ... ? Nei terreni marnosi a bacio dirimpetto il mare, vicino le fornaci per la fabbrica de'mattoni e presso il casino del fu Marchese Affaitati nasce un' altra specie di Scabiosa diversa dalle altre, i di cui saggi confusi fra tanti altri di piante diverse, non potei rinvenirli nel mio erbario agronomico, e quindi non ho potuto sottoporli al giudizio del signor Gussune per assicurarmi precisamente a quale specie si appartenessero. 
Euphorbia falcata. Questa pianta vegeta nelle stoppie dopo la mietitura, ove gli animali vanno a pascolare. E probabile il supporre che i cavalli mangiandone, ne abbiano a sentir danno. Senza ulteriori ricerche e indizii sul fatto, non si potrebbe diversamente sospettare sul perchè, essa sia riconosciuta col nome di scannacavallo. A meno che non roglia dirsi che la causticita del suo succo lattiginoso sia sommamente nocivo agli animali nella stagione estiva.

Etphorbia ceratocarpa. Queslo perniciosissimo vegetabile per la feracità dei terreni raggiunge l'altezza di otto palmi e più, misurata spesse volte da me nel mese di maggio. E tale e tanta la sua abbondanza, che copre la terza parte di tutte quelle praterie, lungo le rive del fiume, ove cresce. Supponete una mezzana di venti versure, siate certo che circa selte versure per causa di questa pianta non dànno alcun prodotto. Così il terreno è perduto infruttuosamente, ed il vegetabile in parola forma la vergogna ed il barbarismo di quei luoghi! I possidenti spesso si dolgono che i loro erbaggi poco o niente fruttano! Ma che debbono fruttare, se la pianta assorbe la terza parte de'prati, tanto per le radici che per lo spazio che occupano i numerosi fusti e rami?

Vitis vinifera sylvestris. Ce ne sono due varietà vicino l'Ofanto; una a foglie dentate con peli aracnoidei, e l'altra con foglie profondamente incise con peli cellulari. Io ho raccolto quella a frutto bianco e quella a frutto nero. Non mi ricordo bene se la varietà bianca corrisponde a quella con foglie dentate, o alla seconda con foglie as- 
sai incise. Parecchi anni or sono ne presentai i corrispondenti saggi al Reale Istituto d'Incoraggiamento in virtù della dotta Memoria scritta e pubblicata dal sig. cavalier Tenore. Gli acini maturi li ho visti sempre della grossezza maggiore di quella del pepe. Il loro sapore è acidulozuccherino con vinacciuoli non molto grossi. Le piante crescono rigogliose a segno tale, da fare grandi macchieti con lunghissimo tronco. Stando ai principii della Geografia botanica del celebre De Candolle, la vite selvaggia vicino l'Ofanto è aborigèna di quei luoghi, o è avanzo di antiche coltivazioni? Io opinerei riservatamente e modestamente per l'esistenza aborigèna, poichè i siti, ove cresce la vite selvaggia lungo il fiume, hanno tutta l' apparenza del suo antico letto, e che il detto fiume divergendo le proprie acque dal lato opposto, doveva necessariamente lasciare a secco terreni feracissimi, i quali poi si sono coperti di svariata e superba vegetazione, di cui fa parte la vite silvestre. Se non che si potrebbe dire che nei passati secoli i colli che sono a cavaliere dell' Ofanto fossero stati coperti di vigneti, e che in tal modo la vite siasi diffusa in quei silvestri luoghi, sia per le antiche ceppaja e radici, sia pei semi. Che che ne sia, gli è questo un argomento che solo si potrebbe illustrare e chiarire, frugando nella storia degli antichi Romani, ai tempi di Canne, e degli altri Principi e Guerrieri che dominarono in quelle Provincie. Io ho voluto con riservatezza manifestare la mia opinione e non più. Pertanto mi gode l'animo riportare il passo del sig. De Candolce sulla vite, che tanto saggiamente espone nella sua opera, quando parla 
della origine geografica delle specie di piante coltivate. Eccolo. - "Vitis vinifera $\mathbf{L}$. La vigne est spontanée dans " toute la région inferieure du Caucase, au nord, et sur" tout au midi de la chaine, en Arménie, et au midi de " la mer Caspienne, d'après plusieurs auteurs cités par "Ledebour (Fl. Ross., I, p. 458). Les notions histori" ques ne permettent pas de douter que ce ne soit la pa" trie originaire de l'espèce. S'étendait-elle beaucoup vers " le midi de la Russie, l'Anatolie, la Perse, et les monta" gnes du Caboul? C'est ce que les recherches botaniques " ne peuvent plus nous apprendre. La Vigne se sème et " se naturalise aisément dans les régions tempérées de " l'ancien monde. Quand on trouve des pieds sauvages " daus les haies, les broussailles, rien ne peut apprendre a s'ils descendent de raisins cultivés ou de raisins sponta" nés, et plus on se rapproche de l'habitation originaire, " plus l'incertitude augmente. La conformation des feuil" les, la grosseur et la faveur des fruits sont des indices " trompeurs à cet égard, puisque des Vignes livrées à el"les-mèmes, non taillées, venues de semis au lieu de " boutures, peuvent très-bien offrir des caractères di" stincts, tout en provenant de Vignes cultivées. Il est " cartain que l'espèce prospère dans toute l'Asie occiden" tale tempérée, notamment au Caboul, en Cachemir et " dans le pays de Kunawur (Royle, Ill. Him., p. 148). "Elle a un nom sanscrit, Draksha, qui montre l'encien" neté de son existence ou de sa culture dans l'Inde se" ptentrionale. Monsieur Bunge l'a vue dans le nord de la "Chine, cultivée seulcuent (Enum., p. 11). Le progrès 
" de la culture dans l'Europe occidentale, par l'influence " des Grecs et des Romains, est résumé dans l'excellent " ouvrage de Reynier, sur l'Économie publique, et rurale " des Celtes et des Germains (I vol. in $-8^{\circ}$, Genève, 1818, " $p .472$ ). " Ecco dunque ciò che dice il Botanico filosofo ginevrino sull' origine e su i luoghi natali della vite nel secondo volume della sua opera; e pare che la mia opinione vada un tantino di accordo colla sua. Ed io mi reputo sommamente fortunato quando incontro che il mio pensare in talune cose scientifiche non è dissimile da quello de'Dotti. Lo stesso Autore in una nota a parte sull' abilazione della vigna, pronunzia queste parole. " La vigne " et le raisin ont reçu des noms extrèmement différents " dans les langues d'Asie el d'Europe, ce qui fait suppo" ser une habitation primitive fort étendue."

Hypericun GRISPUM. I vignajuoli l'addimandano rocela viento: ora queste parole vernacole sono molto espressive, dal perchè alludono ad un fatto tutto naturale e semplice. La pianta dell' Hypericum crispum porta fusti ramosi e disposti in croce, $\mathrm{i}$ quali sono talmente intrecciali fra loro, che l'intera pianta sino a un certo punto assume la furma sferica, tutta carica di fiori gialli. Dopo la fruttificazione seccano i rami dalla base in sopra, e senza disgiungersi fra loro, si distaccano dalla radice perenne, e mercè la loro leggerezza restano in balia del vento, il quale lungo i campi li spinge innanzi rotolandoli a mo' di palla; d'onde il nome di rocela-viento, che starebbe benanco applicato alla Phlomis herbaventi. L'Hypericum crispum è pianta nociva alle pecore, e cresce frequentemente nei vigueti mal 
coltivati e nelle stoppie dopo la raccolta de'cereali e di altre granaglie.

Marrubium Alysson. Abita nei pascoli aprici del tratturo e degli ameni colli di Canne.

Salvi argentea. Vegeta sulle aride piagge calcari verso Andria e verso Canosa.

Tuyuus Acrnos. Nasce sulle tufare e luoghi simili. Io l'ho raccolto copiosamente vicino la masseria di Centrone e di Montaltino. Il dottor Casale lo rinvenne nelle prateric della masseria denominata scannagallina.

Thyuos capitatus. Questa aromatica pianta, o piccolo frutice che dir vogliamo, cresce abbondante nelle più lontane contrade del tratturo. Fu un tempo in cui copriva massima parte de' pascoli, e porgeva largo cibo alle api, la di cui industria era allora più ricca nel tenimento barlettano. Estesa sommamente la dissodazione de'terreni per piantar vigne (!!!), oliveti, e mandorleti, mancò il nutrimento copioso alle api, e l'industria vi si scemò. Aggiungete lo sterminio che ne fanno le contadine per uso di combustibile, ed ecco perchè la pianta diminuisce assai nel numero dei suoi individui, che certamente frutlavano prima più colle api che non rendono adesso qual materiale da bruciare. $\mathbf{E}$ le vigue? Attendete pazientemente che coll'andar degli anni finisca la malattia dell' uva, e poi le osserverete col teloscopio di Henscues per poterle distinguere nella loro ligura rappresentata da un' altaléna, sulla quale leggerete: $i$ padri si arricchiscono, $i$ figli s'impoveriscono.!!.. 
Linum Conymbulosum. Abita nei terreni erbosi e marnosi a bacio poco lungi dal castello.

Viscum album. La parola vernacola del Viscum è originale in bocca de' potatori che lo chiamano màmma dell'albero, quasi dir volessero che la parassita fa da madre e l'albero fa da figlio. Il vischio bianco l'ho raccolto sempre su i mandorli nelle campagne di Barletta, di Capurso, Gioja, Montrone, Trani, ed in altri luoghi delia Provincia di Bari; mentre non l'ho rinvenuto mai sul sorbo, quantunque quest'albero vi si coltivi. I sorbi poi alle falde del Vesuvio e dell' eremitaggio del Salvatore sono stati da me trovati sempre carichi del vischio, ed i castagni annosi di Monteforte nel gennajo 1856 (di felice memoria!) erano pieni della parassita che mi fu impossibile raccogliere perchè vegetava su pei rami che formavano la parte la più elevata della chioma de' castagni, ed il terreno, dal canto suo, era coperto da due palmi di neve. I mandorli che nelle campagne di Barletta ne sono attaccati, soffrono immensamente, per lo più seccano, o menano avanti una vegetazione stentata in tutti i rami su i quali cresce il vischio. Non ci è altro rimedio che tagliarli immediatamente quando apparisce il malefico vegetabile. Il sig. Bors-sIEn riferisce cone sugli olivi della Palestina e del Sud della Spagna cresce un'altra specie di vischio, cioè il $V_{i-}$ scum cruciatum, il quale, avuto riguardo alla latitudine ove son posti la Palestina ed il Sud della Spagna, dev' essere pianta ch' esige una temperatura più elevata di quella di cui si godono gli oliveti del nostro Regno. Avrei volu- 
to su di ció consultare la bella opera del celebre Agronomo sig. De Herrera, ma siccome non conosco la lingua spagnuola, nè me n’è venuta mai per le mani la traduzione sia in francese sia in italiano, cosi non bo potuto estinguere la mia curiosità per sapere, se il Viscum cruciatum arrechi poco o grave danno agli olivi di quelle regioni, e quale pratica usino i coltivatori di colà per distruggerlo. Pregai in aprile corrente anno 1857 la bontà del sig. Gussone circa le notizie di questa parassita nella Spagna, ed egli mi disse che non l'ha mai veduta. Notate bene, o Lettore, che se quegli olivi ne sono assai danneggiati, è giuocoforza che gli agricoltori si abbiano un'altra cura di più nella coltivazione degli alberi di Minerva; cura della quale noi fortunatamente non abbiamo bisogno.

Caprificus ..... ? Io non ho riportato qui il nome specifico del caprifico, poichè di tutte tre le varietà che vegetano nelle campagne barlettane non so precisamente quale di esse corrisponde a quelle specie di caprifico dottamente descritte dal valentissimo Botanico sig. Gasparnixi nella sua pregiata Memoria sulla caprificazione. Nella sudetta Memoria furono riferite dal Professore quelle notizie sul caprifico e sul cosi detto fico caniglioso che nel $1844 \mathrm{gl}$ 'inviai da Barletta in Napoli. Delle tre succennate varietà una addimandasi prufico biancolillo: l'altra dicesi prufico vallarano o vaddarano: la terza prufico nero. La prima e la seconda sono bianche, l'ultima è bruna. Quella denominata vallarano porta il peduncolo lungo tale quale il cosi detto fico vallarano che somiglia un poco al trojano de' Napolitani, le altre due hanno corto peduncolo. 
Ficus carica sulvatica. Il fico nasce in molti luoghi aridi, calcari, sassosi, tufacei, ed anche sulle macerie e sulle fabbriche antiche e quasi dirute. Pel fico cade qui la stessa osservazione e sospetti che proposi più innanzi parlando della vite. Sono forse gli attuali fichi selvaggi avanzi degli antichi ficheti coltivati? Sono aborigèni? Sono effetti degli uccelli che ne depongono i semi o granelli non digeriti nei loro visceri? Sono opera involontaria della mano dell' uomo? In quanto agli uccelli io ne veggo tutte le probabilità. Cade intanto acconcio il dire alcun che sulle varietà di fico selvaggio che s'imprendono a coltivare. A Bari e a Giovinazzo ho mangiato fichi selvaggi coltivati appositamente nei giardini. I loro frutti erano grossi, bianchi al di fuori, rosei al di dentro, di sapore alquanto acidetto assai gradito al palato. A Giovinazzo nel 1851 impresi a coltivarne uno nato tra le fabbriche di una gran vasca di acqua dolce. Lo trapiantai in buon terreno, e nel primo anno portò molti fioroni (volgarmente colombri, culumbri, palasciani) che se ne caddero prima di naturare. I suoi secondi frutti autunnali erano bianchi al di fuori e rossi nell' interno; il sapore alquanto scipito. A Barletta si coltiva una varietà di fico, denominata fico di Santa Maria. E precoce a maturare. Il meritallo tra una foglia ed un'altra è assai lungo. Le foglie piuttosto piccole si sviluppano e ruvidissime. Porta frutti di color pavonazzo al di fuori, biancastri al di dentro con polpa poco succulenta e rari granelli. Però i fioroni sono assai gentili e saporosi, ma non raggiungono la squisitezza di quelli del fico vernino. La scorza è ruvida senza pari. Il peduncolo è molto allun- 
gato. I frutti autunnali, o secondi fichi, se non sono perfettamente maturi, eccedono in succo lattiginoso assai più delle altre varietà di fico, ed è perciò che a Giovinazzo vien chiamato giustamente fico lattarolo. L'abito e il portamento dell'albero ha molto del selvatico; e se il terreno, ove cresce, non è fertile e alquanto fresco, i frutti addivengono quasi sempre piccoli, di poco pregio, e di rustico aspetto. Chi ci assicura che siffatta varieta non siasi da qualche tempo presa a coltivare dallo stato silvestre in cui forse cresceva?

Fraxinus excelsior. Il frassino cresce spontaneo lungo le sponde dell' Ofanto, ove spiega una vegetazione maestosa ed imponente in quei terreni la di cui profondità raggiunge $\mathrm{i}$ venti, i trenta, e quaranta palmi. La chioma dei suoi rami è con tal pompa spiegata, che ne invita a goder dell'ombra nel fitto meriggio. La sua presenza in quelle contrade dimostra che qualche raggio della zona vegetale garganica si distende fino in quei luoghi. Non sarebbe utile impresa pei possidenti quella di estrarne la manna come fanno sul monte Gargàno? Basterebbe sperimentarne le prime incisioni in luglio ed in agosto per assicurarsi della convenienza di questa bella industria. Fa d'uopo di pochissimo spesato, cioè di sei in dieci carlini, e di un'aura di pazienza e d'istruzione: su di che coloro $i$ quali amassero principii istruttivi, possono leggere l'opera del Padre Manicone stampata nel 1806 e quelle degli Agrononi Siciliani che ne trattano con tutta maestria e dottrina.

Olea europaea oleaster. L'olivo selvaggio abita in 
molti luoghi incolti, siano sassosi e aridi, siano feraci nelle vicinanze del fiume. I pastori, i massari, e tutti gli altri coloni l'addimandano trièmeto (pronunziate muta la seconda e). Chi può pescare il significato di questa voce che sia allusiva all'olivo selvatico? I proprietarii ne prendono tutta la cura là ove nasce; poichè giunto ad un certo sviluppo lo trapiantano in buon terreno e poi innestano, come più avanti dissi. Sopra fatti di tal natura è poggiata la estesa industria, sul monte Gargàno, delle gambette di olivo che si diffondono per quasi tutti i paesi olivetati delle due Provincie di Bari e Foggia. A proposito dell' olivo, dal selvaggio passando al gentile, permettetemi una digressioncina, ed eccola quà. Non mi sovviene bene, se nel 1850, o 1851, io inviai da Giovinazzo in Napoli ai due valenti Zoologi, Padre e Figlio Signori Costa, taluni rami di olivo i quali erano bucherati da vermini. Dopo qualche tempo ricevei in Giovinazzo una lettera dai sudetti CosTa, i quali mi dimandavano varie notizie intorno ai rami di olivo da me ad essi inviati, e precisamente amavano conoscere in quale epoca io li aveva raccolti. Risposi subito ai Professori, indicando loro tutte quelle cosette che meglio potevano soddisfare gl'interessi della Scienza. In questo anno 1857 essi mi facevano premure per avere dalla Puglia altri rami di olivo simili ai primi. Io scrissi subito a Barletta e a Molfetta : e di fatti dalla prima Città mio Fratello me ne inviò diversi, tra i quali i Signori Costa trovarono qualche traccia delle uova dell' insetto simile a quello da essi rinvenuto nei rami avuti da Giovinazzo, anni or sono; e dalla seconda Città il sig. D. Mauretto 
De Judicibus ne spediva degli altri che mi feci un pregio presentar subito ai Professori, i quali ebbero l' amabilità di farmi osservare le figure di un insetto ch' essi tengono quasi per certo come un genere tutto nuovo. Ecco dunque quali sono gli effetti dello studio e della passione alle Scienze. Il loro progresso a gradi a gradi si avanza quando ci è tutto l'amore a raccogliere e ad osservare i fatti naturali, in vece di perder tempo alla lettura de' romanzi spurii e di altre produzioni leggiere e di nessun valore!!

Pulllyraea medu. Le foglie di questa specie somigliano a prima vista un pochettino nel colorito e nella larghezza a quelle del Rhamnus alaternus, ed è facile che il suo nome vernacolo latierno tragga origine guasta dal latino alatermus. Negli erbaggi dell' Ofanto vi sono macchieti colossali di Phillyraea frammista al Rovo, col quale forma barriere insormontabili tra un parco ed un altro: e siccome havvi del pari il Rhamnus alatermus, così è naturale il supporre che i contadini ed i mandriani confondano l'una pianta coll'altra.

Orobanche prdinosa. I campagnuoli ed i cọloni delle masserie riconoscono le Orobanche col nome di sporchia, e distinguono la sporchia masculina e la sporchia fomminina. La prima, secondo la loro maniera di vedere, è quella che cresce a ceppaja: la seconda poi resta sempre isolata, cioè ad un sol fusto. Vale a dire che lo sviluppo a ceppaja si osserva da che nasce finchè fruttifica la pianta, ed il contrario si realizza quando cresce ad un solo stelo. Io mi ricordo di aver visto più volte la diversità del portamento tra la sporchia masculina e la sporchia fem- 
minina, ma la brevità del tempo, durante le mie perlustrazioni botaniche ed agronomiche, non mi ha mai permesso di raccoglierne tutti i particolari. Ora io domando ai Botanici: sarả questo fatto una varietà della stessa specie? sarà forse effetto del terreno? sarà effetto diverso del principio fisiologico circa l'influenza nociva delle Orobanche sulle leguminose? Me ne ricordo due altre specie, tutte due piccole, cioè a fusto corto e a piccola corolla: una color paglino, e l'altra color violaceo. Queste due Orobanche le ho viste sempre nei terreni calcari e sassosi; mai negli argillosi come la prima. Le località istesse piene di sassi e pietre fanno si che $\mathrm{i}$ coloni mai o rarissimamente pongono legumi. E comechè la mia premura per la conoscenza di queste parassite è slata sempre dal lato agrario, cosi considerandole nei luoghi, petrosi come piante indifferenti rimpetto ai legumi che mai vi si coltivano, non mi sono affrettato in veruna occasione a raccoglierne i saggi, per indi ottenerne i corrispondenti nomi specifici dalla cortesia del sig. Gussone.

Papaver Rhoeas. Nel linguaggio popolare la parola squàcquera significa cosa molto aperta. E siccome la corolla assai distesa di questa specie di papavero ne dà un esempio chiaro, cosi tutte le classi di persone chiamano squàcquera il Papaver Rhoeas. I vigneti, i seminati, gli oliveti ne abbondano da per ogni dove. Ecco, secondo DE Candolle un altro esempio des plantes cultivées malgrè la volonté de l'homme. La Matricaria chamomilla, lo Cnicus arvensis e tante altre piante tutte proprie di quelle campagne, andrebbero ben classificate in questa categoria. 
Anagyris foetida. I mandriani chiamano questo frutice piseddo di scorzone e lupino salvaggio. La parola scorzone presso il basso popolo significa quella specie di serpe comune e di colore bruno: ora che ha che fare la serpe colla pianta ch'è piuttosto velenosa? Forse le serpi si cibano dei suoi frutti?

GlycyrRhiza glabra. Questa pianta industriale cresce alle paludi verso l'Est, e vicino le foci dell' Ofanto all'Ovest della Città nei terreni acquitrinosi e salini. Ivi le coltivazioni di altre piante non pruovano bene se prima coll'andar degli anni non vi si diminuisce la soverchia salsedine, coltivando ed ingrassando sempre: ecco perchè grandi estensioni di quei terreni restano continuamente incolte. In questo caso io domando: e non sarebbe miglior partito diffondere la moltiplicazione della liquirizia, che vi è spontanea, ed ivi stabilirne una fabbrica, allo stesso modo che si fa nella Provincia di Foggia, nella Basilicata $e$ in altri luoghi del Regno?

Hedysarom echinatum. Ecco una bella specie di leguminosa perenne, eccellente per foraggio ad ogni razza di animali. Somiglia un poco alla lupinella, e trovasi rara nei pascoli aprici del tratturo nelle vicinanze di Montaltino, e presso i colli di Canne, ove la raccolsi nel 1847.

Medicago scutellata. I frutti o baccelli delle erbe mediche essendo nella massima parte disposti a spira, siffatta conformazione pare che abbia data origine alla voce volgare ciurufuogghio femminino, che significa cosa attoreigliata. Il chiarissimo signor Gossone sempre mi ha suggerito che le erbe mediche non si possono ben distinguere 
nelle loro specie, se prima i rispettivi baccelli non siano totalmente sviluppati: lo che si osserva in aprile ed in maggio, secondo la precocità della specie, la qualità dei terreni, e dei luoghi, ed il corso della stessa stagione.

Medicago lappacea et Medicago Histrix. I campagnuoli le addimandano col nome ciurufuogghio masculino. Come pescare l'idea di relazione di maschio e di femmina tra queste specie diverse, secondo la maniera di pensare de’ coloni? Sarà forse la conformazione de' frutti, o la loro vestitura che ha data origine a queste voci?

Menicago coronata. Quando io inviava da Barlella a Napoli al sig. Gussone i saggi delle piante, egli in una delle sue lettere mi onorava delle seguenti parole: Mi ha fatto piacere trovare in fra $i$ saggi delle vostre erbe mediche uno della bella Medicago coronata. E veramente i frutticini di questa specie nella loro conformazione e struttura or ganica somigliano ad una piccola corona. Due volte è stata da me rinvenuta nei pascoli aprici di Montaltino, ed in quelli che stanno tra la citta di Trani e di Corato, nel 1840. E una specie piultosto rara nel tenimento barulense.

Medicago falcata. I coloni l'addimandano ruveddasce$n a$ : (pronunziate mula la $e$ ) cosa intenderanno con questa parola? d'onde la sua origine? A Giovinazzo la chiamano niervo, a cagione della sua grossa e lunga radice. Questa preziosa pianta trovasi facilmente lungo le strade e nei campi. L'ho raccolta nei seminati, e nei vigneti, sassosi, argillosi, e marnosi calcari: mai l'ho rinvenuta nei silicei. Il signor D. Domenico Tavarone, di Lanciano negli Ab- 
bruzzi, me ne favori dei saggi in novembre 1856 , che portò da quella Provincia. Come specic perenne che vegeta in piena state sino a due palmi e più di altezza, senza bisogno di acqua, è un tesoro per gli animali. In taluni paesi del Regno l'Ononis spinosa vien chiamata arresta bovi a cagione delle sue spine. Nelle campagne poi di Lanciano la Medicago falcala similmente dicesi arresti bovi, e ciò dipende dal perchè quegli animali mangiandola avidamente, vi si fermano per qualche tempo. Il sig. Gussone sempre ha insistito sulla utilità di essa nei prati artificiali, ed io ad esempio suo non ho mancato di raccomandarla ai proprietarii di praterie. Inutili sforzi.!! . . Nel 1852 ne seminai una piccola quantità a Giovinazzo, e le piante svilupparono bene; ma nel $\mathbf{1 8 5 3}$ avendo dovuto seguire altra destinazione a Cardito, non potei vederne il risultamento. Premura era la mia di moltiplicarne la semenza e distribuirla alle Società economiche.

Melilotus ledcantma. Questa leguminosa cresce colossale e tutta ramosa sino a cinque palmi di altezza vicino il fiume, e gli animali l'appetiscono con avidità. Non pare che sia propria una pianta pugliese, avuto riguardo alla secchezza del clima. In nessun'altra campagna barulense l'ho trovata, meno che nelle vicinanze dell'Ofanto: alle paludi non si trova e molto meno alla contrada di casavecchia ove l'acqua ristagna per buona parte dell'anno in varii punti che sono $\mathrm{i}$ più bassi di quei terreni avvallati. Io son di avviso che la sua presenza, lungo il fiume, sia dovuta ai semi trasportati dalle acque provenienti dalle montague di Santangelo de'Lombardi nella Provincia di 
Avellino. Ed in effetti ama siffatta pianta terreni sciolti e piuttosto umidi e freschi. L'ho raccolta più volte sulla grotta di Pozzuoli nelle vicinanze della tomba di Virgilio: in autunno 1856 la rinvenni a Baja; e nell'ottobre 1855 la trovai fiorita in fra le alture e avvallamenti di Monteforte. Ora quelle montagne fanno parte degli Appennini nostri che si continuano in più rami per varie direzioni, e conseguentemente le loro circostanze fisiche locali saranno presso a poco simili a quelle ove ha origine l' Ofanto nelle vicinanze di Santangelo dei Lombardi, d'onde i semi portati dalle acque, come più avanti ho detto, fanno apparire la pianta in contrada a lei estranea, qual'è la Puglia.

Onobrychis caputgalli. Abita nelle anfrattuosità de’terreni aridi nelle contrade del tratturo, ove abbonda il tufo ed il carbonato calcare sassoso. Una prateria artificiale di questa preziosissima leguminosa costerebbe piccolo spesato; giacchè non si tratta di zappare il fondo a regola d'arte, benvero di smuovere il terreno alla profondità di un quarto di palmo or quà or là: vale a dire che il lavoro della zappa non dev' essere continuato ed in massa, piuttosto a salti, cioè ogni due o tre palmi mezzo colpo di ferro. Coloro i quali conoscono a fondo la natura della lupinella e cosa significa prateria mista, comprendono subito il perchè di questa regola eccezionale ed economica. Noi ci auguriamo che i proprietarii si destino un poco da tanta lentezza d'industria per quei terreni sassosi destinati alla pastura delle povere pecore che in certi mesi dell' anno sono condannate a cibarsi dell' amara frasca di olivo, che non è certamente il più bel foraggio del mondo! Al- 
ternarla colla lupinella, col fieno, e con altro foraggio fresco, sarebbe un vantaggio immenso per le greggi, che alla bontà delle carni e delle lane accoppierebbero una migliore produzione di latte nella quantità e nella qualità.

Trifolium frigiferdi. Lungo le rive del fiume, ove le praterie naturali sono superbe (e più lo sarebbero, se fossero sgravate di tante piantacce inutili e nocive), vidi nel maggio 1847 un parco di prateria, ove l'abbondanza del Trifolium fragiferum spontaneo costituiva i quattro quinti della estensione del terreno con una floridezza di vegetazione veramente degna di essere ammirata. Gli è proprietà de'signori De Leone, i di cui latticinii sono de' più rinomati per la loro squisitezza. Come pianta perenne il Trifolium fragiferum forma la ricchezza di quegli erbaggi. Associato gli era il Trifolium flavescens biennale, ed altre specie di trifogli annuali.

Vicia grandiflora. Coloro che si dànno allo studio della Botanica conoscono che questa specie è spontanea nelle selve di castagno ai Camaldoli, Lago di Agnano, Valle di San Rocco, Capodimonte, all' eremitaggio del Salvatore, a Castellammare e luoghi simili, ove l'ho raccolta più volte. E questo fatto dimostra che la pianta ama terreni umidi, freschi, ed ombrosi. Il sig. Gussone si compiacque quando gli presentai i saggi raccolti nell' agro barulense nel 1847, avuto riguardo alla località pugliese il di cui suolo è arido e nimico del castagno. Abita nei luoghi a bacio dirimpetto al mare, vicino al castello. Un'altra specie di Vicia, diversissima dalle altre, rinvenni nei campi. Sventuratamente un solo sagrgio raccolto, e mesco- 
lato a tante altre piante, non mi fu possibile rintracciarlo per poterlo disséccare e farmene indicare il nome specifico dal prelodato Botanico. Quindi ho lasciato in bianco il suo posto nell'elenco a pagina 99 , per ricordarmi le ricerche a farsene in migliori occasioni.

Plantago langeolata varietas altissima. Questa bella varietà di piantaggine l' ho sempre raccolta nei così detli vigneti di Trojaniello; e non ho avuto mai l'opportunità di osservare se gli animali l'appetiscono con piacere. Se i suoi caratteri fitognostici sono costanti ab origine, e non sono l'effetto della coltivazione involontaria dell' uomo mercè $\mathrm{i}$ lavori che si fanno alle vigne, è chiaro il comprendere esser dessa una varietà pregiatissima pel suo fogliame copioso, lungo, e largo. Di tal che una prateria, che ne fosse piena, somministrerebbe copia di foraggio agli animali assai maggiore di quella che si ottiene dal tipo della specie. Se non che meriterebbe calcolarsi il valor nutritivo di siffatta varietà, e quindi farne lo studio conparato con quello della vera Plantago lanceolata. Se leggete, o Lettore, gli specchietti esposti nell'opera del grande Maliguti, del sig. Grananin, e di altri Chinici agrarii, vedrete quanto sono indispensabili le cognizioni di questo genere, e quanta sia grande la utilità rispettiva!

Plantago albicans. Vegeta nei terreni calcari lufosi e marnosi i più secchi del tratturo.

Statice caspia. In una delle sue letlere a me direlle in Barletla il chiarissimo sig. Gussone cosi si esprimeva: Vi ringrazio de'saggi della bella Stalice caspia. Abita nei siti argillosi per eccellenza alla fumara in mezzo alle Salicor- 
nie, agli Atriplex, alla Statice limonium, e alla Statice serotina. Alle paludi non l'ho trovata mai.

Statice nubia. Cresce nei terreni marnosi a bacio dirimpetto il mare all'Est e all'Ovest della Città.

Sratice...... ? Se io fossi un Botanico, la chiamerei Statice barulensis. Nel mese di maggio 1847 ne raccolsi taluni saggi in piena fioritura vicino le siepi di Atriplex Halimus che circondano la masseria di Santo Lazzaro, e propriamente dalla parte interna, lungo il canale di difesa in terreno argilloso e pantanoso. Era una pianta unica e sola fiorita, e tutte le altre non davano nessun segno di vegretazione. Ivi abbondano a centinaja di migliaja le piante della Statice limonium e Statice serotina, e cominciano a fiorire verso il cadere del mese di luglio. Il suo portamento, il fogliame, la ramificazione, la disposizione dei fiorellini, e lo stesso colorito: tutti questi fatti la facevano distinguere dalle altre specie che io aveva raccolte nell'autunno degli anni antecedenti 1837-1838-1840-18411842-1844. L'egregio cavalier Gussone la vide, e mi favori grentilmente dirmi che se ne sarebbe occupato a studiarla. Io ne aveva solamente due saggi, de' quali uno per lui e l'altro per me. La particolarita della fioritura, cento giorni prima delle altre piante, eccito i miei sospetti che si fosse un'altra specie o varietà positiva. Sul Giornale il Lucifero ne pubblicai un cenno: Anno $100^{\circ} \mathrm{N} .{ }^{\circ} 390^{\circ}$ li otto dicembre 1847.

Rumex conglomeratus. Il nome vernacolo di questa pianta è curiosissimo: schialla pignatte. E probabile che Lrarrà origine da qualche fatlo posilivo, per cui i co- 
loni e il basso popolo riconoscono la specie con queste voci.

Ronex patientia. Abita nei fossati, nei canali, ai margini de'bassi fondi, e nei terreni argillosi marnosi nelle vicinanze delle foci del fiume.

Rumex tirrsoldes. Raccolsi questa specie nel maggio 1847 sugli aprici colli di Canne ove cresce bella pastura per le pecore.

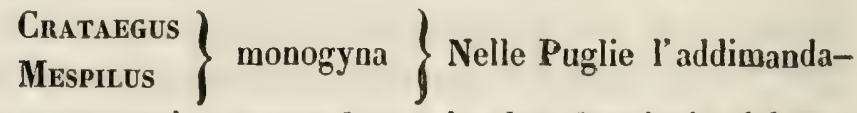
no scarapurcio, scarapulce, e in altre Provincie del Regno spinapulce. Vi è dunque una certa analogia tra questa voce e quella del colono pugliese. I nomi volgari delle piante cambiano in bocca de'contadini di uno stesso paese, e variano da un paese all'altro: mentre in questo caso del bianco spino (aubépine) il nome vernacolo è quasi simile e lo stesso in molte Provincie. D'onde questa analogia di voci allusive alla stessa specie? Le sponde dell'Ofanto sono cariche del bianco spino, che trovasi anche in altri siti, benchè aridi e sassosi. Ed io ne ho visto soventi fiate una varietà con frutto grosso due volte quanto un cece. Quei luoghi sono abbandonati alla natura per uso di erbaggi, e conseguentemente non si coltivano. Se fosse causa di questo fatto la sola feracilà del terreno, dovrebbe la grossezza del frutto manifestarsi in tutte le piante; ma io spesso ho ben osservate le piante di bianco spino vicine tra loro, delle quali talune a grosso frutto con foglie larghe e quasi lobate, ed altre con frutti piccoli, a strette foglie incise e con lacinie acute. D'onde proviene 
dunque questa grossezza di frutto? Sara forse una distinta varietà?

Pynos cuneifolis. Tutti i campagnuoli ed i massari l'addimandano perazzo. Siffatta parola esprime assai bene lo stato selvatico e ruvido dell'albero. Le praterie in molti luoghi sono coperte di peri selvaggi ; e v'ha de'possidenti, i quali, senz' altra coltura, gl'innestano, e ne ottengono belle e abbondanti pere. Nell'agosto 1844 ne vidi numerosi esempii ne'territorii di Canosa, e fra le altre varietà mi si mostrò una, denominata pera del sole, di colore rosso paglino, e della grossezza di quella chiamata pera del carpio: l'era dessa pregiata pel tenerume della polpa, pel sapore e fragranza. Nella masseria de'signori Esperti e di altri Barlettani si sono fatti bellissimi esperimenti su questa novella industria, ed i fatti sono stati coronati da felicissimo successo, i quali dimostrano esattamente la breve e semplicissima teoria stabilita dagli Agronomi; cioè, che la pianta gentile moltiplicata per mezzo dell' innesto, può prosperare in qualunque terreno, purchè inserita sopra altra pianta della stessa razza. La teoria ̀̀ piuttosto moderna, il fatto e la verità sua sono cose antiche quanto la stessa Natura! Mi si permetta qui citare poche parole di Varrone, il quale diceva cosi : Se sul pero selvaggio porrai pero gentile, avrai buono il frutto; e se sul primo gentile ne porrai un secondo, allora il frutto sara gentilissimo. Ora se un curioso dimandasse: d'onde proviene la gentilezza dei frutti in generale? si direbbe : dalla coltivazione continuata in buon terreno e sotto favorevole cielo, sic et simpliciter: e se di più interrogasse: ov' è la 
origine della coltivazione? si risponderebbe: DANS LES CAvES DE L'OBSCURITÉ DES SIÈCLES!!

Delpinnum longipes. Ecco un' altra specie, fra tanti vegetabili, cultivée malgré la volonté de l'homme. Nasce da per ogni dove, ed anche nei seminati. Nel tempo della mictitura molti saggi, sfuggiti alla mano del coltivatore, e con semi maturi non ancora caduti dalla pianta madre, vanno mescolati col grano. Quindi ne segue che nel trebbiarsi il frumento, le semenze del Delphinium longipes restano confuse con quelle de'cereali, e sono riconosciute dal basso popolo colle parole occhio di pupa. Vedete a proposito quanto ho detto sulla Lychnis Githago.

Ranunculus bullatus. Abita nelle praterie secche al Sud-Est della Città. Lo raccolsi in novembre $\mathbf{1 8 4 0}$ nelle vicinanze della masseria di Barracchia alla contrada di Santo Luca. Nel novembre 1850 lo rinvenni sulle parieti a secco, coperte di terreno calcare, tra Giovinazzo e Bitonto; grazie alle gentilezze del defunto Economo dell'Ospizio di Giovinazzo, il quale m' invilò ad una passeggiata campestre sino alla citta di Bitonto. Fu in quella oceasione in cui raccolsi diverse piante, che inviai subito al signor Gussone. La Clematis cirrosa non isfuggi alle mie ricerche; ed i saggi raccolti dai pochi macchieti (rabougris) dell' Ulmus campestris, e dai floridi alberi della Celtis australis, mi fecero noto che queste due piante arboree erano ivi coltivate un tempo, in cui gli antichi padroni di quei tenimenti si avevano un boschetto sia per diporto, sia per uccellare, o per altra causa. L'olmo è facile che vi fosse spontaneo, perchè mi ricordo benissimo sin dal 
1831, che quando percorreva i miei primi studii lelterarii nel seminario di Trani, quelle campagne mi offrivano di tinto in tanto macchicti di olmo in terreno calcare e sassoso, carichi di quelle vesciche prodolte dalla puntura degl' insetti, per lo che i nativi di quei luoghi l'addimandavano albero delle mosche: ed il terreno di Bitonto e di Trani è quasi del tutto lo stesso, calcare e sassoso come nella maggior parte della Provincia di Bari. Ma in quanto alla Celtis australis, certo è da ritenersi che i pochi e robusti alberi da me osservati, avanzi fossero di passata collivazione.

Thalictrum nigricans. Nasce florido nelle praterie dell'Ofanto poco lungi dai colli di Canne.

Poteriun garganicum. Abita nei pascoli aprici i più lontani dalla Città nei siti elevati del tratturo. Sarebbe questo un altro esempio, come quello del Frassino, per riconoscere la estensione fin là di qualche raggio della zona vegetabile del monte Gargàno? Vedete quanto ne accennai sul Frassino.

Asperula crnanchica. Vegeta nei terreni calcari lungo la via vicino il convento de' Cappuccini: e convive colla Gypsophyla permixta e colla Scabiosa grandiflora prolifera.

Peganum harmala. L'abitazione di questa pianta è circoscritta nei pascoli del tratturo, e propriamente in quella zona pratense, la quale è posta al Sud-Ovest di Barletta; e la si rinviene nei prati vicino il crollato ponte del fiume, ed in quelli che marcano il limite tra i confini de'due territorii di Barletta e di Canosa. Più l'osservatore si avvicina a Canosa, e più la trova abbondante; finchè pro- 
gredendo verso Cirignola e Foggia, la rinviene padrona de' prati. Nei pascoli tra Canosa ed Andria, e quelli di Molfetta, Corato, Ruvo, e Terlizzi non l'ho vista mai. (Con ciò non voglio sostenere che ivi non vegetasse). Ecco il vero tipo della vegetazione del Tavoliere di Puglia. Singolare coincidenza! Taluni contadini la chiamano ruta salvaggia, sed i Botanici la classificano nella famiglia delle $R u$ taceae. Dice benissimo il sig. De Candolle (figlio) che i caratteri i più rilevanti e specchiati nelle piante si riconoscono facilmente anche dalla gente di campagna che non intende al certo lo studio scientifico.

Ruta bracteosa? La raccolsi nei terreni tufosi nelle vicinanze delle masserie Centrone e Passero. Devesi ritenere cume veramente spontanea, o quale avanzo di antica coltivazione? Sulle antiche fabbriche del castello di Trani vidi (non mi ricordo in quale anno, se nel 1840 o nel 1841 ) molte piante di Ruta che prosperavano nelle fenditure di quell' edificio.

Trinulus terrestrus. Vegetabile molesto! Ed a ragione gli ortolani lo chiamano vasa piedi, perchè si conficcano i suoi frutti spinosi nei piedi de'coltivatori che vanno scalzi ne' momenti di lavoro e nelle giornate calde; ed è così che le punture si rendono più dispiacevoli.

Thesium humle. Rarissimamente si trova questa specie nelle campagne di Barletta. Abita vicino il tratturo dirimpetto la masseria Centrone ed il casino Passero, e propriamente in quel sito ove il suolo abbassandosi dolcemente in mezzo ad amene piagge, dà luogo ad una corrente di acqua durante le strabocchevoli piogge d'inverno. Ivi il 
lerreno è siliceo-calcareo-argilloso ed in altri punti è siliceo-argilloso-calcare. Nel Maggio 1847 ne raccolsi per la prima ed unica volta un solo saggio. ed era in fruttificazione. Vedete quanto ne dissi nella descrizione topografica delle campagne di Barletta.

Bartsia trixago. Questa pianta annuale fiorisce in maggio, ed invade oltremodo $i$ seminati a grano, e ne arreca danno. Nella Provincia di Foggia pare che la confondano col Melampyrum arvense, ed ivi la chiamano sporchia di grano, poichè il male che apporta ai cereali si assomiglia nell'intensità a quello che le Orobanche fanno alle civaje. (Vedete l'elenco de' nomi volgari alla parola sporchia). Sarebbe necessario sperimentare nei campi se il male prodotto dalla Bartsia, sia effetto della facoltà assorbente delle sue radici, superiore a quella delle radici de' cereali, ed in quale stadio di vegetazione, se nel primo, o nel secondo : o diversamente, se siffatto nocumento generato sia dalla innumerevole quantità delle piante di Bartsia: o se in fine dall'essere questa specie una vera parassita. Sono simiglianti studii assai delicati, ed esigenti pazienza e costanza. Vedete quel che ne scrissi sul giornale la Rivista agronomica.

Linaria triphylla. Cresce nei seminati vicino il convento de Cappuccini: e non l' ho rinvenuta mai in altri fondi dell' agro barulense. E spontanea altresi nei terreni sotto marnosi di Giovinazzo, e precisamente nel fondo denominato la cocévola nelle vicinanze dell'Ospizio (1). Questa

(1) Gli ortolani Giovinazzesi per cocévola (pronunziate breve la sillaba vo) intendono ogni terreno destinato alla coltura degli ortag- 
graziosa specie l'ho vista più volte negli anni 1835-1836 e 1855 collivata nelle Ville alla Barra presso Napoli.

Linaria volgaris. Nei seminati e nei vigneli della contrada di Trojaniello copiosamente si rinviene. Nel $1854 \mathrm{e}$ 1855 l'ho raccolta vicino le siepi nel tenimento di Cardito di Caivano, e propriamente dirimpetto il basso fondo denominato la taglia, ove negli andati tempi erano numerosi alberi da frutto, che furono poscia tagliati, d'onde ebbe origine la parola taglia.

Linaria minor. Vegeta nei depositi di terre silicee, prodolti dalle acque dell'Ofanto lungo le sue sponde.

Verbascum nepandum. Abita nei terreni argillosi ed umidi de' bassi fondi alla contrada denominata casavccchia.

Datura stramonium. Lo stramonio per la feracilà estrema del terreno lungo le sponde dell' Ofanto raggiunge l'allezza di sei palmi, con grosso fusto assai ramoso che sembra un alberetto, portante ampio fogliame e grandi fiori. Alla via del campo, vicino Napoli, dal 1835 sino al 1856 i saggi di questa pianta medicinale li ho raccolti fioriti e sempre dell' altezza di mezzo palmo, aventi i fiori piccoli e foglie meschine. Ai Bagnoli poi e all' estremità di $P_{0-}$ silipo l'altezza delle piante è stata da me misurata sino a tre palmi. Lo studio comparato in tutte le Scienze è indispensabile; ed in quello della Botanica è indispensabilissimo, quando, avuto riguardo alla generalità delle piante

gi, come quello il quale essendo necessariamente coltivato meglio e spesso ingrassato, più di leggieri si presta alla buona produzione di siffatte piante, rendendole di facile cottura. 
coltivate, se ne voglia fare l'applicazione in Agricoltura sotto cinque rapporti: $10^{\circ}$ il clima: $2 .^{\circ}$ il terreno: $3 .^{\circ}$ la posizione topografica del luogo: $40^{\circ}$ la coltivazione: $50^{\circ}$ e la forza regetativa dell' individuo della specie, la quale forza è in ragion composta della particolar natura del vegetabile e delle quattro anzidette condizioni. Queste cose ho voluto quì notare, non perchè ritenessi che lo stramonio sia pianta agraria, ma perchè mi è venuto a proposito di ricordare i sani principii poggiati sopra i fatti e confermati per lungo tempo dall' esperienza degli Agricoltori sennati e dalla dottrina dei Dotti. Principii i quali vorrebbero essere più severamente studiati da coloro che si dàno all' industria campestre!

Hyosciauus albus. Il volgo lo chiama sucaméle perchè i fanciulli dalla estremita inferiore del tubo della corolla ne succhiano il nettario di sapore dolce.

Hyoscianus niger? Mai ho rinvenuto il giusquiamo nero nelle campagne di Barletta; ma il Dottor Casale, mio Zio materno, spesso mi diceva averne raccolte le piante negli erbaggi dell'Ofanto nei primi tempi della sua carriera botanica. Sarebbe a desiderarsi che altri ne facessero ricerche esatte, essendo che è pianta medicinale generalmente usata.

Lyciur europaeur. Vedete quanto ne ho accennato parlando dell' Atriplex Halimus.

Rhus Coriaris. I vignajuoli l'addimandano pistazzieddo. Questo nome vernacolo ha il suo significato, ed ecco. I coloni e tutto il basso popolo riconoscono il frutto del carrubbo colla parola pistazzo; il quale carrubbo ed il som- 
macco portano foglie composte pennate; ed è per una piccola similitudine di questo carattere fitognostico che i con tadini dicono pistazzieddo, quasi dir volessero piccolo carrubbo. Tutti sanno la ricca industria che del sommacco si fa in Sicilia: potrebbero i Pugliesi occuparsene, poichè questa pianta non esige tanto spesato quanto le altre coltivazioni. Cresce spontanea nei vigneti all'Est della Città, e precisamente alla contrada Fraggianni, alla via vecchia dello Sterpeto, e nelle vicinanze della strada interna che conduce alle paludi. Nel 1840 raccolsi il Rhus coriaria presso Trani nei luoghi sassosi e calcari come a Barletta.

Amur majos. Comunemente accitieddo, diminuitivo di àccio, che in bocca del volgo significa sèdano: e siccome le foglie radicali dell' Ammi majus somigliano un poco a quelle del sèdano (perchè tulti due appartengono alla stessa famiglia naturale), così i campagnuoli per esprimere l'idea di piccolo àccio, ossia sèdano piccolo, adoperano il nome vernacolo accitieddo.

Apiou graveolens. L'ho raccolto alle paludi. Giusta le osservazioni del signor DE CANnolle sulle vere piante spontanee e su quelle semplicemente naturalizzate, sarebbe ivi l'Apium graveolens da considerarsi come indigeno del paese, o proveniente dagli avanzi di antiche coltivazioni, e quindi naturalizzato? Io sarei per la seconda opinione, dal perchè alle paludi spesso $\mathrm{i}$ contadini coltivano diversi ortaggi. Sentiamo cosa dice il Botanico Ginevrino quando parla dei differenli gradi di naturalizzazione delle piante in un paese. a J'appelle complétement naturalisée, " et par abréviation, naturalisée, une espèce qui, n'exi- 
" stant pas aurapavant dans un pays, s'y trouve ensuite " avec tous les caractères des plantes spontanées indigè" nes, c'est-à-dire croissant et se multipliant sans le se" cours de l'homme, se manifestant avec plus ou moins "d'abondance et de régularité dans les stations qui lui " conviennent, et ayant traversé des séries d'années, pen" dant lesquelles le climat a offert des circonstances excep" tionnelles. " Geografia botanica: volume $2{ }^{\circ}$

Cacmnys pungens. Abita nei terreni calcari ed aridi del tratturo. Nessun nome vernacolo ho potuto rintracciare di questa pianta presso i coloni Barlettani. Solamente nel 1844, avendola raccolta anche nelle campagne di Canosa, ivi i contadini me la indicavano colle parole zampino salvaggio. Cosa vorrà significare presso i medesimi questa parola zampino?

Echinopiona tenuifolia. Questa interessante specie fu da me raccolta la prima volta in agosto 1844 nei vigneti di Canosa. Colà i campagnuoli la chiamavano zampino paesano, e mi assicuravano che gli asini ed i cavalli la mangiano volentieri. Tramanda un odore quasi simile a quello del finocchio; e vane furono le mie ricerche per scoprire il significato della parola zampino secondo il linguaggio de' contadini. Nel 1847 poi la rinvenni nei vigneti poco lungi da Montaltino e dalla masseria Centrone e Passero, come pure in quella del sig. Cafiero verso Canosa. Nei primi luoghi ne trovai pochi saggi, cioè circa cinque miglia (se mal non mi ricordo) lontani dal mare. Nella masseria Cafiero i saggi erano più abbondanti, ed ivi la distanza (se non erro) conta sette miglia da Barletta. Nei vigneti 
poi di Canosa la specie è piuttosto comune: e tutti sanno che quella Città dista da Barletta dodici miglia. Pare dunque che la stazione della specie sia mediterranea e non marittima; ed è probabile che sarà più copiosa in individui la sua presenza nelle carnpagne di Minervino. Sarebbe soddisfacente cosa pel Naturalista la verifica di questo fatto, cioè la progressiva e regolare abbondanza degl' individui della specie in ragion diretta della lontananza dal mare. Qui cade in acconcio il notare un altro esempio del principio stabilito da De Candolle, che i caratteri principali e rilevanti delle famiglie naturali non sfuggono neanche agli occhi del volgo, massime dei campagnuoli. Così la Cachrys pungens e l'Echinophora tenuifolia sono due generi diversi della stessa famiglia naturale delle Umbelliferae, e tutti due sono riconosciuti col nome vernacolo zampino. L'origine di questa voce dipenderà forse dalla conformazione e struttura de'frutti ricciuti dell' Echinophora, o dalle punte delle lacinie delle sue foglie? Sentiamo la descrizione fitognostica che ne fa il sig. Gussone nella sua Flora sicula, volume 1. - Aliquando folia tripinnatifida, laciniis plurimis ad primariam petiolorum divisionem cruciatim dispositis; in caulinis vero laciniae remotae, breviores, parum latiores, rigidiores: involucra integra, inermia, radiis subbreviora, patentia, demum saepe deflexa: involucella in fructu recurva, rigida, sed non pungentia. Più appresso poi riporta il sinonimo di Cupani cosi espresso: $\boldsymbol{P} a$ stinaca sylvestris, angustifolia, fructu echinato. H. Cath. p. 163. Sicchè la parola zampino, usata dai contadini, si avvicina un poco alla descrizione botanica per ciò che ri- 
guarda il suo significato allusivo ad una piccola zampa.

Ferula communis. I fusti di questo vegetabile sono adoperati nella costruzione delle arnie per le api, ed in quella di certi mobili rustici che fanno l'uffizio di sedie nelle case coloniche. Ivi siffatte sedie si addimandano freddizze. Questo è uno di quei nomi vernacoli curiosi e senza allusione alcuna ad un qualche significato.

Foeniculum vulgare et piperatum. I rami fioriti di queste due specie soglionsi adoperare nel conciar le cosi delte olive in acqua o in salamoja, onde la loro parte aromatica conciliar potesse una tal quale fragranza al frutto dell' albero di Minerva.

Opopanax chironium vel Pastinaca opopanax. Il suo fusto somiglia un poco a quello della Ferula communis, ed è perciò che vien chiamata fergolazza: e tanto maggiormente in quanto che nei terreni feracissimi sulle rive del fiume la vegetazione erbacea ed arborea in generale è rigogliosa. Nel 1847 mi dicevano molti campagnuoli, che i pescatori onde prendere maggior quantila di pesce nell'Ofanto, mettono la radice dell' Opopanax in un sacco, e poi lo tuffano nell'acqua in quei siti da essi creduti i più frequentati dai pesci, i quali dopo qualche tempo vanno a galla perchè restano storditi dal succo della pianta. Gli è questo un altro vegetabile che infesta oltremodo quelle ricche praterie. Perchè non estirparlo un poco la volta economicamente? Per quante ricerche feci in quei lunghi, $\mathrm{mi}$ fu impossibile riconoscere che gli animali lo mangiassero.

Valerianelda echinata. E un poco rara; e l'ho rinve- 
nuta più volte solamente all' Ovest del convento de' Cappuccini nei terreni argillosi seminati a cereali e a legumi, e precisamente presso il vecchio oliveto.

Vitex agnuscastus. I mandriani, i massari, e i costruttori di panieri e di cesti lo chiamano canapazzo, che significa senza dubbio canapaccio o canape grossolana. Ma quale analogia passerebbe tra il castoagnello ed il canape? Siamo giusti verso i campagnuoli e le loro ristrettissime idee. Le foglie della Cannabis sativa e del Vitex agnuscaslus somigliano sino a un certo punto fra loro. Di più , i fusti dell' agnocasto si adoperano nella costruzione dei panieri; e siccome sono pieghevoli e alquanto tenaci nella scorza, così quella parola capanazzo è tollerabile, quasi che alluder volesse all'idea della tenacità del canape.

Pancratuda maritumum. Abita nei terreni arenosi del mare, alle paludi e alla fumara. Vien riconosciuto colle voci giglio di S. Giacomo. Ecco un altro nome vernacolo di cui non si trova la vera etimologia. Questa pianta bulbosa porta fior bianco che si schiude in agosto; e tramanda odor gratissimo nelle ore serotine. Perchè non coltivarlo nei giardini? Ciò facendo, converrebbe mescolare al terreno buona quantità di arena marittima per la prospera fioritura della pianta. Io l' ho vista fiorita molte volte lungo il littorale fra Castellammare e la Torre Annunziata nella Provincia di Napoli. É dunque una pianta comune sul Tirreno e sull' Adriatico.

Sternbergia lutea vel Amarylus lutea. Si diletla de'terreni calcari e petrosi, ed in particolare relle anfrattuosità de'sassi. L'ho raccolta più fiate alla via di $\mathbf{A n -}$ 
dria, alla contrada del Crocefisso, vicino i vigneti di $\mathrm{Car}-$ done, alla masseria di Barracchia e luoghi simili.

Arom tendifolium. Predilige i terreni erbosi calcari; e l'ho rinvenuto insieme coil' Amaryllis lucea.

Cypenus olivaris. Comunemente l'addimandano erba rossa. In verita nell'epoca della fioritura le squamette o glume dei fiori hanno in massa un colore rossetto gentile, che si rende più pronunziato per quanto la pianta trovasi in luogo esposto al sole ed in terreno ove abbonda l' elemento calcareo: mentre al contrario nei fondi che scarseggiano di siffatto principio terroso e che sono esposti all' ombra, il colore delle glume è sbiadato e biancastro. Il primo falto l'ho osservato spesso vicino l'Ofanto, notando le particolarità chimiche e fisiche del terreno in diversi siti. Il secondo fatto poi l'ho marcato in varii luoghi de' contorni di Napoli, non esclusi i terreni sopra Miradois a fianco della Specola astronomica. I proprietarii de'fondi arenosi a Barletta allorchè li cedono in fitto, fanno la convenzione coi coloni di dover estirpare l' erba ros$s a$, la quale infesta sommamente i terreni, forse più della gramigna, coi suoi rizòmi di facilissimo sviluppo.

Cyperus badius. Chiamasi erba a quadriello. I culmi de'ciperi sono ordinariamente angolosi e triquetri: (Culmi enodes, teretes vel triangulares, basi foliosi: Gussone, Flora Sicula, Volumen primum). Ond'è che sta bene l'espressione dei campagnuoli, presso i quali la parola a quadriello non tanto significa a quattro angoli, quanto un corpo angoloso, quale che esso sia. Qui ricordo nuovamente ciò che dice De Canpolle, che certi dati caratteri 
naturali e chiari delle piante non sfuggono neanche alla vista e all'intelligenza comune della gente di campagna.

Scinpus maritimus. Dicesi parimenti erba a quadriello. Qui si trova la stessa coincidenza di significato nel nome vernacolo. Di fatti il fusto di questa pianta è angoloso triquetro. (Culmo triquetro superne aspero spicisque glabro elc. Gussone, Flora Sicula, Volumen primum).

Colcurcum Cupani. Vegeta nei terreni fertili de' bassi fondi dolcemente avvallati. Nei vigneti di Campanile, poco lungi dal fiume, spesse fiate l'ho rinvenuto fiorito ed in florida vegetazione.

Aegylops ovatı. Questa graminacea vien denominata scanna cavallo. E probabile che le reste lunghe e ruvide della pianta producano danno agli animali cavallini, ed è perciò che i coloni la riconoscono con queste voci. Molti pastori e mandriani mi asserivano questo falto, e mi soggiungevano che in generale gli animali non tanto volentieri l'appetiscono. Sarebbe a desiderare che i possidenti e veterinarii se ne occupassero a studiare queste particolarità. (Spica brevi ovata, spiculis 3-4, suprema suepe sterili, valvis calycinis scabris mullistriatis $3-4$ aristatis, aristis patulis spicam excedentibus: Gussove, Flora Sicula, Volumen primum). Pare che la descrizione fatta dal nostro egregio e benemerito Botanico si accorda coi miei sospetti e con quanto ne asseriscono i pastori relativamente al nocumento che le reste della pianta apportano ai cavalli.

Avena sterilis. Volgarmente chiamasi avenacchia.Presso i coutadiui questa parola siguifica avena grossolana. I Botanici hanno dato a questa specie annuale il nome di 
sterilis. Ora io domando: se è sterile, perchè si moltiplica per via di semi? O la parola sterilis deve alludersi alla pochezza delle semenze che la specie produce? 0 forse al poco suo valor nutritivo? $\mathbf{O}$ alla conformazione della pannocchia? $\mathbf{O}$ ad altra causa?

Crnonon dactrlon. Da per ogni dove dicesi gramigna. La vera gramigna de'Botanici è il Triticum repens che io ho raccolto sempre nei vigneti e nei terreni silicei vicino al mare.

Hordeum merinum. Questa graminacea è poco o niente appetita dal bestiame. Quand' è in fioritura neppure le capre l'addentano. Cosa singolare per siffatti animali che divorando tutto, lasciano da parte questa specie di pianta! Nasce copiosamente lungo le vie, e contra i muri, ove spesso abbondano $\mathrm{i}$ formicai. $\mathrm{E}$ siccome le formiche trasportano indistintamente sotterra qualunque seme, ed in particolare quelli delle graminacee, non escluse le semenze dell'Hordeum murinum, così il volgo lo chiama grano di formica.

KoELERIA VILI.OSi varietas CULMIS LONGE EXERTIS. L' ho raccolta più volte nei terreni silicei marittimi alle foci del fiume. $\mathrm{E}$ furse pianta naturale di quei siti? O vive colà come pianta peregrina, perchè trasportati ne vengono $\mathrm{i}$ semi da altri luoghi mediante le correnti dell' Ofanto?

Ligurus ovatus. Graminacea comune che vien riconosciuta con diversi nomi vernacoli, cioè mustacci di militare; coda di sorcio; coda di volpe. Il terzo di questi nomi coincide bene col fatto. Guardate la spiga del Lagurus ovatus, e vi scorgerete grande somiglianza colla coda di volpe. 
LAPpigo RACEMOSA. Questa graminacea non si allontana da' luoghi marittimi e dai siti acquosi lungo i fiumi. Cresce vicino l'Ofanto a Barletta e a Canosa; nel 1837 la raccolsi a Manfredonia, e nel 1850 a Giovinazzo nei crepacci de' macigni calcari sulle coste del mare. Ivi il signor D. Vitantonio Cappelli nello stesso anno 1850 ne raccolse diversi saggi di una vegetazione rigogliosa, che era meraviglia il vederli cosi floridi crescere tra nudi sassi.

LoLuum мaxıмum. Chiamasi comunemente sciuoglio. Sarà forse la parola loglio guasta e corrotta in bocca ai contadini? Nel mese di aprile e maggio si ripuliscono i seminati di grano da tutte le erbe, massime dal loglio, il quale si vende fresco per ottimo foraggio agli animali. Qui permettetemi una piccola osservazione agraria economica a proposito del Lolium. Voi, per esempio, andate in Puglia, e ove più ove meno lo vedete vendere qual foraggio agli animali in Primavera unitamente ad altre erbe graminacee e leguminose, tutte spontanee che la Natura ivi produce abbondantemente. I Pugliesi dunque profittano di tanto bene, e non si curano di moltiplicare i prati artificiali. Al contrario, andate in un altro paese, e trovate piante diverse da foraggio, siano spontanee, siano coltivate; e tra queste ultime il granone, il panico, il trifoglio pesarone o incarnato, il lupino, la rapa ec. e ciò in Terra di Lavoro, ne' due Principati, e in altre Provincie. Queste usanze sono inveterate da lunghi anni, e sono figlie delle stesse circostanze locali e delle novelle coltivazioni, che (fra tante altre, le quali non si diffusero ) poterono piu estesamente prender piede in un 
paese. Cercate mo dopo questi falti d'introdurre cose nuove in mezzo a pratiche inveterate, e voi vi affaticherete invano con poco o niente profitto, se non date luogo all'opera di lunghissimo tempo che non sempre è dato vedere ad una medesima generazione che fu la prima a dare l'iniziativa alle novilà agronomiche. L' ignàme della China (Dioscoraea batatas), il Convolvulus batatas o patata dolce, forse vedranno diffondere le rispettive coltivazioni nel futuro $20 .^{\circ}$ secolo in Calabria e nella Sicilia. La Medicago falcata è otlima pianta leguminosa da foraggio, poichè cresce bene nella slate senza bisogno di acqua, ed è spontanea negli Abbruzzi, nelle Puglie, e altrove. (Vedete quanto ne dissi più innanzi). Ebbene, i Pugliesi sempre gridano che essi hanno bisogno delle praterie nei tempi estivi che non esigessero acqua : voi indicate la Medicago falcata ch' essi stessi calpestano; e intanto non trovate anima vivente che s'incaricasse a raccoglierne i semi e moltiplicarla !!!... Voi predicate ? ed è lo stesso che gridare alla luna! E quando nascerà qualche eroe agronomico nei futuri secoli, allora forse i posteri vedranno le praterie di siffatta leguminosa! Tauta è la possanza delle abitudini inveterate nell' economia campestre !!. Studiate un poco l'opera classica del Conte DE GaSpanin su i diversi limiti delle regioni agrarie, e vi convincerete del principio filosofico di questa verità di fatto. Intanto riprendendo il nostro principale argomento, aggiungiamo che l' essenziale sareble di fare lo studio comparato tra i valori nutritivi delle diverse specie spontanee che offre un paese. A cagion d'esempio, se in Puglia si studiasse sul valor nu- 
tritivo tra le specie di Lolium ed il Triticum repens, sarebbe una cosa utilissima pei proprietarii di animali. Ma come ciò fare senza lo studio della chimica e della veterinaria? Come giungere a tanta mèta senza amore e trasporto alle nobili occupazioni? Se i giovani chimici seguissero l'esempio del celebre MaLaGuts, la società guadagnereble il cento per uno!!

Lolium nultiflonum. Quest'altra specie addimandasi cigliata, e serve altresi a nutrire gli animali. Sembra che l'origine di questa parola provenga da ciò, che le glume delle piccole spighe essendo piuttosto compresse, somigliano un tantino alle ciglia degli occhi ; o se tale similitudine strana sembrasse, non si potrebbe la parola cigliata alludere alla facilita di cestire della pianta? $\mathbf{E d}$ in vero i campagnuoli ed il basso popolo dicono la pianta caccia $i$ cigli allorchè vogliono indicare che cestisce assai: ed il Lolium è precisamente in questo caso.

Arundo donax. Questa graminacea, detla canna comune o cannedda, giunge, nelle vicinanze dell' Ofanto, a produrre culni di una lunghezza di trenta palmi, giusta le asserzioni del Sig. D. Giuseppe Italia, il quale mi assicurava che a tagliare siffatti culmi cosi lunghi e grossi, è necessario adoperare l'accetta a replicati e sostenuti colpi.

Puragmites comunis. Non avendo questa pianta il culmo voluminoso come quello dell'Arundo donax, cosi i campagnuoli la chiamano cannazza, ossia canna di poco o nessun valore. Questa graminacea è abbondantissima lungo le sponde dell' Ofanto; e nella contrada della fumara al- 
lunga i suoi fusti o culmi, che dir si vogliano, rasente terra sino a quaranta palmi misurati da me espressamente nel mese di luglio 1847. Ne conservo un saggio nel mio erbario, il quale saggio è attorcigliato intorno a se stesso e ligato con un laccetto perchè potesse contenersi in un foglio di carta.

Secale cereale. Nasce nei seminati di grano. È cosa facile che le sue piante sortano da semi di segala frammisti a quelli del frumento prima della seminagione. Qui cade acconcio di ricordare le stesse osservazioni di Geografia botanica, secondo De Candolle, che feci a proposito della Vitis vinifera sylvestris e dell' Apium graveolens.

Stipa Pennata. Quantunque conosciutissima dai Botanici questa graminacea, ciò nondimeno mi gode l' animo ricordare agli amatori di piante la bellezza delle sue resle, le quali sono delicate oltremodo a foggoia di piume, e che, se fossero bene apparecchiate, potrebbero forse per le donne servire di ornamento ai loro cappelli. Ecco la descrizione che nella sua Flora Sicula ne fa il sig. Gussone, Volume primo. Panicula basi inclusa, valvis calycinis attenuato-acuminatis, aristis longissimis plumosis basi nudis, foliis rigidis glaucis canaliculato-setaceis, radice perenni.

Juxcus ACutus. Questa pianta palustre serve agli ortolani e ai vignajuoli. Ai primi per affasciare ortaggi, ai secondi per ligare i pampani.

Alludm pallens. Abila nei vigneti a terreno calcareargilloso nella contrada di Trojaniello.

Asphodelus luteus. I pastori e gli abitanti delle mas- 
serie ne allessano le tenere messe $e$ se le mangiano. Nasce sulle praterie, e vien addimandato avrusceno (pronunziate l'e muta).

Asphodecus ramoses. Cresce su i prati, di cui forma l'ornamento in primavera coi suoi vaghi fiori bianchi tendenti leggermente al roseo. I fusti disseccati si adoperano per combustibile dai pastori e contadini poveri. Nelle Reali Saline di Barletta i ragazzi ne costruiscono sediucce ed altri piccoli mobili per trastullo: indi li tuffano nelle vasche ove si fa il sale (previo il debito permesso delle Autoriti), e dopo qualche tempo ne li estraggono tutti coperti di sale puro e finissimo. Questa è quella pianta di cui si è tentata l' industria per la estrazione della sostanza alcoolica a cagione della crisi vinifera. E copiosissima in tutte le tre Puglie.

Mluscari comosem. I bulbi di questo giacinto o muscari si estraggono dai campi in gennajo ed in febbraio. Si nettano, si lavano, si spaccano in quattro parti, si allessano, e si mangiano conditi con olio e aceto. Altri li friggono, sia colla farina, sia colle uova, dopo di averli allessati: e v' ha di quelli che senza pulirli, li sottopongono alla bragia infocata, e dopo li nettano, e se li mangiano così arrustiti. Questa è l'usanza de'Pugliesi. I bulbi hanno un colore rossastro tendente al roseo, e sono cosi nauseosi ed amari, da non potersi digerire che dal solo slomaco di bronzo : ed io fin dalla mia fanciullezza ebbi un' avversione pronunziata contro siffatto cibo grossolano distinto coll'epiteto di lambascione. Del resto; de gustibus non est disputandum. Dicesi che ve ne sono due varietà quella ros- 
sastra, e quella bianca. Di tutte due la seconda è perniciosa, perchè indigeribile e produltrice di forti dolori di testa e scioglimento ventrale. Io mi ricordo sin dalla tenera età i lambascioni bianchi e quelli rossi ; ma nel tempo della mia carriera botanica non ho avuto la fortuna di distinguere queste particolarità di piante. Sarà forse la bianca una varietà distinta del Muscari comosum? Sarà una specie di Scilla? Sarà in fine qualche altra specie di Ilyacinthus? Col tempo i Botanici verificheranno questo fatto naturale.

ORNITHOgalum VILLOSUM var. FloRiBUS ODORIS.

Gagéa villosa varictas flomibus odoris. mese di marzo 1841 sulle praterie al Sud della Città, e propriamente dirimpetto la masseria Centrone e Passero sul tratturo insieme col signor D. Luigi Passero (che sempre gentilmente si è meco cooperato alla raccolta delle piante, tutte le volte ch' eravamo in compagnia) raccolsi, taluni saggi di siffatta gigliacea, i quali, disseccati, furono da me inviati in Napoli al sig. Gussone. Egli colla sua solita cortesia e bontà mi scrisse una lettera, dicendomi che quelle piante erano varietà dell' Ornithogalum villosum ossia arvense. Poi mi soggiungeva che almeno cosi dovevano ritenersi, sendochè le piante grasse, quelle bulbose, e diverse altre vogliono essere studiate allo stato fresco, poichè disseccandosi perdono taluni caratteri in modo che si rendono difficili a riconoscersi bene. Nel 1846 facendo istanza presso il prelodato Botanico in Napoli perchè si degnasse rivedere nel suo pregiatissimo erbario i miei saggi inviatigli da Barletta, lo stesso pieno di amabilita sod- 
disfece le mie premure, ed un giorno mi disse : Ho esaminato il vostro Ornithogalum, e veramente merita l' attenzione dé Botanici per definirsi, o pur no, quale specie nuova. Siffatta varielà era stata paragonata da me coi saggi del vero Ornithogalum villosum (raccolto nello stesso marzo 1841 nei seminati di grano), ed aveva queste particolarità , che le sue foglie erano più corte e più larghette di quell' altra comune nei campi ; alquanto ciliate ai loro margini ; con scapo più corto; portante fiori meno numerosi, da due a tre ; corolla non lineare, ma poco più larga e quasi ottusa all'estremità delle lacinie, e di un odore gratissimo. Tutto il contrario si osservava nei saggi della specie comune, i quali ultimi presentati al sig. Gussone, egli li riteneva per tali, e non per varietà della specie, come lo erano quelli a corolla odorosa.

Scilla manitima. Per la feracità del terreno lungo le rive dell' Ofanto lo scapo raggiunge l' altezza di sei palmi misurati da me soventi volte. Coperto di fiori bianchi per quasi tutta la sua lunghezza, fa un bel vedere nel mese di agosto e settembre in mezzo alle praterie ed ai cespugli. Il suo aspetto e portamento dà un poco del romantico in quei luoghi alquanto solitarii e del tutto silvestri. Cresce su i pascoli aprici del tratturo e nei bassi fondi vicino al fiume, come poc' anzi ho accennato. Havvi la sola varietà a cipolla rossa; quella a bulbo bianco non l' ho vista mai. In Napoli si è introdolta ad uso di ornamento nelle sale per la vaghezza de' fiori che si schiudono senza che la pianta stia nel terreno, venendo le rispeltive cipolle dalla cosliera di Sorrento. Il succo della Scilla mariti- 
ma mescolato al formaggio e al vetro polverizzato si adopera dai Pugliesi per uccidere i topi. Taluni vantano a cielo l' efficacia di questo rimedio, altri poco o niente. Checchè ne sia, certo si è che i sorci e le cosi delte zoccole saranno sempre il tormento delle famiglie, dei bastimenti, delle campagne coltivate, e de' giardini privati : ed il problema della loro disfatta sarà sempremai d'impossibile soluzione.

Scilla autumalis. Abita nelle vicinanze del fiume nei fondi a base silicea.

Scilla undolata? Una pianta di Scilla, senza fiori, fu raccolta da me nelle vicinanze dell'Ofanto in Autunno (non ricordo di quale anno), e presentata in Napoli al sig. Gussone. Egli mi disse aver qualche sospetto per la Scilla undulata: ma poi soggiunse, mancano $i$ fori, $e$ mi sembra diffeile che possa vegetare sulle sponde dell' Ofanto. In questo anno 1857 ho consultata l'opinione del chiarissimo Botanico sig. Gasparrini ; e questi era di avviso che la si fosse cosa piuttosto facile a rinvenirla in quei luoghi. Io ho voluto intanto citare questo fatto, chi sa col tempo se qualche amatore di Botanica potesse rintracciarla. Negli studii delle Scienze naturali senza costanti ricerche e senza una rigorosa osservazione, non si possono assodare certi punti scientifici i più difficili, che sono ancora in via di problemi. V' ha dei fatti disparati in Natura che sembrano diametralmente opposti alle leggi teoriche stabilite da cinquant' anni fa ; e intanto i Naturalisti a misura che studiano, si avveggono che i medesimi sono collegati coll' ordine del tutto, quantunque non ancora 
siansi spiegate le rispetlive cause, dalle quali emanano. Il sig. De Cannolle parlando di quelle specie di piante i di cui individui si trovano divisi tra due o più paesi separati a grandi distanze, sviluppa le più sublimi idee scientifiche e filosofiche intorno a siffatto argomento. Egli chiama giustamente queste piante espèces disjointes, e dice così: “ L'épithète de disjointe me paraît convenir à certaines a espèces dont les individus se trouvent divisés entre deux “ ou plusieurs pays séparés, et qui cependant ne peuvent “ être envisagées comme ayant été transportées de l’ un à " l' autre, à cause de quelque circoslance tenant, ou à la “ structure des graines, ou à la manière de vivre des “ plantes, ou à l’ éloignement considérable des pays d'ha" bitation. Comment des espèces peuvent-elles se trouver " ainsi séparées, sans transport probable, ni même pos" sible? Ce doit être par l'effet de causes antérieures à " l'ordre de choses actuel. Voilà ce qui me fait attacher " une grande importance à de pareils faits ». E più appresso, continuando a ragionare da gran Filosofo, si esprime in questi termini : "On ne peut se défendre aussi de " remonter à la distribution primitive des espèces, comme ( ayant produit peut-être ce singulier mode d'habitation ». A questo proposito io domando ai Botanici : la Plantago major è piuttosto pianta dei luoghi bassi e palustri, e come va che il sig. Cavaliere Michele Tenone la trovò sulla cima di monte Corno negli Abbruzzi, che secondo taluni Fisici osservatori sarebbe elevato dal livello del mare per 1588 tese ? Sopra una scala più ristretta rispetto alle lontananze, non sarebbe questo un esempio analogo alle plan- 
qes disjoinles del sig. De Candolle? I signori Giuseppe Antonio Pasquale e Giulio Avellino riferiscono nella loro Flora medica della Provincia di Napoli, stampata nel 1841, che essi la raccolsero sul picco della montagna di San Michele sopra Castellammare. Non gli è questo un altro esempio in piccolo ? Mi si dirà ; ma nel caso della Scillce la somma delle temperature, cioè quella che risulta dall'accumulazione delle temperature utili alla specie (DE Candolle; Geografia bolanica; volume primo), non si trova forse in Puglia, e quindi vane riuscirebbero le ricerche. Io rispondo che non sarebbe tanto lontano dalla naturale distribuzione geografica il rinvenire siflatta specie di Scilla nella Puglia. V' ha tauti e tanti esempii simili in molte Flore delle varie regioni del globo, che non si è fatto altro dai Botanici, che elevarsi di molto per cominciare lo studio sulla traccia delle vere cause di simiglianti fatti . “ L'étude des limites d' espèces sera toujours très-com"pliquée ». (Dice De Candolie, parlando de' limiti polari delle specie spontanee, in pianura). "Voici pour" quoi. Une espèce peut être arrêtée dans un pays par le " froid de l'hiver; plus loin, du côté ouest par exemple, "le froid sera rare et moins intense, mais il ne fera pas " assez chaud pour avancer la floraison, ou bien l'été ne " sera pas assez favorable pour que les graines puissent “ mûrir en automne, ou enfiu l’ automne sera trop froid. "Ainsi on conçoil qu’ une mème espèce puisse ètre limi" tée dans un point par une cause, dans une autre dire" ction par une seconde cause, plus loin par une troisic« me, au de là par une quatrième, ect. Ceci est d'autant 
" plus vrai que l'humidité ou la sécheresse, réparties di“ versement dans les mois de l’année, peuvent agir aussi " comme causes de délimitation, surtout dans les pays " méridionaux (avete capito, Lettore?), et, se mélan“ ger avec les effets de la température. De là des phéno" mènes bizarres dans les limites d'espèce, el une foule " d'exemples, qui paraissent des anomalies, et qui cepen" dant pourront peut-être s'expliquer, si l'on scrute " chaque fait at si l' on compare attentivement les limites " bien constantées d'une espèce avec les conditions de “ température el d'humidité, dans toute l'étendue de ces " limites ». (Qui permettetemi per digressione farvi riflettere, che il sig. DE Daum ha pubblicato a Stellin, in Germania, nel corrente anno 1857, una dotta Memorietta: De l'infuence du climal sur les époques de la végétation, ove si trovano principii analoghi al nostro principale argomento ). Mi si obbietterà, che il signor DE Candote parla sopra una vasta scala di uma o più grandi regioui della superficie terrestre, e che il punto dell' Ofanto essendo una ristrettissima località, non può meritare l'attenzione di si alto grado. Alla qual cosa rispondo che, o la scala è vasta, o è ristretta, il principio filosofico in rapporto all' umidità e alla temperatura, confacenti alle diverse specie, è sempre lo stesso, e da potersi applicare ad ogni punto del globo, tutte le volte che le condizioni fisiche sono in armonia colla natura delle piante e colle esigenze della loro naturale distribuzione geografica. Il forte a sapersi, e che io domando ardentemente, si è quanto segue : Ci è stato alcuno che si diè la pena di os- 
servare e calcolare esattamente la massima e la minima di temperatura e di umidità lungo le rive dell' Ofanto per tutto il corso dell' anno? Si sono fatte ivi le osservazioni sugli effetti della luce indiretta all'ombra degli alberi e dei cespugli, e su quelli de' raggi diretti del sole, per una medesima specie di vegetabile erbaceo? Si sono messe a calcolo le influenze de' raggi chimici della luce ? Questi sono studii fisici bastantemente delicati e sublimi perchè vi si occupi solamente l'uomo della Scienza, ornato di flemma e di costanza per un periodo, non interrotto, almeno di un anno intero! Donando io di più : Ci è stata persona che siasi impegnata di frugare, in quegli erbaggi spinosi ed intricati, per una serie, non interrotta, per lo meno di quattro stagioni autunnali? Io, con tutto che ho frequentati quei luoghi le cento volte in tutte le quattro stagioni, ed in ispecialità dalla Primavera all' Autunno, pure a grandi stenti raccolsi la sola pianta di Scilla senza fiori; e Dio sa come ne riportai le mani e gli abiti laceri per uscire da quel labirinto di spine orrende e di macchieti annosi, accavallati, per cosi dire, gli uni a fianco degli altri, e che il solo fuoco potrebbe decimare. Finalmente domando: Ed ancorchè da' Fisici e dai Botanici si trovasse vicino l'Ofanto la temperatura ed umidità favorevole alla natura della Scilla undulata ( pianta dell'Africa settentrionale; vedete a pagina 142 ciò che sta accennato e promesso in riguardo della Scilla undulata, allorchè parlai della Silene rubella), e che ivi se ne riconoscesse la naturale e geografica abitazione ; 0 in altri termini, che il limite della sua abitazione si distendesse un poco più al Nord e all'Ovest del- 
l' Ofanto, verso la Provincia di Foggia ; chi ci assicura che le correnti del fiume non ne distruggessero qualche raro saggio, sia trasportandone i bulbi al mare, o seppellendoli nella melma, per lungo tempo, ove potrebbero facilmente marcire? Se le correnti impetuose dell' Ofanto svelgono gli alberi di olmi annosi, s' immagini cosa possano fare di una piccola pianta erbacea. In breve conchiudo, che v' ha bisogno di altre premurose ed esatte ricerche della pianta in parola: che il non trovarla mai, non potrebbe questo fatto negativo decidere a priori sulla impossibilità della sua abitazione in quella contrada, senza gli antecedenti studii di temperatura e di umidità del luogo in rapporto ai naturali bisogni della specie: e che in fine l' esplorare assai minutamente gli erbaggi dell' Ofanto pieni di spine impenetrabili, non è opera di un sol giorno o di più passeggiate, nè del solo amatore, senza la guida e l' ajuto di persone pratiche del luogo che vedessero e raccogliessero tutto. Io non dimenticherò mai $i$ saggi consigli ricevuti dai signori Tenore e Gussone, i quali, quando mi dettavano lezioni, più volte mi hanno delto cosi : "Nella ricerca delle piante non vi stancate mai : non vi " limitate solamente alla loro raccolta in una sola volta: " ripetete le peregrinazioni in diverso tempo e in diversi "luoghi per le stesse specie; studiate tutto che loro è " d' intorno: paragonate le diverse cause e i corrispon" denti effetti: studiate il terreno nelle sue qualita, la "posizione topografica, e tutte le altre circostanze fisiche "le quali potranno accompagnare o influire alla esistenza " delle specie : fate un esalto studio comparato fra i mol- 
" tiplici saggi. A questo modo col tempo vi convincerete " che non tulti i luoghi esplorati sono stati bene esplo" rati! " Ed in verita questi principii si sono sempre verificati colla scoverta di novelle specie di piante che tutto giurno si vanno pubblicando nelle diverse Flore che i Botanici descrivono nei varii paesi dell' antico e del nuovo Continente.

Tulipa sylvestris. I contadini contradistinguono questa specie di tulipano colle voci fore di lambascione. E cosa originalissima siffatta denominazione. I campagnuoli nei mesi di gennajo e di febbraro sanno riconoscere e distinguere benissino i bulbi del Muscari comosum (che è il vero lambascione de' Pugliesi, siccome dissi più avanti); ed intanto in primavera credono che i fiori gialli della Tulipa sylvestris siano prodotti dai lambascioni, i quali appartenendo al vero Muscari comosum, portano fiori di un colore tetroceruleo. Eccone la descrizione fatta dal sig. Gussone nella sua Flora Sicula, Volume primo: Corollae fertiles juniores atrocoeruleae, sub anthesi sordide virentes; denticulis albidis, obtusis, recurvis; steriles laete cyaneae. Racemo elongato, corollis cylindraceis horizontalibus subapice angulatis fauce subclausis, summis sterilibus longissime pedicellatis coloratis erectis. Non crediate, o Lettore, ch' io esiger volessi da' contadini cognizioni botaniche. Sarebbe puerilità il pensarlo. Dico solamente esser cosa originale che mentre i medesimi distinguono bere nella stagione d'inverno i bulbi del vero Mluscari comosum, pensano poi nella primavera che gialli siano i suoi fiori. Fiori i quali sono odorosi, ed appartengono al tulipano silvestre e non già al così detto lambascione. 
Tulipa apula. I fiori di questa specie, descrilta dal signor Gussone, sono rossi, e si raccolgono da'campagnuoli per ornare gli Altari nelle Chiese al tempo della settimana Santa: ecco perchè si addimandano volgarmente fiori $d i$ monumento.

Lemna gibba. Tutte le specie di Lemna vengono riconosciute colle parole màmma dell' acqua. Tutti sanno che nei giardini abbandonati e negli orti le vasche di acqua spesso si coprono di un manto di verdura, formato ordinariamente da più specie di Lemna; e questo fatto naturale più frequentemente si osserva nei canali de' luoghi palustri. Per lo che le acque non avendo corso, e poco o niente contatto coll' aria atmosferica, facilmente si alterano: e se vi si aggiungono le miriadi d'insetti che sogliono annidare e morire tra le piante di Lemna, sarà cosa agevole il riconoscere le principali cagioni per cui quelle acque di leggieri si corrompono, tramandando quel fetore intollerabile tanto nocivo alla salute pubblica e cosi ben conosciuto dall' universale degli uomini che in vita loro avvicinarono siffatti luoghi. Gli ortolani di Barletta si esprimono intorno alle specie di Lemna, servendosi delle voci màmma dell' acqua, quasi alludere volessero all' idea che le Lemne cuoprono e difendono le acque allo stesso modo che fanno le galline vascondendo i loro piccini sotto le ali, o come le madri che stringono fra le braccia e avvolgono di un mantello i proprii figliuoli. Se non che vuolsi avvertire che nelle conseguenze di questi falti si veggono cose diametralmente opposte; poichè le galline e le madri cercano difendere e cautelare la loro prole, mentre poi le 
Leme per una legge tutta noturale tendono alla corruzione delle acque, allo stesso modo che il Viscum album crescendo sul mandorlo e sul sorbo, ne accelera il deperimento e fors' anco la morte. Veggasi quanto ne ho detto circa il Tiscum album, il quale vien riconosciuto colle voci vernacole màmma dell' albero.

Himanthoglosscm mincinum. Siffatta orchidèa fu da me raccolta per la prima volta in maggio 1847 negli erbaggi e fruticeti lungo il fume Ofanto, e precisamente nelle vicinanze de' colli di Canne in quelle praterie e macchieti denominati comunemente mezzàne del medico: mezzàne del Canonico Siero. I massari di Barletta si servono della parola mezzàna per esprimere ed indicare una estensione di terreno, destinato al pascolo degli animali. Io non ho potuto mai capire il vero significato allusivo di questa parola vernacola mezzàna. Sarebbe curioso rintracciarne l'origine corrispondente al vero significato. Debbo qui intanto per sentimento di gratitudine manifestare che nella raccolta di questa bella specie d'Himanthoglossum hircinum molto si cooperarono i Fratelli Russo, di Barletta, sendochè ad estrarla da un terreno estremamente argilloso (a molta profondità) e coperto di orribili spine de' frutici e cespugli, si durò assai fatica e stenti. I sudetti Fratelli Russo in moltissime occasioni mi hanno per loro bonta portate varie piante che trovavano in diversi luoghi delle campagne di Barletta, e che io slesso poi soleva raceogliere di bel nuovo in altri siti diversi. Valgano dunque 'jueste parole per attestare la verità de' fatti, e per provare che il periodo di venti anni (dal 1837 al 1857) nou 
potè cancellare in the la dolce memoria delle mie ristrette, ma ripetute peregrinazioni botaniche, per talune delle quali i Fratelli Russo mi spiegavano tutto l' impegno di una sincera amicizia a favorirmi.

OpIrRys lutea. Nasce nei siti ombrosi a bacio in terreni marnosi calcari, dirimpetto il distrutto casino di Fraggianni (nobile ed antica famiglia barlettana) e quello di Santo Luca, e vegeta del pari sulle praterie ombrose non molto lungi dalla masseria Centrone, e precisamente ove si cavano i tufi per la costruzione delle case.

Opurys aracinites. Abila nei terreni marnosi argillosi unidi esposti a bacio dirimpelto il mare all' Ovest della Città, e propriamente mezzo miglio distante dal casino del fu Marchese Affaitati.

Orchis moravica et Orcins militaris. Queste due specie crescono nei fruticeli lungo il fiume, ed in ispecialità negli erbaggi denominati Colucci; Spinalba; Chiuppo, e luoghi simili.

Sparganium ramosom. Abita alle paludi, lungo i canali che conducono l' acqua al mare, comunemente detti cunnutti, corruzione di condotti o aquidotti. E una bella specie palustre, e non si trova che alla distanza di quattro in ciuque miglia all' Est della Città.

Typia mina. Cresce ae bassi fondi pantanosi alla contrada di casavecchia verso la via di Canosa. II Dottor Vincenzo Casale, mio defunto Zio, mi diceva di averla ivi spesse volte raccolta. Anch' io nell' agosto 1844 in compagnia di mio defunto Padre e di mio Fratello la trovai colà in vegetazione avanzata. Questa specie è meno comune della Typha latifolia. 
Polyponus ovatus el Polyporus variegatus. Nel 1844 e 1847 a mie istanze il sig. D. Ferdinando Cafiero fece raccogliere sugli alberi di pero selvaggio (Pyrus cuncifolia) queste due specie di funghi, e mi usò la gentilezza d' inviarmeli a Napoli. lo li presentai all' egregio e benemerito Professore sig. D. Francesco Brignnti, il quale mi favori indicarmene i nomi, essendo egli versatissimo in questo ramo di Storia naturale. - Oltre il fungo comune mangereccio che nasce a piè della Ferula communis e altrove, vi sono altri funghi, che più volte ho visto, ma non ho raccolti per la difficoltà di potersi disseccare. Fra questi me ne ricordo uno tutto tulto di colore giallo carico sopra un tronco annoso di olivo nel giardino della masseria di Santo Luca. Ma io allora non aveva che dodici anni di età (nel 1829), e non avendo cognizioni di Botanica, non ebbi premura a farlo distaccare dall' olivo con un pezzo di scorza dello stesso albero, e cosi si sarebbe potuto inviare in Napoli ben condizionato. Feci ricerche nello stesso luogo negli anni 1844 e 1847 per vederlo riprodotto, ma infruttuose mi riuscirono.

Lichen.....? Sulle tufare, al petraro e luoghi simili; come anche sugli alberi annosi di pero selvaggio, di carrubbe, di olivo, di fico, e di mandorlo vegetano varie specie di licheni, che sempre ho visti, e mai raccolti.

Hypnum. . . . ? Lo raccolsi vicino alle vasche della fonIana di Canne. Molte specie di muschi crescono su pei muri, sulle parieti, ed in tutli i luoghi ombrosi ed umidi; non escluse le anfrattuosità de' macigni calcari e de' massi tufacei tagliati da anni ed anni, le quali conservano quella 
temperatura ed umidità tanto necessarie allo sviluppo delle critlugame : e negli erbaggi dell' Ofanto anche se ne trovano in grande copia.

Lycoperdon pedunculatum. Fu da me raccolto in autunno 1847 nei terreni silicei lungo le rive dell'Ofanto $(a)$.

(a) L'Ofanto è piuttosto un torrente, anzichè un fiume; e se io gli ho dato questo nome, ciò è stato per tre ragioni :

$1 .^{a}$ la storia riferisce che negli antichissimi tempi era navigabile ; e presentemente dal mare sino al ponte crollato ( tratto di circa due miglia ) si possono remare grossi palischermi :

2. ${ }^{a}$ è rinomato per la famosa battaglia di Canne, e pare giusto che con ciò si avesse un certo riguardo:

$3 .^{a}$ la rigogliosa vegetazione erbacea ed arborea si distende per lunghissimi tratti alle sue sponde, la qual cosa è una pruova evidentissima delle sue acque copiose, le quali se fossero bene incanalate, offrirebbero una profonditả perenne e regolare. E se nei mesi estivi si passa a guado e a piedi asciutti in molti suoi punti, ciỏ non dipende dalla scarsezza delle acque, ben vero da altra causa tutta naturale, ed eccola:

Il suo letto è assai ampio, ma non ricordo la precisa larghezza. Durante la stagione d' inverno le strabocchevoli correnti depongono in varie parti una quantitì sterminata di ciottoli di peso diverso, da un' oncia sino a più rotola, che sono materiali degli Appennini. Ora siffatti banchi di ciottoli formano tante prominenze nel centro dell'alveo, e conseguentemente nella stagione estiva impediscono il libero passaggio delle acque modeste si, ma perenni. Queste dal canto loro divergono e vanno a depositarsi nei bassi fondi adjicenti. Ivi ristagnano, si corrompono, ed esalano continuamente nei caldi mesi, d'onde ne viene l' aria malsana, la quale è tanto più molesta e nociva per quanto maggiore è la quantita delle sostamze organiche animali e vegetali che si guastano e putrefanno nell' acqua. In siffalti laghetti avete la profondità di otto a trenta palni e piu con un 
fondo tutto melmoso, nel quale chi pone piede, vi è attratto come da una specie di forza magnetica : e piì lo sventurato si dimena per uscirne, più si affonda la sua salma, finchè trova irreparabilmente la morte !!...

Che se poi a questi fatti naturali aggiungete una causa tutta artificiale, quella cioè del canale di comunicazione tra l' Ofanto c il lago di Salpi, vi convincerete maggiormente che le sue acque restano più scarse, non per difetto positivo in quantità, ma per decimazione prodotta dalla mano dell' nomo, non esclusa la trascuraggine di rispettare gli argini e di consolidarli con numerose piantagioni di alberi. E da ultimo se ponete mente all' ingordigia di dissodare gli erbaggi, poco lungi dalle acque, per seminare grano, avrete continuamente sott' occhio due grandi mali : 1. ' l'agevolazione alle acque nel dissiparsi in più ampio spazio e quindi la rispettiva diminuzione nel centro del loro letto: 2." l' allagamento e la rovina nei campi vicini, che fa spavento nel vederli !!! E dunque massimo errore di economia sociale quello di dissodare i fondi erbosi lungo i fumi! Mai dissodamento ci dovrebb' essere in siffatti erbaggi ; che anzi l' obbligo correrebbe di piantare alberi, e poi alberi : su di che sempre si scrisse e mai si esegui !

Qui cade in acconcio richiamare la nostra attenzione sopra un punto di vera economia agraria: ed eccolo. Noi riconoscemmo in sul principio Barletta situata in un fossato. Visitammo all' Est della Città estesissimi campi palustri: presentemente ritocchiamo ciò che vedemmo più innanzi, cioè una vastità di luoghi pantanosi nelle vicinanze dell' Ofanto. Ciò premesso, chi non vede che Barletta sta in mezzo a due bacini di acqua, le di cui esalazioni fomentano le nebbie in certi mesi dell' anno? Se dunque ciỏ è vero, siccome gli stessi Barlettani lo attestano, perchẻ piantare olivi nei siti bassi? Gli è un errore ed un capitale male impiegato! Siffatte nebhie sono piuttosto insensibili e danneggiano gli olivi ; ma non raggiungono mai quella intensità di cui parlai a pagina 68 , per lo che mi protesto che non intendo con cio cadere in contradizione. 



\section{R I A S S U N T O}

Ora vorrete, cortese Lettore, riassumere quel tanto che vi ho detto ed esposto? Eccomi a roi.

Nella dichiarazione vi ho manifestato un cenno storico del mio progetto sul già eseguito lavoro, il quale fu da me concepito fin dal i 855 .

Con due lunghe passeggiate vi ho messo sott'occhio due quadri, de' quali uno vi espone la posizione topografica delle campagne di Barletta, e l'altro la natura dei loro terreni. Però debbo confessarvi francamente che dimenticai indicarvi le analisi de' terreni presso l'Olanto, eseguite da me nel i 847 e pubblicate a mo' di specchietti sul giornale il Propagatore delle Scienze Naturali: e però a volervi meglio servire, ve le presenterò in migliori occasioni. 
Una lunga serata di studio vi ha fornito delle notizie meteorologiche del mio paese, tratte dalle opere del benemerito Abate Giovene, da Molfetta, e comparate con quelle dell' illustre $\mathrm{DE}_{\mathrm{E}} \mathrm{G}_{\mathrm{A}}$ SPARIN ; ed avete visto come si accordano questi due grandi Uomini sul clima di Puglia : il primo pei fatti locali, ed il secondo pei fatti generali.

Durante il cammino di quattro giorni di ritorno alla Capitale noi abbiamo data un' occhiata all' elenco delle piante spontanee barulensi da me raccolte nelle varie occasioni : e perchè in questo tratto di tempo non ci fossimo annojati per la lotlura del medesimo, abbiamo creduta cosa utile tener conto de' pochi nomi vernacoli di piante, che in dieci anni ( dal x 837 al r 847 ) mi fu dato raccogliere con qualche stento presso le persone di campagna.

Inoltre solto forma di piccioli quadri sinottici

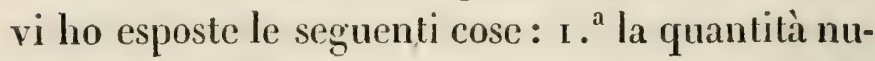
merica delle famiglie naturali, dei generi, delle specie, e delle varietic in rapporto alle diverse sezioni delle piante Dicotiledoni, Monocotiledoni, e Acotiledoni : 2. ${ }^{\text {a }}$ le somme generali delle famiglie, generi, specie, e varietà : 3. ${ }^{a}$ la proporzione maggiore delle Dicotiledoni sulle $\mathrm{Mo}$ nocotiledoni, ed abbiamo trovato precisamente 
le leggi di Geografia botanica stabilite da $\mathrm{DE}_{\mathrm{E}} \mathrm{C} \Lambda \mathrm{A}$. DoLLE ( figlio ) circa la distribuzione naturale dei vegetabili sulla superficie terrestre, per lo che ne citammo più innanzi le due leggi formolate dal prelodato Botanico Ginevrino : 4. ${ }^{a}$ il numero dei generi per ciascuna famiglia nelle Dicotiledoni, Monocotiledoni, e Acotiledoni, c la proporzione del numero delle famiglie di rincontro a quello de'generi.

Da ultimo con varie note ed osscrvazioni sopra diverse piante vi ho discusso qualche punto scientifico, e vi ho date svariate notizie sulle lo. ro località, sulle usanze rispettive de' contadini, sulle voci volgari, e tante altre cosette. Ed è così che vi ho presentato in breve un piccolo lavoro concepito sin dal i 835 , e scritto ed eseguito dal 27 febbraro sino alli ro di maggio corrente anno 1857 .

Ora spetta a me il dovere di ringraziarvi per la gentile compagnia tenutami durante le escursioni botanico-agrarie nel tenimento barulense. A voi il dritto di riposarvi e attendere da me ulteriori servigi con altri lavori che mi farò un pregio presentarvi a miglior tempo. State sano.

Napoli, li dieci di maggio 1857 . 



\section{N D I C E}

Delle cose Contenute Nel PREsente volume

Dichiarazione dell' Autore . . . . . . . pag. 3

I. Posizione topografica delle campagne di Barletta . 9

II. Rapido sguardo sulla natura de’ terreni. . . 32

III. Notizie sul clima pugliese, e precisamente su quello della Peucezia . . . . . . . . . . 43

Catalogo delle piante spontanee nell' agro barulense. . 77 S. Sezione 1. ${ }^{a}$ Piante Dicotiledoni . . . . . ivi

S. Sezione 2. ${ }^{a}$ Piante Monocotiledoni. . . . 109

S. Sezione 3. ${ }^{a}$ Piante Acotiledoni . . . . . . 116

Elenco de’ nomi volgari di talune piante. . . . . 118

Numero totale delle famiglie, generi, specic, e varictà. 123

Somme generali. . . . . . . . . . . . 124

Proporzione maggiore delle Dicotiledoni sulle Monocotiledoni . . . . . . . . . . . ivi

Numero de' generi per ciascuna famiglia nelle Dicoliledoni. . . . . . . . . . . . 126 
Propor sione del numero delle famiglie di rincontro a quello de’ generi nelle Dicotiledoni . . . pag. 129

Numero de' generi per ciascuna famiglia nelle Monocotiledoni . . . . . . . . . . . . 131

Proporzione del numero delle famiglie di rincontro a quello de’ generi nelle Monocotiledoni . . . . 132

Numero de' generi per ciascuna famiglia nelle Acotiledoni . . . . . . . . . . 133

Proporzione del numero delle famiglie di rincontro a quello de’ generi nelle Acotiledoni . . . . . 134

Note ed osservazioni intorno a varie piante . . . 137

Riassunto . . . . . . . . . . . . 207 
A pagina 16 verso 10 ugnuturi Leggete ngnuturi

" 26 verso 27 ed in altre e di altre frutta frutta

“ 44 verso 23 longitudine tale longitudine orientale

( 48 verso 21 adottato adattato

( 59 verso 16 della dalla

( 61 verso 10 adattato adottato

« 96 verso 12 Lotus augustissimus

Lotus angustissimus

« $\mathbf{1 3 5}$ verso 15 che le ha rilevate che lo ha rilevato 



\section{Pronto pel torelio}

\section{LE PALUDI DI NAPOLI}

DESCRITTE ED ILLUSTRATE SIN DAL 1846

Con un quadro sinottico a sette colonne

\section{In corso di compilazione \\ LEZIONI DI AGRICOLTURA}

A D USO

DE' PROPRIETARII DEL REGNO

\section{PREZZO DEL PRESENTE VOLUME}

$$
\begin{gathered}
\text { C } 0 \text { N F I G U R A } \\
\text { grana } 45
\end{gathered}
$$

Trovasi vendibile Vrco SAN Guduseppe Maggione

$$
\text { (ai Guantaj) N. }{ }^{\circ} 22
$$

Presso il Librajo Federico Stikler al largo del Castello e presso la Stamperia del Fibreno 


\title{
CONSIGLIO GENERALE
}

\author{
DI PUBBLICA ISTRUZIONE
}

Napoli 20 maggio $185 \%$.

Vista la domanda del Sig. Raffaele Marotta, il quale ba chiesto di porre a stampa l'opera del Sig. Achille Bruni intitolata - Descrizione Botanica della Campagna di Barletta.

Visto il parere del Regio Revisore Signor D. Pietro Calandrelli.

Si permette che la suindicata opera si stampi; però non si pubblichi senza un secondo permesso, che non si darà, se prima lo stesso Regio Revisore non avrà attestato di aver riconosciuto nel confronto, esser l'impressione uniforme all'originale approvato.

Il Consultore di Stato Presidente Provvisorio-Capomazza.

Il Segretario Generale - Giuseppe Pietrocula.

\section{COMMESSIONE ARCIVESCOVILE}

Nihil obstat

Vincentius Tenore

Censor Theologus
Imprimatur

Pel Deputato

Lepoldo Ruggiero

Segretario. 


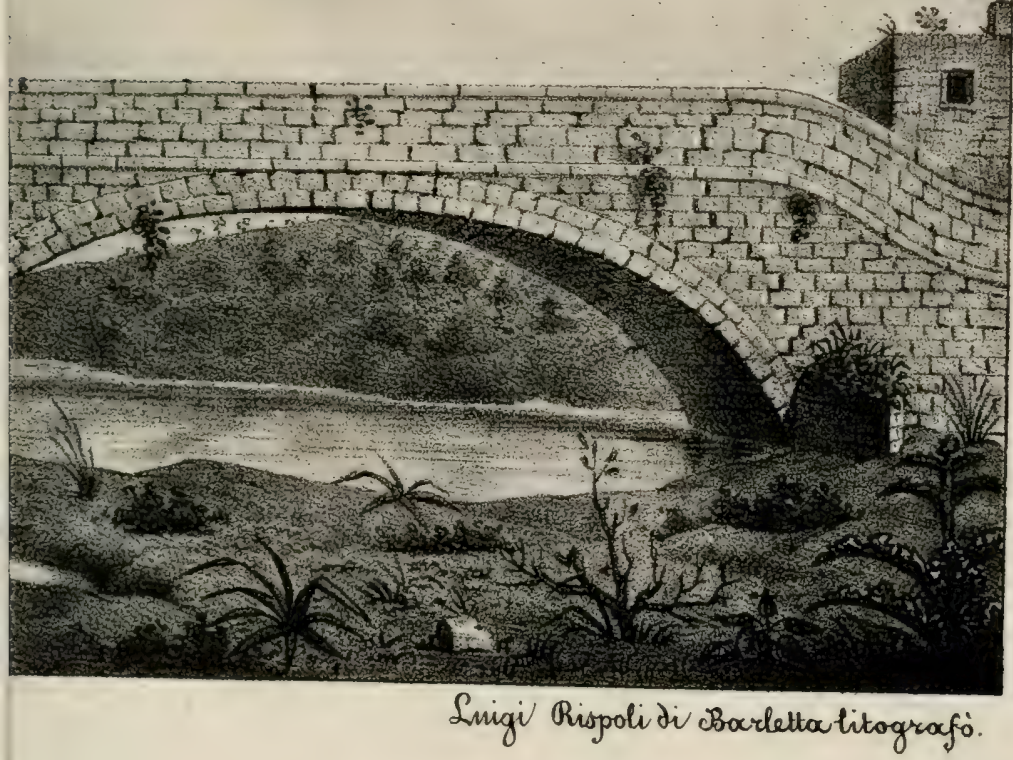




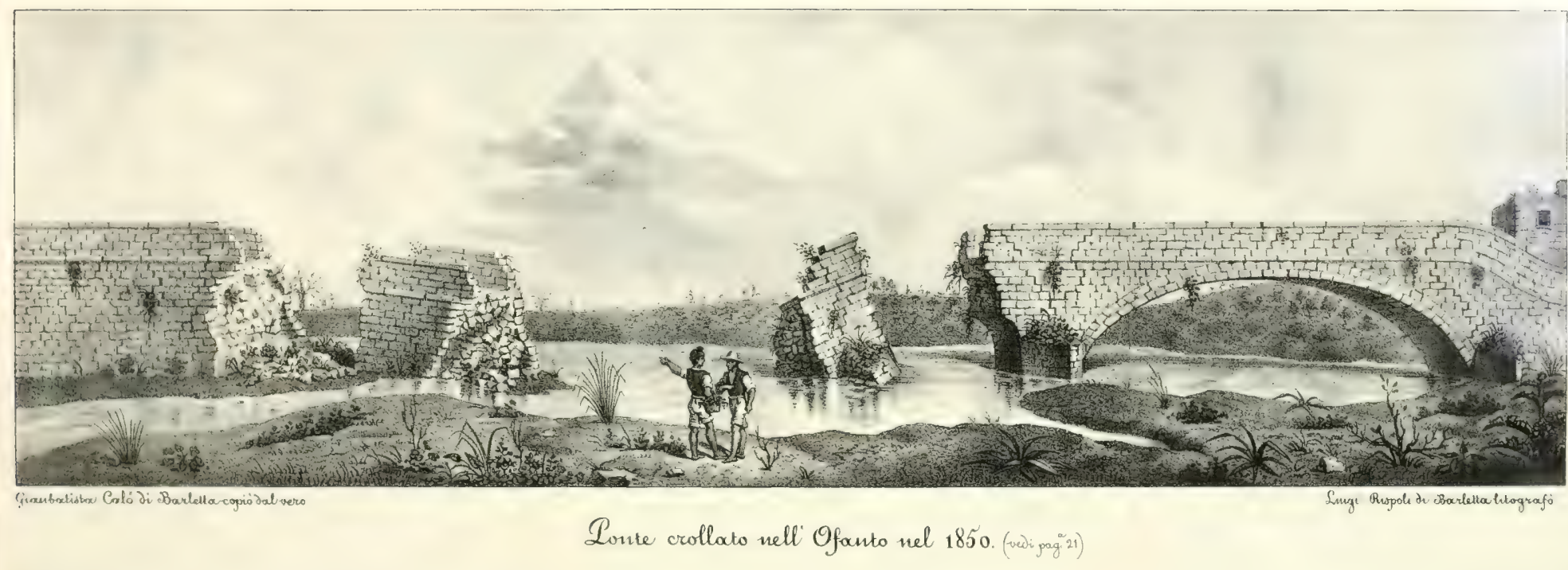





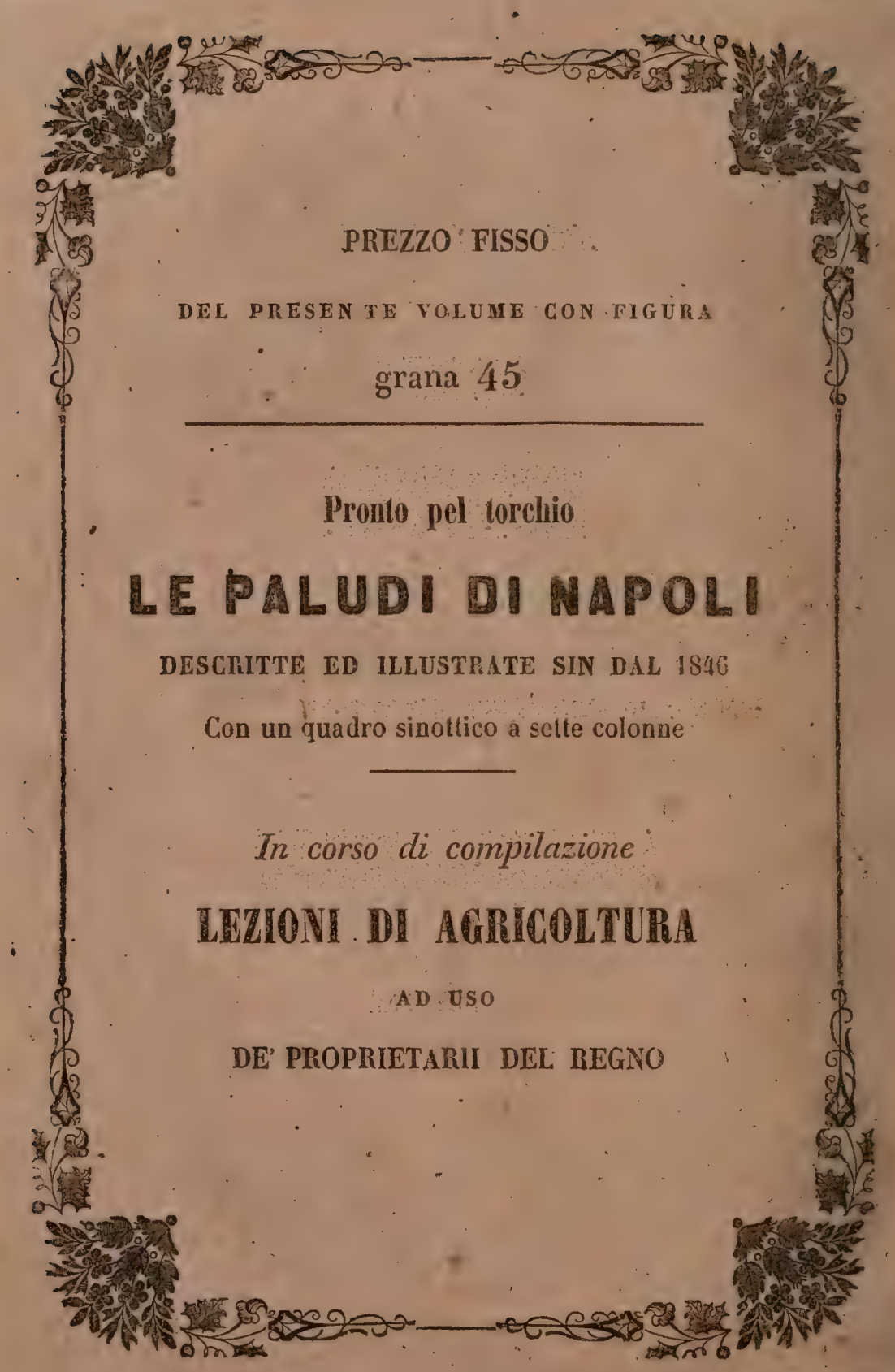







\section{QK 332 . B 78 York Botanical Garden Library}

Bruni, Achille/Descrizione botanica gen III' A 


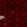

